\title{
New species and records of Stenus (Nestus) of the canaliculatus group, with the erection of a new species group (Insecta: Coleoptera: Staphylinidae: Steninae)
}

\author{
Alexandr B. RYVKIN \\ Laboratory of Soil Zoology \& General Entomology, Severtsov Institute of Problems of Ecology \& \\ Evolution, Russian Academy of Sciences, Leninskiy Prospect, 33, Moscow, 119071 Russia. \\ Bureinskiy Nature Reserve, Zelyonaya 3, Chegdomyn, Khabarovsk Territory, 682030 Russia. \\ Leninskiy Prospekt, 79, 15, Moscow, 119261 Russia. \\ Email: vr.staph@gmail.com
}

\begin{abstract}
The canaliculatus species group of Stenus (Nestus) is redefined. Four new Palaearctic species of the group are described and illustrated: $S$. (N.) alopex sp. nov. from the Putorana Highland and Taymyr Peninsula, Russia; $S$. (N.) canalis sp. nov. from SE Siberia and the Russian Far East; $S$. (N.) canosus sp. nov. from the Narat Mt Ridge, Chinese Tien Shan; $S$. (N.) delitor sp. nov. from $\mathrm{C} \&$ SE Siberia. New distributional data as well as brief analyses of old records for fourteen species described earlier are provided from both Palaearctic and Nearctic material. S. (N.) milleporus Casey, 1884 (= sectilifer Casey, 1884) is revalidated as a species propria. S. (N.) sphaerops Casey, 1884 is redescribed; its aedeagus is figured for the first time; the aedeagus of $S$. (N.) caseyi Puthz, 1972 as well as aedeagi of eight previously described Palaearctic species are illustrated anew. A key for the identification of all the known Palaearctic species of the group is given. A morphology and ecology based analysis of the main evolutionary trends within the group is provided. A lectotype is designated for $S$. (N.) melanopus Marsham, 1802; its Siberian and NE European records are supposed to be erroneous; the monotypic melanopus species group is erected.
\end{abstract}

Key words. Holarctic, Palaearctic, Nearctic, fauna, bionomics.

Ryvkin A.B. 2012. New species and records of Stenus (Nestus) of the canaliculatus group, with the erection of a new species group (Insecta: Coleoptera: Staphylinidae: Steninae). European Journal of Taxonomy 13: 1-62. http://dx.doi.org/10.5852/ejt.2012.13

\section{Introduction}

The canaliculatus group was erected by L. Benick (1925) in his keys for Palaearctic Stenus species groups. In the short preface to the named article, Benick indicated that the keys provided by him were meant for both practical identification and reflecting phylogenetic relationships between different species. It is clear that a harmonious combination of these two principles is an unrealizable ideal, especially in the case of Steninae, which are a group with extensive parallelism in many lineages. In addition, this circumstance makes all attempts to apply formal cladistics in morphology-based phylogenetic studies of the subfamily ineffective. Benick's definitions of the species groups could not avoid the 
problems connected with the form of the key in his article. The canaliculatus group within the subgenus Nestus Rey, 1884 was defined by the following characters (in the translation below I try to reformulate most hazy items): 1) abdominal segments [3 to 7] distinctly uninterruptedly margined on each side; 2) [anterior visible] abdominal tergites with four short longitudinal basal keels each; 3) pubescence not greyish-silvery; 4) maxillar palpi with basal segment yellow; 5) head not broader than elytra between humeri; 6) body with ground-sculpture developed, otherwise head unusually narrow; 7) body larger; legs usually dark; 8) median longitudinal furrow of pronotum complete, rarely shortened. One can see that the characters have been of unequal diagnostic value. The structure of abdominal tergites as well as their lateral margination and even coloration of maxillar palpi may be regarded as features at the species group level, whereas the body size, colour of legs, proportions of the forebody parts, etc. are fit for the discrimination of particular species only. The character of puncturation, macro- and microsculpture vary widely within each of the species groups in Nestus. The long and sharp median longitudinal furrow of the pronotum seems to be a detail typical of any species of the group being discussed; but a similar feature has also arisen independently in other complexes (e.g. in S. (N.) melanopus (Marsham, 1802), see below), and careful phylogenetic analysis is necessary in every case to prove a real relationship; on the other hand, the furrow may be very feeble and vague in some species of the canaliculatus group (S. (N.) raddei Ryvkin, 1987, some samples of $S$. (N.) canaliculatus Gyllenhal, 1827 and $S$. (N.) nitens Stephens, 1833 , etc). It is for the reasons aforementioned that a species group definition based on external morphological criteria solely or primarily has proved to be unsatisfactory. Fortunately, the male genitalia of Steninae, being extremely multiform at the species level, can nevertheless be used in their phylogenetically conservative general structure to establish and confirm the natural groups. Therefore, I have used these characters in the new diagnosis of the canaliculatus group (see below). I do not consider here the synonymization of the subgenus Nestus Rey, 1884 with Stenus s.str. undertaken by Puthz (2001), as the matter is discussed by me in a separate paper (Ryvkin 2011).

Since a key for identification with brief distributional characteristics was given by me for continental Palaearctic species of the canaliculatus group (Ryvkin 1987), some new taxa have been described (Puthz 1987, 2006), and vast material of the group, from both the Palaearctic and the Nearctic, became available to me for study. The material from the former USSR and China is of particular interest since both countries together cover the greater part of the Palaearctic region and are still the least investigated in the faunal aspect. Steninae of the USA and Canada seem also to be studied insufficiently: numerous papers of Puthz (see the list of references below) dealt only with part of the species and with part of the territory; the Nearctic material of many American museums looks rather scanty in comparison with their Neotropical collections. The list of staphylinid beetles provided by Campbell \& Davies (1991) for Canada \& Alaska contains no references to material and sources published; as a result, it is impossible to verify the data cited, and many erroneous data of old authors remain in the list concurrently with recent records. Unfortunately, the same problem arises with the Palaearctic Catalogue by Smetana (2004) which, owing to the absence of references to the sources of information, turned into a simple check-list, untestable and unusable, in spite of the herculean labour undertaken by the author.

The annotated list given below contains the results of processing the material on the canaliculatus group, including the descriptions of four new species as well as numerous new data on distribution with brief analyses of old records for fourteen representatives previously described from both the Palaearctic and the Nearctic (other species, for which no new data have been provided, are listed in the 'Taxa included' section only, each marked with an asterisk and supplied with short distributional data and a reference to the source). A key for the identification of all the known Palaearctic species of the group is given. 


\title{
Material and methods
}

The original material, which the present paper is based on, is represented by 591 specimens (288 $\widehat{\partial}$, 303 웅). A large proportion of these has been captured during my numerous field trips in 1976-2009; others have been either donated or loaned to me for study by many colleagues and institutions. The names of the respective collections in which the material is deposited now are abbreviated and listed below.

In the annotated list below, both type and unique specimen labels are cited in single quotes completely (the type and historic labels with vertical strokes to separate different lines of a label); square brackets are used to complete label data; if necessary, the labels are supplied with remarks in angled brackets; the labels of other specimens are given in English without indicating the original language. The material identified questionably is shown with a question-mark in angled brackets. Catalogue references are given only for the sources concerning essential aspects of taxonomy or distribution; for other references see Herman (2001). Catalogues and compilations have been used mainly if they include any original data and/or interpretations (J. Sahlberg 1899, 1900; Jakobson 1909; Bernhauer \& Schubert 1911; Tichomirova 1973, Campbell \& Davies 1991, Ryabukhin 1999), while others (Heyden 1881, 1898, etc) are mentioned, as needed, either ad notam or for correcting appreciable errors. The numerous latest regional check-lists, where sources of individual records have been concealed, are mostly not cited below. In cases of doubtful citing, the question-mark in angled brackets was placed either behind the species name (for faunistic records) or before it (for doubtful synonymy) in the respective lines of catalogue sections. If a quite evident misidentification has not been proved by a revision of the material till now, this fact is indicated in the respective catalogue record as '?!' in angled brackets behind the species name. In all cases, when the material identified before by other authors is used, the references to their identifications are given.

The line drawings were made with the drawing apparatus RA-7 (LOMO); the photomicrographs were produced with a Webbers MYscope 130-M digital microscope camera (1.5 Mpix); the images were processed using Helicon Focus 3.20.3 Free software (www.helicon.com.ua).

The measured proportions of body parts are given in points of an eyepiece linear micrometer in a binocular microscope at 56x magnification; prementum of some Stenus species was measured dorsally from its base to the base of the glossae, in glycerine preparations; the head length is measured along the midline from the neck restriction to the base of the labrum.

\author{
Abbreviations

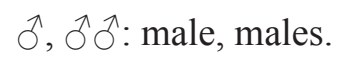 \\ ㅇ, 우우: female, females. \\ ex: specimen, specimens. \\ HT: holotype. \\ PT: paratype.
}

AR: Collection of A.B. Ryvkin, Moscow, Russia.

ASh: Collection of A.V. Shavrin, Daugavpils, Latvia.

AVS: Collection of A.V. Sokolov, Moscow, Russia.

AYuS: Collection of A.Yu. Solodovnikov, Copenhagen, Denmark.

BM: Natural History Museum (=British Museum (Natural History)), London, UK (M. Barclay, M. Brendell).

DUL: Systematic Biological Institute, Daugavpils University, Latvia (A.V. Shavrin).

FMNH: Field Museum of Natural History, Chicago, USA (M. Thayer, A. Newton).

GMR: Collection of G.M. de Rougemont, London, UK. 
IBPM: Institute of Biological Problems of the North, Magadan, Russia (D.I. Berman, E.G. Matis, A.S. Ryabukhin).

IG: Collection of I.N. Goreslavets, Samara, Russia.

IRSN: Institut Royal des Sciences Naturelles de Belgique, Bruxelles (D. Drugmand, M. Peeters)

KG: Collection of K.A. Grebennikov, St. Petersburg, Russia.

LH: Collection of L. Hromádka, Praha, Czech Republic.

MHNG: Muséum d'Histoire Naturelle, Genève, Suisse (G. Cuccodoro).

MTD: Museum für Tierkunde, Dresden, Germany (K.-D. Klass).

ONK: Collection of O.N. Kabakov, St. Petersburg, Russia.

ShIN: Collection of Sh.-I. Naomi, Chiba, Japan.

SSTC: Smolensk State Teacher's College, Smolensk, Russia (M.Yu. Gildenkov).

TI: Collection of Tateo Ito, Kyoto, Japan.

UASM: E.H. Strickland Entomological Museum, University of Alberta, Edmonton, Canada (D. Shpeley).

VP: Collection of V. Puthz, Schlitz, Germany.

VS: Collection of V.B. Semenov, Moscow, Russia.

ZIN: Zoological Institute, Russian Academy of Sciences, St. Petersburg, Russia (†G.S. Medvedev).

ZMH: Zoological Museum Helsinki, Finland (H. Silfverberg).

ZMMU: Zoological Museum of Moscow State University, Moscow, Russia (A.A. Gusakov).

ZMRU: Zoological Museum of Rostov State University, Rostov-on-Don, Russia (E.A. Khachikov).

\section{Results: systematic part}

\section{Redefinition of the canaliculatus group}

\section{Diagnosis}

Body size moderate; body length of the known species: 2.8 to $4.7 \mathrm{~mm}$.

Head fairly small to fairly broad in comparison to elytra and pronotum. Upper surface between eyes feebly evenly convex, nearly flat, to slightly concave as a whole; with longitudinal elevations and impressions nearly absent to well developed. Antennae rather short to long, with the club segments more or less elongated. Internal tooth of each mandible placed a little before the middle, directed only slightly dorsally of the main plane. Maxillar palpi with yellow and not shortened basal segment. A considerable reduction of the adhesion capture apparatus may be supposed for all the members of the group (for more details see the Remarks section below).

Pronotum with long and sharp longitudinal median furrow more or less deep, less often very feeble, stroke-shaped.

Elytra nearly rectangular, large, with short rounded humeri, to shortened, slightly trapezoid with humeri less developed.

Legs moderately long; the segment 4 of tarsi without emargination; segment 1 of metatarsi about the same length as to a bit longer than segment 5 but distinctly shorter than segments 2 to 4 together.

Abdomen moderately convex, with paratergites evident on abdominal segments 3 to 7 ; four anterior visible tergites each with four short but evident longitudinal keels at basal part; posterior margin of the tergite 7 with well-developed fine membranous fringe.

Puncturation of pronotum and elytra diverse, but always non-rugose. 
Ground sculpture cellular or net-shaped to entirely absent.

Pubescence moderate, contiguous to fairly long, outstanding.

MALE. Legs without specific features; posterior margin of abdominal sternites 6-7 shallowly emarginated to about straight; abdominal sternite 8 with a broad but not deep, rounded or angularly rounded emargination of posterior margin; abdominal sternite 9 with large posterolateral teeth not incurved inwards; ventral depressions of sternites 6-8, if present, flat and feeble, never flanked by sharp keels. Aedeagus relatively large; median lobe archetypically lanceolate, with apical sclerotized part to a variable extent angular to angularly rounded; endophallus with paired medial bands (optionally, an unpaired bar may also be distinct), small but visible lateral parts, and $\mathrm{H}$-shaped expulsion clasp producing lateral portions fairly broad; basal tube of diverse structure, more or less sclerotized. Parameres cylindrical, with apical broadening, if present, very feeble, rather vague; apico-internal setiferous surface differentiated poorly to moderately, setae fairly uniform.

Female. Posterior margin of abdominal sternite 8 broadly rounded to angularly rounded; each valvifer with posterolateral tooth directed backwards. Spermatheca sclerotized to a greater or lesser extent.

In the structure of abdomen, the male genitalia, the abdominal sternite 9 of both males and females (valvifera), the group under consideration is most closely related to the boops and palposus groups of the same subgenus Nestus. It differs from these by the structure of the mandibles, by the adhesion capture apparatus more or less shortened, by the pronotum with long and sharp, line-shaped, longitudinal median furrow, by the evidently narrower aedeagus, by the endophallus with the expulsion clasp's transversal bridge adjoining to the lateral pieces near their middle, by the abdominal sternite 9 with posterolateral teeth not being curved inwards, by the male legs without peculiar features (not all the species of the named groups have such features well developed); from the palposus group it can be easily distinguished by the yellow basal segment of the maxillar palpi. The differences from $S$. $(N$.) melanopus Marsham, 1802, similar externally to some species of the canaliculatus group, are given in the diagnosis of the melanopus group and in the key (see below). In many characters of the structure of abdomen and male genitalia, the canaliculatus group also resembles the aggregate including the large and diverse atratulus-fuscipes-cautus-crassus complex and some isolated American members, as S. (N.) mendosus Puthz, 1971 (1971d) and S. (N.) sordidus Puthz, 1988, but phylogenetic relations within the named aggregate remain poorly understood; therefore a thorough analysis seems to be premature. In external morphology, the canaliculatus group can easily be distinguished from all the species of the mentioned complex by the presence of the long and sharp, line-shaped, longitudinal median furrow of the pronotum.

\section{Taxa included}

S. (N.) alopex sp. nov., S. (N.) brivioi Puthz, 1972, S. (N.) canaliculatus Gyllenhal, S. (N.) canalis sp. nov., 1827, S. (N.) canosus sp. nov., S. (N.) caseyi Puthz, 1972, S. (N.) confusus J. Sahlberg, 1876, S. (N.) delitor sp. nov., S. (N.) dolosus Casey, 1884, S. (N.) geminorum Puthz, 1973* (USA: Washington; Canada: BC, see Puthz 1973b: 208), S. (N.) idoneus Sharp, 1887* (Mexico, see Sharp 1887: 799; Puthz 1968: $11<$ as laniger Puthz, 1968>), S. (N.) illotulus Puthz, 1972, S. (N.) illusor Ryvkin, 1987, S. (N.) immigratus Puthz, 2006* (China: Taiwan: Ilan Hsien: Shen Mi Lake, 1100 m, see Puthz, 2006: 187), S. (N.) labilis Erichson, 1840, S. (N.) latipennis J. Sahlberg, 1880, S. (N.) milleporus Casey, 1884, S. (N.) nitens Stephens, 1833, S. (N.) raddei Ryvkin, 1987, S. (N.) shogun Puthz, 1987* (Japan: Nishigo Uzan, see Puthz 1987: 45), S. (N.) sphaerops Casey, 1884, S. (N.) vinnulus Casey, 1884. 


\section{Notes on comparative morphology and evolution}

As most other polytypic groups in Steninae, the canaliculatus group reveals diverse evolutionary trends quite evident at a comparison between species of the same lineage. A concise review of the mentioned trends is given below.

A. Upper surface of head between eyes tends either to become flattened, at most slightly convex (S. shogun, S. nitens, S. illotulus, S. raddei, S. caseyi, S. canaliculatus, S. canalis, S. canosus, S. alopex) or to produce distinct longitudinal impressions with median elevation in between ( $S$. labilis, S. idoneus, S. vinnulus, S. brivioi, S. confusus, S. delitor, S. illusor, S. latipennis, S. immigratus, S. geminorum, S. sphaerops, S. dolosus, S. milleporus). The latter tendency seems to arise and develop independently, at least within the complexes labilis-confusus-delitor-illusor-latipennis-sphaerops and dolosus-milleporus, in spite of the fact that the latter is closely related in the shape of the male genitalia to the 'canaliculatus s.str.' complex characterised by a flat front.

B. For three species studied by him (S. canaliculatus, S. nitens, S. labilis), Betz (1996) has shown a considerable reduction of the adhesion capture apparatus. It is expressed both in the degeneration of sticky cushions of paraglossae (decreasing of their surface and number of adhesive setae etc.) and in a shortening of the labium. No data on labium length have been provided by the named author but, as it follows from the text, $S$. milleporus (as sectilifer Casey, 1884), S. caseyi, S. vinnulus, as well as S. melanopus, which is regarded as a member of the same group, have also been studied in this respect.

I have measured the relative length of the prementum (eulabium after Weinreich 1968) for six species of the canaliculatus group: S. canaliculatus (I: 17, 0.53; II: 16, 0.53), S. nitens (33, 0.87), S. labilis (37, 1.12), S. illotulus (26, 0.84), S. raddei $(35,1.09)$, S. latipennis $(31,0.79)$; and for three species of other groups of the subgenus Nestus Rey, 1884: S. ruralis Erichson, $1840(47,1.52)$ from the palposus group, S. argus Gravenhorst, 1806 (47, 1.74) from the fuscipes group, and S. melanopus Marsham, $1802(41,1.46)$ presumably representing a monotypic group. In each case above, the first number in brackets means length of prementum whereas the second one is a rounded ratio between the former and the length of the head (two specimens have been measured for $S$. canaliculatus). One can see that the data split the examined species into three clusters: a) S. canaliculatus; b) the remaining species of the canaliculatus group; c) the species of other groups; both total and relative length of prementum increases from category a) to c). It can be supposed that the reduction of the eulabium is a characteristic feature of all the members of the canaliculatus group, but it must be verified by a specific study. The shortening of the adhesion capture apparatus seems to correlate with some other modifications of the mouth parts, namely the more proximal position of the internal tooth of mandibles as well as its lesser deflection off the main plane of the mandible.

The small median notch of the labrum is a specific character of $S$. canaliculatus and the most closely related Palaearctic species (S. canalis, $S$. canosus, $S$. alopex). Though the functional explanation of this feature is obscure as yet, one can suppose it to correlate with the extreme reduction of the adhesion capture apparatus in this species complex. The Nearctic members of the same complex (S. milleporus and $S$. dolosus) have only a broad and shallow emargination on the anterior margin of the labrum; the margin is slightly sinuate at the middle in $S$. nitens, $S$. illotulus, S. raddei, S. caseyi, S. brivioi, $S$. vinnulus; lastly, the species of the labilis-confusus-delitor-illusor-latipennis-sphaerops line have the margin nearly straight.

Betz (1996) supposed the reduction of the adhesion capture apparatus in the canaliculatus group to be a result of miniaturisation, but this explanation does not stand up to any criticism. Indeed, the smallest specimens of the species within the group may be considered among the medium-sized representatives of the genus, while even much smaller members of both the pusillus and the pumilio groups have the adhesion capture apparatus well developed ( $S$. (N.) pusillus Stephens, 1833 has been studied by Betz (1996) himself). 
I believe that the modification of the mouth parts should be connected more likely to a particular suite of potential prey species. Unfortunately, Betz used only springtails and (to a lesser extent) winged aphids in his interesting prey-capture experiments, but those may not exhaust the list of possible prey objects for Steninae. I did not experiment with species of the canaliculatus group; however other Stenus species were capturing nymphs of Miridae (Insecta: Hemiptera: Heteroptera) and Cicadellidae (Insecta: Hemiptera: Auchenorrhyncha), juvenile spiders of the family Linyphiidae (Arachnida: Aranei), small mites (Arachnida: Acari), small pyralid caterpillars (Insecta: Lepidoptera: Pyralidae), small flies (Diptera), soil Enchytraeidae (Oligochaeta), in addition to the aforementioned objects, in my unpublished experiments of 1985-1986. I consider it necessary to undertake a more detailed study of prey for the species of the group being discussed.

C. The tendency of legs to become longer has most likely taken place independently in two species complexes: delitor-illusor-latipennis-confusus-sphaerops and nitens-illotulus-raddei (only the species with the $1^{\text {st }}$ segment of the metatarsus longer than the $5^{\text {th }}$ are listed). S. canaliculatus, S. canalis, S. canosus, $S$. alopex, $S$. milleporus, $S$. caseyi have metatarsi with the $1^{\text {st }}$ segment about as long as $5^{\text {th }}$; in S. dolosus, S. brivioi, S. vinnulus, and S. labilis, metatarsi remain short (with the $1^{\text {st }}$ segment evidently shorter than the $5^{\text {th }}$ ).

D. The plesiomorphic aedeagus with a lanceolate median lobe and unmodified cylindrical parameres (as in S. labilis) can be transformed by different ways. The distal sclerotized part of the median lobe may be shortened, either angularly (as in S. sphaerops) or rounded-angularly (in the delitor-illusor-latipennis line); in only one case with a small apical denticle (in $S$. vinnulus); this modification usually correlates to a lengthening of the whole aedeagus (see Figs. 6, 7A-I) or only the median lobe (as in S. confusus, see Fig. 2E), although this correlation has not been observed in $S$. raddei, belonging to another lineage (Fig. 2A, B). The tendency of the median lobe to become narrower (as in S. shogun, S. brivioi, S. alopex) is another way of transformation, being peculiar to the lineages of nitens and 'canaliculatus s.str.'; one can suppose that the aedeagus of Mexican S. idoneus has evolved in this way. All the modifications mentioned may be followed by forming a more or less developed medial impression or/and keel at the distal sclerotized part of the median lobe.

The main trend in the evolution of the parameres is a differentiation of their setiferous surface. It is expressed more frequently in a flattening of the apicointernal side of each paramere or, on the contrary, in forming a one-sided swell which setae are attached to.

Based on all the characters and tendencies briefly discussed above, we can subdivide the canaliculatus group into four subgroups: the monotypic labilis subgroup ( $S$. labilis), the nitens subgroup (S. immigratus, S. nitens, S. raddei, S. illotulus, S. shogun, S. caseyi, S. geminorum, S. brivioi, S. idoneus), the canaliculatus s.str. subgroup ( $S$. dolosus, S. milleporus, S. canaliculatus, S. canalis, S. canosus, S. alopex), and the confusus subgroup (S. confusus, S. delitor, S. illusor, S. latipennis, S. sphaerops, S. vinnulus).

\section{Bionomics}

The majority of the species for which enough data have been presented can be grouped into swamp inhabitants and alluvial ones. The former category, comprising the species dwelling in moss and litter of eutrophic and mesotrophic wet habitats, definitely includes $S$. nitens, $S$. raddei, S. illotulus, S. caseyi, S. brivioi and S. sphaerops(?), whereas the latter category consists of the members inhabiting open alluvia, like shingle beds, sandy, clayey or silty banks, sometimes sparsely overgrown with grass but without any more or less evident peat-moss or litter layer: S. labilis, S. delitor, S. latipennis, S. confusus, S. dolosus, S. canaliculatus, S. canosus, S. canalis. There are also some species (S. alopex, S. vinnulus, and supposedly $S$. illusor) living in biotopes of both categories. The peculiar swamp species, excluding 
S. sphaerops for which too little data are available, are represented only by the nitens subgroup, which is supposed to be closest to the common ancestor of the canaliculatus group.

\section{General distribution}

Palaearctic except subtropical regions (spotty-relict in the southernmost temperate territories); the whole Nearctic; the Caribbean Islands. Many species of the group are represented in the northernmost territories of the Holarctic.Thus, five of the fifteen Palaearctic species have been recorded for the Taimyr Peninsula and Putorana Highland, which is more than 1/4 of the regional stenine fauna.

\section{Species list}

Stenus (Nestus) labilis Erichson, 1840

(Fig. 1E)

Stenus labilis Erichson, 1840: 697.

Stenus labilis - Thomson 1857: 223. - J. Sahlberg 1871: 415. — J. Sahlberg 1900: 29. — L. Benick 1921a: 146. — Puthz 1971b: 34. — Silfverberg 1988: $21<$ types of lapponicus J. Sahlberg, 1876>. Sokolov 2003: 1272. — Shavrin \& Puthz 2007: 122.

Stenus labilis <?!> - Fauvel 1865: 306. - Iljin 1926: 225.

Stenus (Nestus) labilis - Poppius 1909b: 19. — Jakobson 1909: 481. — Renkonen 1935: 29. — Palm 1961: 90, 98. — Puthz 1967a: 49. — Puthz1967d: 293. — Tichomirova 1973: 173. — Shilov 1975: 58.

— Ryvkin 1987: 159.

Stenus (Nestus) labilis <?!> - Poppius 1909a: 17.

Stenus (s. str.) labilis - Campbell \& Davies 1991: 111.

Stenus canaliculatus - C. Sahlberg 1832: 428.

Stenus lapponicus J. Sahlberg, 1876: 56.

Stenus lapponicus - J. Sahlberg 1880: 78. — Heyden 1881: 78. — J. Sahlberg 1899: 340.

Stenus (Nestus) lapponicus - Jakobson 1909: 481. — Hansen et al. 1939: 32 (pars).

Stenus latipennis - Renkonen 1941: 105.

\section{Material examined}

FINLAND: $1 \hat{\jmath}$, 'Lac. Kemi' <printed on yellowed white rectangle>, 'J. Sahlb.[erg]' <printed on yellowed white rectangle $>$, '77' $<$ hand-written with Indian ink on grey square $>$, 'St. labilis Er. typ (=lapponicus J. Sahlb.) <black Indian ink $>$ J. Sahlberg det. $<$ printed in italics $>$ ' $<$ common determinative label by J. Sahlberg> (ZIN); 1 + , 'Turtola' <printed on yellowed white rectangle>, 'J. Sahlb.[erg]' $<$ printed on yellowed white rectangle>, 'Stenus lapponicus' $<$ black Indian ink $>$ (ZIN); 1 Oे, 'Tenojoki' $<$ printed on yellowed white rectangle>, 'J. Sahlb.[erg]' <printed on yellowed white rectangle>, 'St. labilis Er. (=lapponicus J. Sahlb.)' <black Indian ink>, 'c.[ollection] of A. Jakovlev'<printed label in Russian> (ZIN).

RUSSIA: 1 , Arkhangelsk Area, $10 \mathrm{~km} \mathrm{~N}$ of Naryan-Mar, near Iskateley, sandy bank of Pechora River, 14 Jul. 1988, P.K. Yeryomin leg. (AR); 1 , Arkhangelsk Area, 5 km S of Naryan-Mar, bank of right confluent of Pechora River, on silt, 19 Jul. 1988, P.K. Yeryomin leg. (AR); 1 q, Arkhangelsk Area, NaryanMar, swamp, 29 Jul. 1988, P.K. Yeryomin leg. (AR); 1 ðै, Arkhangelsk Area, Nenetzkiy Autonomous Region, Pechora River delta, sedge meadow at lake shore, 7 Sep. 1998, N.S. Mazura leg. (AR); 1 , 'Yugorskiy Shar, near Velikaya River, tundra, 27.viii.1921, E. Abakumova leg.' <in Russian>, 'labilis Er. L. Benick det.' (ZIN); 1 गे, Arkhangelsk Area, near Amderma, flood-plain of Amderminka River, 


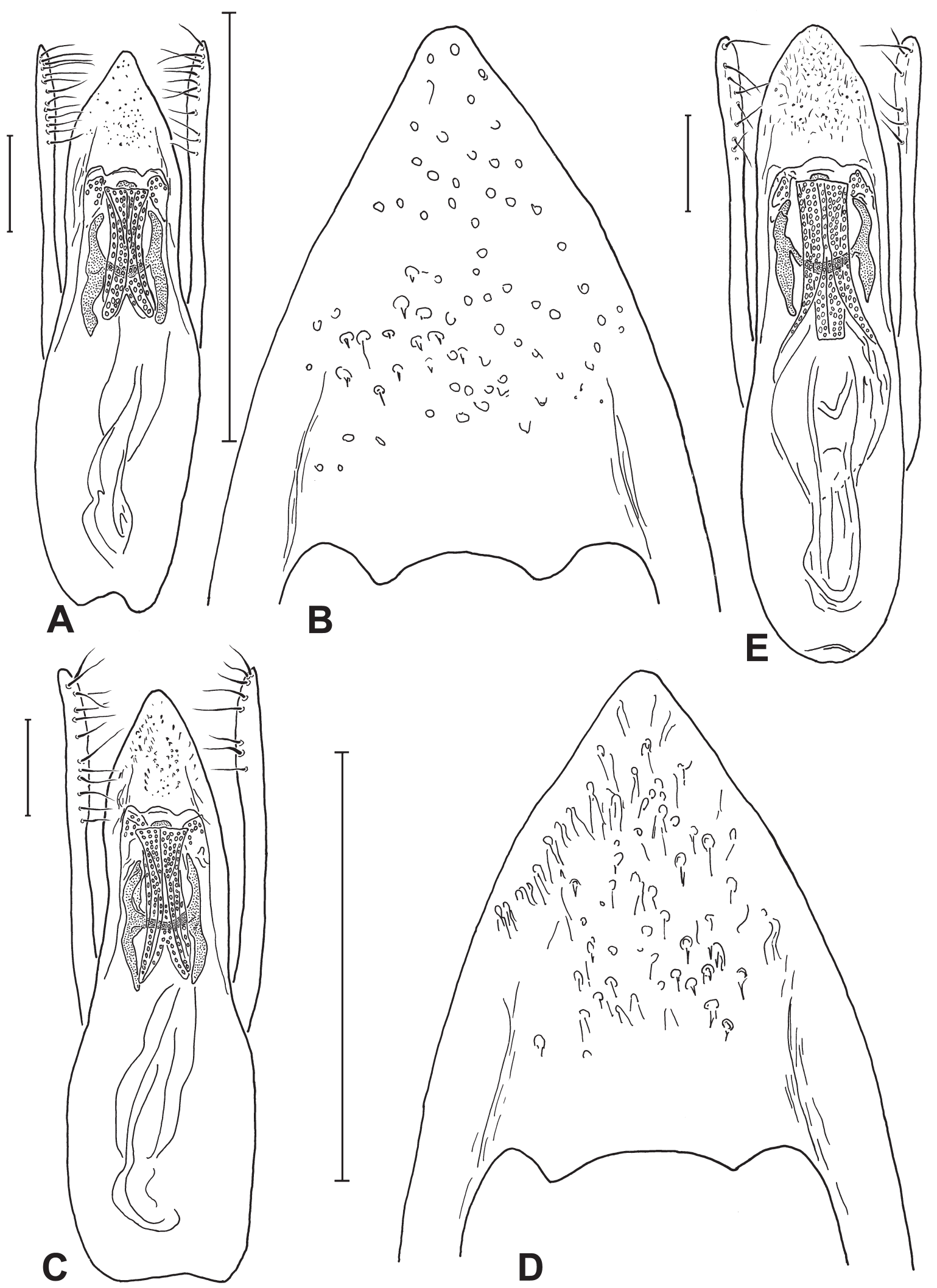

Fig. 1. - A-D: Stenus (Nestus) nitens Stephens, 1833: ô genitalia, ventral view. A-B: (Russia: Moscow Area: Gorenki). C-D: (Russia: Evenkia: Stolbovaya River). A, C. Aedeagus. B, D. Apical part of median lobe. - E. S. (N.) labilis Erichson, 1840: §̂̉ (Russia: S Yamal: nr. Salekhard). Aedeagus, ventral view. Scales $=0.1 \mathrm{~mm}$. 
on silt, 9 Jul. 1988, P.K. Yeryomin leg. <the specimen destroyed by dermestids, remained on cotton> (AR); $1 \hat{\jmath}$, South of Yamal Peninsula, 110 th $\mathrm{km}$ by railway $\mathrm{N}$ off Obskaya station, puddle, 6 Aug. 2001, P. Petrov leg. (AR); 1 J, W Siberia, Salekhard, Poluy River bank, 27 Jun. 1954, I. Telishev leg. <det. V. Puthz, 1971> (ZMMU); 1 đ̂, Yamalo-Nenetskiy Autonomous Region, nr. Salekhard, 5 Jul. 1940 (AR); 1 q, Muzhi-na-Obi, 23 Jun. 1952, I. Telishev leg. (ZMMU); 1§, Beryozov, 13 Jul. 1932, Telishev leg. <det. V. Puthz, 1971, with earlier determinative label by unknown author: 'Nestus melanarius Steph.'>

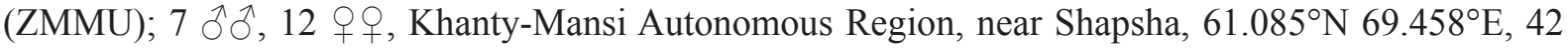
m a.s.1., 1-4 Aug. 2010, K. Tomkovich leg. (AR, ShIN); 1 , Khanty-Mansi Autonomous Region, Surgutskiy District, Surgutskiy Refuge, Ob' River basin, Materikovyi Pasl Channel, bank of rill flowing into channel: near water (clay), Carex sp., Poaceae gen. sp., Veronica ? longifolia, Asteraceae, small

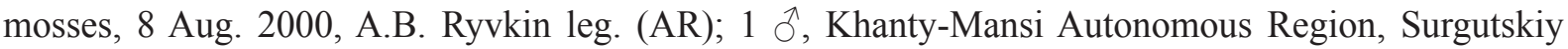
District, Yuganskiy Nature Reserve, Malyi Yugan River, near cordon below Lyarykni River mouth, on sand and clay and in grass litter among Carex spp. at river bank, 15 Aug. 2003, A.B. Ryvkin leg. (AR); $1 \hat{0}, 1$ क, Tyumen Area, Uvatskiy District, $10 \mathrm{~km} \mathrm{~S}$ of Gornoslinkino, near Tobolsk Field Research Station of Severtsov Institute, among Carex spp. on flood-plain of Varpak River, 25 Sep. 2003,

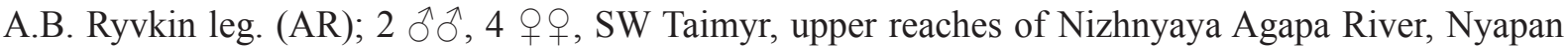
Ridge, $70^{\circ} 04^{\prime} \mathrm{N} 87^{\circ} 36^{\prime} \mathrm{E}$, detritus at shore of Ladannakh Lake, 17 Jul. 2001, A.V. Sokolov leg. (AVS); 1 , same locality and collector, shingle bed, 2 Jul. 2001 (AVS); 2 đô, 3 q $q$, same locality, soil traps on swamp, 8 Jul.-15 Aug. 1999, O. Makarova leg. (AVS); 1 đ, 1 q, Taymyr, Tammot Lake, $10 \mathrm{~km}$ ENE of Lukunskoye cordon, on silt under flood-plain, Salix sp., 15 Jul. 1989, P.K. Yeryomin leg. (AR); 1 A, same locality, slightly silty sandy lake shore with small shingles, 24 Jul. 1989, P.K. Yeryomin leg. (AR); 1 , , Taymyr, island on Khatanga River, $10 \mathrm{~km} \mathrm{NE}$ of Oboynaya Village, slightly silty sandy river bank, 19 Jul. 1989, P.K. Yeryomin leg. (AR); 1 Oे, Taymyr, Maymecha River mouth, Yantardakh Mount, 7 Jul. 1971 A.P. Rasnitsyn, A.G. Ponomarenko, I.D. Sukatcheva, V.V. Zherikhin leg. (AR); 1 ô, 1 q, Taymyr, Khatangskiy District, Taymyr Nature Reserve, Ary-Mas: right bank of Novaya River, lake ESE of Ulakhan-Yuryakh River mouth, mosses, litter and sweeping at lake shore: Carex spp., Comarum palustre etc., 20 Jul. 1992, A.B. Ryvkin leg. (AR); 1 ô, Taymyr, Khatangskiy District, Taymyr Nature Reserve, Ary-Mas: Novaya River basin, Bogatyr'-Yuryakh River near mouth, steep river bank and high river plain, clay, drift, Poaceae gen. sp., Carex spp., Salix spp., Alnus fruticosa, Ribes sp., 21 Jul. 1992, A.B. Ryvkin leg. (AR); $5 \hat{\jmath} \hat{\jmath}$, Taymyr, Khatangskiy District, Taymyrskiy Nature Reserve, Ary-Mas: left bank of Novaya River near field research station, river bank, sand with thin layer of silt and drift near water, 22 Jul. 1992, A.B. Ryvkin leg. (AR).

CANADA: 2 우 'NWT, Loc. 06-77, Anderson River delta, Boat Island, Salix alexensis zone: traps, 26 Jun.-15 Jul 1977, D. Shpeley, G.E. Ball leg. (Anderson River Exp. - 1977)’ (UASM).

\section{Remarks}

This species was named by Erichson (1840) from 'Fennia', having been misidentified earlier by C. Sahlberg (1832) as S. canaliculatus Gyllenhal, 1827; redescribed as S. lapponicus J. Sahlberg, 1876 from Finland (up to $68^{\circ} 30^{\prime} \mathrm{N}$ ), but cited as labilis (only!) by J. Sahlberg (1900) in his catalogue of the beetles of the fauna of Finland. Only the Norwegian, Swedish, Finnish, and N Russian records (Thomson 1857; J. Sahlberg 1871, 1899, 1900; Poppius 1909b; Jakobson 1909; Palm 1961; Puthz 1971b) seem to be reliable for the European range of the species. The records for France/Italy (Fauvel 1865), as well as for the Yekaterinoslav Government (=Dnepropetrovsk Area) of the Ukraine (Iljin 1926), are to be regarded as results of misidentification. In Western and Central Siberia, S. labilis has been known from Polar Ural to W Taymyr (J. Sahlberg 1880, L. Benick 1921a, Puthz 1967a, Shilov 1975, Sokolov $2003<$ the latter is based on my identifications $>$ ). Puthz (Shavrin \& Puthz 2007), without new material, considers the species to be distributed in E Siberia, but the only record by Poppius (1909a), for the Lena basin ('Mehrere Exemplare auf feuchten, Moos-bewachsenen Wiesen auf der Insel Agrafena, 1.VIII!.'), has been confirmed by neither subsequent authors nor my vast material from this spacious territory. 
Thus, $S$. labilis is unlikely to be represented in Siberia eastwards of E Taymyr. The new material above confirms the distribution of the species in N America (Campbell \& Davies 1991: Alaska, Yukon Territory, Northwest Territories). Most captures known to me are confined to alluvial habitats.

Stenus (Nestus) nitens Stephens, 1833

(Fig. 1A-D)

Stenus nitens Stephens, 1833: 300.

Stenus nitens - Waterhouse 1858: 28. — J. Sahlberg 1876: 58. — Poppius 1899: 39. — J. Sahlberg 1899: 340. — J. Sahlberg 1900: 29. — Szujecki 1960: 297. — Puthz 1971b: 34. —Tichomirova 1982: 213. — Pisanenko \& Puthz 1991: 170. — Puthz \& Zanetti 1995: 18. — Shavrin \& Puthz 2007: 125.

Stenus (Nestus) nitens - Jakobson 1909: 481. — Reitter 1909: 158. — Renkonen 1935: 29. — Szujecki 1961: 34. — Palm 1961: 90. — Puthz 1967d: 293. — Puthz 1971a: 82. — Tichomirova 1973: 173. — Shilov 1975: 58. — Ryvkin 1987: 159. — Dauphin 1993: 188. — Semenov 2004: 12.

Stenus (Nestus) nitens <?!>- Poppius 1909a: 16. — Bordoni 2004: 120.

Stenus aemulus Erichson, 1839: 541.

Stenus aemulus - Hochhuth 1872: 154 (as S. aemulus Gyllenhal).

\section{Material examined}

FINLAND: 1 ô, 'Fennia. Reitter', 'Stenus nitens Steph.', 'c.[ollection] of A. Jakovlev' < printed label in Russian $>$ (ZIN); 1 ô, 1 ㅇ, 'Fennia'<printed label on red paper>, 'J. Sahlb.[erg]' <printed on yellowed white rectangle>, 'Stenus nitens Stph.' < black Indian ink> (ZIN: ex coll. Semenov-Tian-Shanskiy); 1 ○, 'Helsingfors. 4126[=Stenus nitens Steph.] - 3' (ZIN).

RUSSIA: 1 + , 'Petropolis. 4126[=Stenus nitens Steph.] - 5', 'nitens Steph.' (ZIN); 1 +, Tyumen Area, Nefteyuganskiy District, $90 \mathrm{~km} \mathrm{~S}$ of Nefteyugansk, near Bolshoye Kayukovo Lake, geobotanical field research station of Moscow University, floating island on sedge eutrophic swamp: in hummock base, 20 Aug. 1985, R.A. Rakitov leg. (AR); 1 đ, 2 q $ᄋ$, Khanty-Mansi Autonomous Region, Surgutskiy District, Yuganskiy Nature Reserve, Nyogus'yakh River, Bisarkina cordon, moss and litter on swamp amid flood plain forest near foot of slope: Menyanthes trifoliata, Equisetum spp., Sphagnum squarrosum (sparse), Sph. ? centrale, Sph. spp., Nardosmia frigida, Carex spp., Poaceae gen. sp., Salix sp., Comarum palustre, Filipendula ulmaria, ? Tomenthypnum sp., Plagiomnium sp., etc., 27 Jul. 1998, A.B. Ryvkin leg. (AR); 1 ${ }^{\lambda}$, Khanty-Mansi Autonomous Region, Surgutskiy District, Yuganskiy Nature Reserve, Nyogus'yakh River basin near Pechpan'yakh River mouth, mosses and litter on large swamp: Carex spp., Comarum palustre, Equisetum sp., Nardosmia frigida, Eriophorum sp., Poaceae gen. spp., Rumex sp., Chamaenerion angustifolium, Salix sp., ? Aulacomnium sp., Plagiomnium sp., Ptilium cristacastrensis, Sphagnum ?russovii, Sph. sp. etc., 15 Sep. 2000, A.B. Ryvkin leg. (AR); 1 ô, 1 q, KhantyMansi Autonomous Region, Surgutskiy District, Yuganskiy Nature Reserve, Ay-Magromsy River basin, Medvezhyi Ugol cordon, mosses and litter at southern border of sedge-mossy swamp: Carex rostrata, C. spp., Comarum palustre, Equisetum spp., Sphagnum girgensohnii, Sph. spp., Plagiomnium sp., Aulacomnium sp., Calamagrostis sp., Menyanthes trifoliata, etc. among sparse young birches $\left(\mathrm{t}=+10^{\circ}-\right.$ $+5^{\circ} \mathrm{C}$ ), 4 Oct. 2002, A.B. Ryvkin leg. (AR); 1 ${ }^{\lambda}$, Khanty-Mansi Autonomous Region, Surgutskiy District, Yuganskiy Nature Reserve, Malyi Yugan River basin, 3-5 km SW of cordon at Kol-Kochen-Yagun River, mosses and litter by swampy margin at N side of lake: Carex spp., Sphagnum spp., Poaceae gen. spp., 26 Sep. 1999, A.B. Ryvkin leg. (AR); 1 q, Khanty-Mansi Autonomous Region, Surgutskiy District, Yuganskiy Nature Reserve, Malyi Yugan River, cordon below Lyarykni River mouth, open swamp with tussocks of Carex spp. and Calamagrostis sp. with Comarum palustre, Salix sp., Sphagnum squarrosum, Sph. spp., ? Aulacomnium sp. etc. among sparse young Betula sp., 17 Aug. 2003, A.B. Ryvkin leg. (AR); 
1 đ, Krasnoyarsk Territory, Turukhanskiy District, Bakhta River basin, near Keteollo Lake, Noya River source, $323 \mathrm{~m}$ a.s.l., mosses and litter among Carex spp., Filipendula ulmaria, Poaceae on tussocks and among those at open river bank, 6 Aug. 1992, A.B. Ryvkin leg. (AR); 1 đ̂, Evenkia, Baykitskiy District, Central Siberian Biosphere Reserve, Stolbovaya River 8 km up-stream of river mouth, $60 \mathrm{~m}$ a.s.l., mosses and litter on open swamp with Carex spp., Comarum palustre, sparse Menyanthes trifoliata, true mosses, Sphagnum spp. etc., 20 Sep. 1991, A.B. Ryvkin leg. (AR); 1 ô, Evenkia, Baykitskiy District, Podkamennaya Tunguska River, $175 \mathrm{~km}$ up-stream of river mouth, near Belaya Kosa Island, $55 \mathrm{~m}$ a.s.l., swamp with Comarum palustre, Sphagnum spp., Equisetum sp., Plagiomnium sp., Carex spp., etc., 23 Aug. 1990, A.B. Ryvkin leg. (AR).

\section{Remarks}

Terra typica: 'London; Suffolk'; for aemulus: 'Brandenburg'. The species is known from C \& N Europe and W Siberia (Tichomirova 1973). Hochhuth (1872) reported it (as aemulus Gyllh. <sic!>) for the Kiev Government ('Ziemlich selten'); J. Sahlberg cited it for N Karelia (1876: 'Nordligast har jag funnit den vid Soukelo i Ryska Lappmarken (6640')'), 'Peninsula Kola, Petschora' (1899); Jakobson (1909) summarized records for 'Arkhangelsk Government (Lapponia, Pechora), <...> Olonets Government, St.-Petersburg Government, Kiev Government'. Puthz (Pisanenko \& Puthz 1991) mentioned that 'Aus der europäischen Sowjetunion war diese Art Horion 1963 unbekannt', but, besides the records named above, Puthz (1971a) himself cited an additional find for the Leningrad Area ('Viipuri'=Vyborg); Tichomirova (1982) recorded the species for the Moscow Area. The sketchy figure of an aedeagus provided by Bordoni (2004) for S. nitens from Italy ('Veneto: Verona') seems to belong to a specimen of another species. Unfortunately the specimen which has been figured by Bordoni is not found in Museo Civico di Storia Naturale, Verona (A. Zanetti pers. comm.); thus, the Italian records of the species require a confirmation. The new material proves that the species is widely distributed in W \& C Siberia. The E Siberian records by Poppius (1909a: 'An der mittleren Lena weit verbreitet, aber einzeln. <...> Olekminsk, Ytyk-haja, Önkyr-yrjä, Ust-Aldan, zwischen Ust-Aldan und Batylym, Shigansk') have been confirmed by neither subsequent writers nor the new material. The aforecited material from W Evenkia seems to provide the easternmost verified records of the species until now. The shape of the aedeagus of the male from Evenkia corresponds well to that of the specimen from European Russia (see Fig. 1A-D).

Stenus (Nestus) raddei Ryvkin, 1987

(Fig. 2A, B)

Stenus (Nestus) raddei Ryvkin, 1987: 156.

Stenus raddei - Herman 2001: 2365.

Stenus (Nestus) raddei - Naomi \& Puthz 1994: 219.

Stenus (s. str.) raddei - Smetana 2004: 561.

\section{Material examined}

RUSSIA: 1 , Maritime Province, Lazovskiy Nature Reserve, $10 \mathrm{~km} \mathrm{~W}$ of Preobrazheniye, swampy meadow, $\mathrm{t}=20^{\circ}, \mathrm{pH}=5.4,17$ Aug. 1986, A. Shatrovskiy leg. (AR); 1 ô, Amur Province, Selemdzhinskiy District, near Selemdzhinsk, 270-280 m a.s.1., 12 Aug. 1976, E.M. Veselova \& A.B. Ryvkin leg. (AR); 1 ㅇ, Amur Area, Selemdzhinskiy District, Norskiy Reserve, near Fevral'sk, 268th km of BelogorskFevral'sk road, Tikhiy rill, $275 \mathrm{~m}$ a.s.1., mosses and plant debris between sedge \& gramineous tussocks among Alnus sp., Salix sp., Spiraea sp. with Sphagnum squarrosum, Sph. spp., etc., 8 Oct. 2008, A.B. Ryvkin leg. $<?>$ (AR); $3 \hat{\partial} \hat{\partial}, 4$ q 9 , Amur Area, Selemdzhinskiy District, Norskiy Nature Reserve, Selemdzha River basin near Dvadtsatikha cordon, open swamp: Carex sp., Spiraea spp., Geranium sp., Vicia sp., Poaceae gen. sp., Salix spp., Filipendula palmata, sparse Plagiomnium sp., Sphagnum sp., Polytrichum sp., etc., 4 Aug. 2004, A.B. Ryvkin leg. (AR); 1 \%, same locality, mosses and plant debris 
among tussocks at small open swamp with Carex spp. and Poaceae gen. sp., 10 Aug. 2004, A.B. Ryvkin leg. (AR); 3 $\widehat{\jmath}, 3$ 웅, Amur Area, Selemdzhinskiy District, Norskiy Nature Reserve, Selemdzha River basin, $1.5 \mathrm{~km}$ NE of Dvadtsatikha cordon, banks and burnt flood-plain of a rill inflowing to lake, $222 \mathrm{~m}$ a.s.1., mosses and leaf litter among Carex spp., Alnus sp., Salix spp., Padus sp., undergrowth of Betula spp. and Populus tremula, etc., 18 Aug. 2006, E.M. Veselova \& A.B. Ryvkin leg. (AR); 6 § $\partial, 4$ 우, Amur Area, Selemdzhinskiy District, Norskiy Nature Reserve, Selemdzha River basin, $2 \mathrm{~km} \mathrm{NE}$ of Dvadtsatikha cordon, open swamp near lakeside: plant debris and sparse mosses among tussocks with

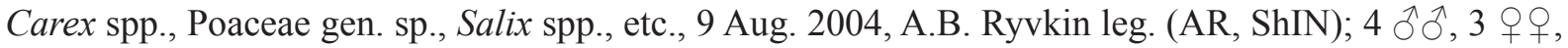
Amur Area, Selemdzhinskiy District, Selemdzha River basin, flood-plain of Aldikon River near Norsk, mosses and leaf litter under Salix sp. and along the edge of open sedge-gramineous tussock swamp,

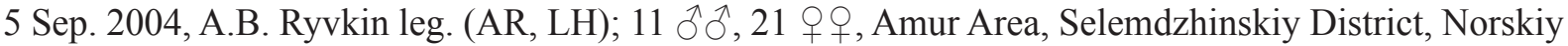
Nature Reserve, Nora River basin near Maltsevskiy cordon, mosses and plant debris on swampy floodplain lakeside: Carex spp., Calamagrostis sp., Filipendula palmata, Geranium sp., Comarum palustre, Spiraea sp., Salix sp., Sphagnum ? girgensohnii, Sph. squarrosum, Polytrichum commune, P. sp., Climacium sp., Iris sp., etc., 28 Aug. 2004, A.B. Ryvkin leg. (AR); $1 \hat{\jmath}$, Amur Area, Selemdzhinskiy District, Norskiy Nature Reserve, rill (left confluent of Nora River) up-stream of Gryashchinskaya Mt., true mosses and plant debris among Carex spp., Poaceae gen. sp., Alnus sp., Padus sp., Salix sp., etc.,

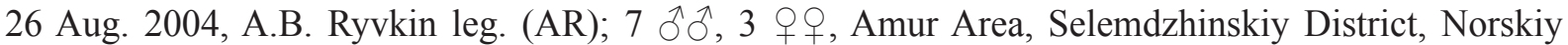
Nature Reserve, Nora River basin, $1.5 \mathrm{~km}$ up-stream of Gryashchinskaya Mt., mosses and plant debris on open swamp along lake side: tussocks of Carex spp. and Poaceae with Sphagnum ? girgensohnii, Sph. ? angustifolium, Sph. ? magellanicum, Sph. squarrosum, Sph. spp., Chamaedaphne calyculata, Vaccinium uliginosum, sparse Ledum palustre, Salix sp., Betula ? fruticosa, etc., 24 Aug. 2004, A.B. Ryvkin leg. (AR); 1 ऽ, 3 9 ㅇ, same locality, moss and plant debris on Carex-Eriophorum swamp with Chamaedaphne calyculata, gramineous admixture, Comarum palustre, Salix sp., Vaccinium

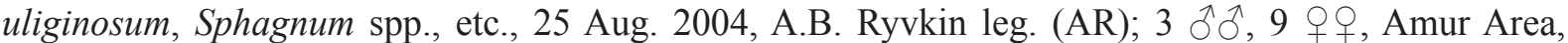
Selemdzhinskiy District, Norskiy Nature Reserve, Nora River basin, 2 km up-stream of Gryashchinskaya Mt., mosses and plant debris on small open swamp on high flood-plain: tussocks of Calamagrostis sp. and Carex spp. with Sphagnum ? girgensohnii, Sph. squarrosum, Sph. centrale, Sph. spp., Rubus arcticus,

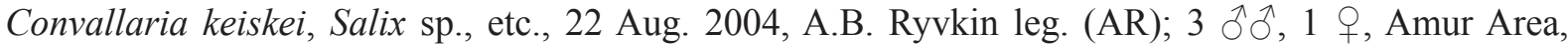
Selemdzhinskiy District, Norskiy Nature Reserve, Nora River basin near Meunskiy cordon, mosses and plant debris among Carex spp. \& Poaceae with Comarum palustre, Sphagnum squarrosum, Sph. spp., Aulacomnium sp. and other true mosses near side of flood-plain lake and swampy road, 13 Aug. 2004, A.B. Ryvkin leg. (AR); 1 ${ }^{\lambda}$, same locality, swamp with sedge tussocks, Poaceae gen. sp., Sphagnum squarrosum, Sph. spp., etc., 14 Jul. 2005, A.B. Ryvkin leg. (AR); 3 ồ, Amur Area, Selemdzhinskiy District, Norskiy Nature Reserve, Nora River basin near Meun River mouth, small swamp with Carex

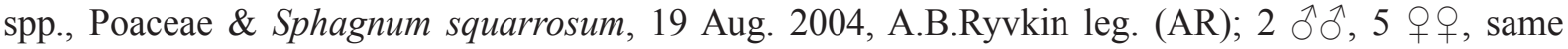
locality \& biotope, 20 Aug. 2004, A.B. Ryvkin leg. (AR); 1 \&, Amur Area, Selemdzhinskiy District, Norskiy Nature Reserve, Meun River mouth, plant debris among Carex spp. \& Equisetum sp. and leaf litter under Salix spp. \& Alnus sp. on low bank of creek, 21 Aug. 2004, A.B. Ryvkin leg. (AR); 1 万, Amur Area, Selemdzhinskiy District, Norskiy Nature Reserve, Burunda River bank $3 \mathrm{~km}$ up-stream of mouth, $215 \mathrm{~m}$ a.s.l., mosses and plant debris among sedges etc. and on shingle bed, 3 Aug. 2006, E.M.Veselova \& A.B. Ryvkin leg. (AR); 1 \&, Amur Area, Selemdzhinskiy District, Norskiy Nature Reserve, Burunda River near Ozyornyi Rill mouth, mosses and plant debris among sedge-gramineous tussocks on very gentle slope near intermittent channel: Carex spp., Calamagrostis sp., Polytrichum sp.

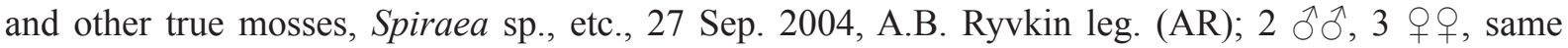
locality \& biotope. 28 Sep. 2004, A.B. Ryvkin leg. (AR); $5 \hat{\jmath}, 1$, same locality, plant debris and mosses among tussocks of Carex spp. and Poaceae with Spiraea sp., Vicia sp., small true mosses, etc. on

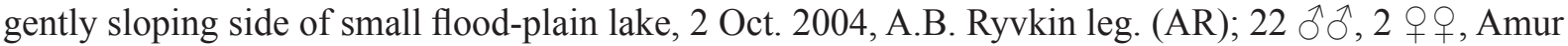
Area, Selemdzhinskiy District, Norskiy Nature Reserve (buffer zone), Burunda River basin, 1.5-2 km NW of Burunda cordon, mosses and plant debris on swamp and among sedge-gramineous tussocks in 

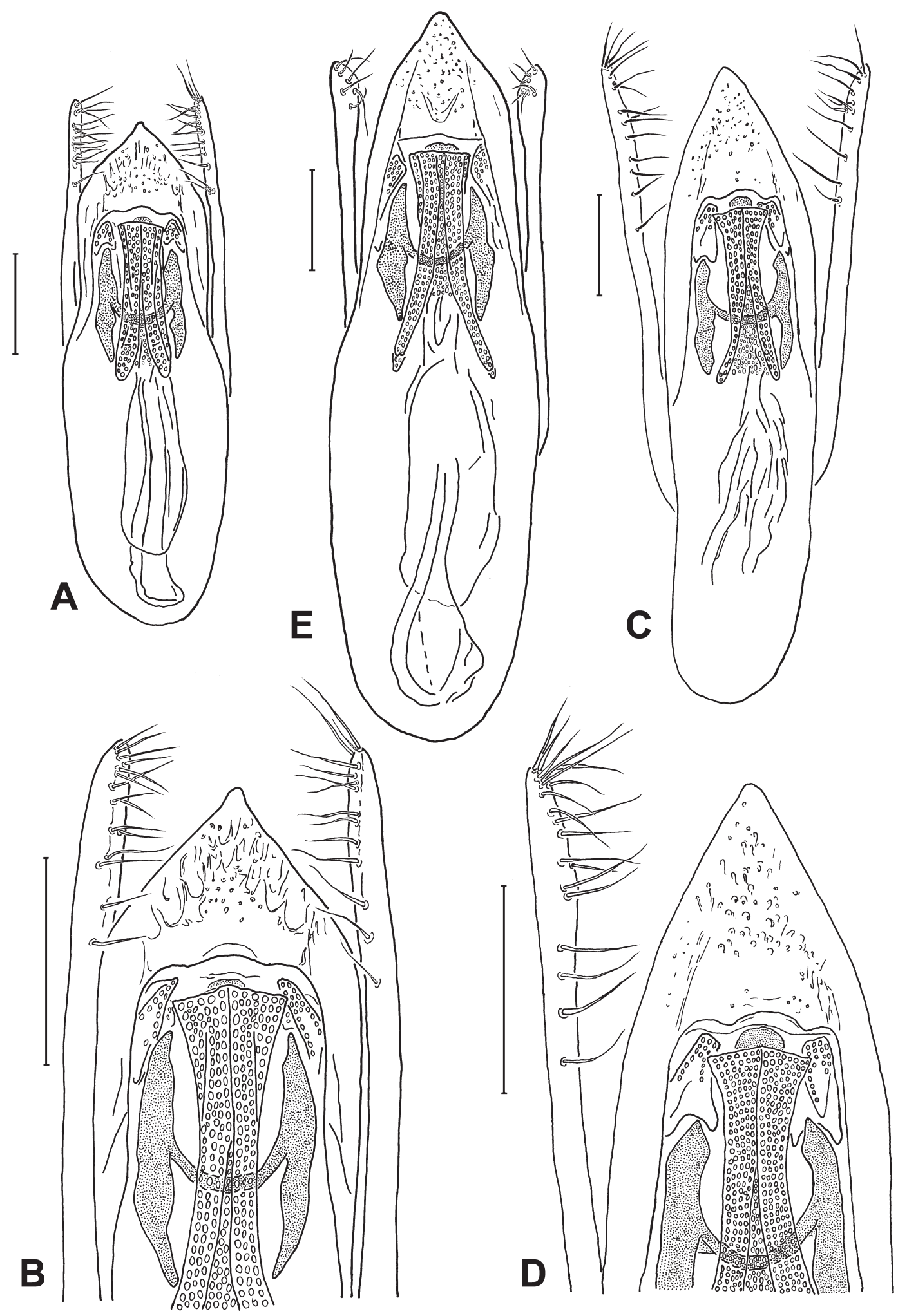

Fig. 2. - A-B: Stenus (Nestus) raddei Ryvkin, 1987: đ̊ (Russia: Magadan Area: Sibit-Tyhellakh). A. Aedeagus, ventral view. B. Apical part of aedeagus, ventral view. - C-D: S. (N.) illotulus Puthz, 1972: $\widehat{\delta}$ (Russia: Amur Area: Zeyskiy Nature Reserve). C. Aedeagus, ventral view. D. Apical part of aedeagus, ventral view. Right paramere removed. - E. S. (N.) confusus J. Sahlberg, 1876: đ̊ (Russia: Kalinin Area: Putilovo). Aedeagus, ventral view. - Scales $=0.1 \mathrm{~mm}$. 
sparse growth of burnt Betula platyphylla and Larix gmelinii near Kamyshovoye Lake with Chamaedaphne calyculata, Comarum palustre, Vaccinium uliginosum, Ledum palustre, Sphagnum spp., Polytrichum sp., Hypnum sp., etc., 19 Sep. 2004, A.B. Ryvkin leg. (AR); 1 + , Amur Area, Selemdzhinskiy District, Norskiy Nature Reserve (buffer zone), Burunda River basin, $1.5 \mathrm{~km}$ NW of Burunda cordon, sedge-gramineous swamp near rill, and also mosses and leaf litter among Carex spp. and under Alnus sp. on rill bank, 10 Sep. 2004, A.B. Ryvkin leg. (AR); 1 क, same locality, plant debris among Carex sp. under sparse Alnus sp. with young growth of Larix gmelinii along rill bank near road, 18 Sep. 2004, A.B. Ryvkin leg. (AR); 1 q, Amur Area, Selemdzhinskiy District, Norskiy Nature Reserve (buffer zone), Burunda River basin, 0.5-1 km NW of Burunda cordon, plant debris and small true mosses among Carex spp. \& Poaceae (+sweeping) along rill bank, 12 Sep. 2004, A.B. Ryvkin leg. (AR); 3 q $q$, Amur Area, Selemdzhinskiy District, Norskiy Nature Reserve (buffer zone), Burunda River basin near Burunda cordon, plant debris and mosses (+sweeping) on swampy sides of flood-plain lake: Carex spp., Poaceae gen. sp., Spiraea sp., sparse Climacium sp., Hypnum sp., Sphagnum sp., Polytrichum sp., etc., 9 Sep. 2004, A.B. Ryvkin leg. (AR); 1 O, 3 우, Amur Area, Selemdzhinskiy District, Norskiy Nature Reserve (buffer zone), Burunda River basin, $1 \mathrm{~km} \mathrm{SE}$ of Burunda cordon, mosses and plant debris among sedge tussocks on wide Carex-Eriophorum-gramineous swamp with small true mosses, Sphagnum squarrosum, Sph. sp., Ledum palustre, Vaccinium uliginosum, sparse Salix sp., young growth of Betula sp., etc., 21 Sep. 2004, A.B. Ryvkin leg. (AR); 1 , Amur Area, Selemdzhinskiy District, Norskiy Nature Reserve (buffer zone), Burunda River basin, $2.5 \mathrm{~km} \mathrm{SE}$ of Burunda cordon, small true mosses and plant debris among Carex spp. on sand, small shingles and clay along dried ephemeral stream near border of complex swamp, 21 Sep. 2004, A.B. Ryvkin leg. (AR); 1 \&, Khabarovsk Territory, Okhotsk, Airport, litter in larch forest, 15 Jul. 1987, V.V.Zherikhin \& I.D.Sukacheva leg. (AR); 1 q, Khabarovsk Territory, Okhotskiy District, Khetana River (confluent of Amka River, Ulya River basin), 7 km up-stream of river mouth, 31 Jul. 1985, V. Zherikhin, A. Rasnitsyn \& D. Shcherbakov leg. (AR); 1 , same locality \& collectors, 19 Aug. 1985 (AR); 1 ㅇ, Khabarovsk Territory, Verkhnebureinskiy District, 20th km of Chegdomyn-Urgal road, mosses and plant debris on swampy sides of wayside lake with Carex spp., Poaceae gen. sp., Ledum palustre, Chamaedaphne calyculata, Vaccinium uliginosum, Spiraea sp., Sphagnum spp., undergrowth of Betula platyphylla and Larix gmelinii, 6 Oct. 2006, A.B. Ryvkin leg. (AR); 1 O, Khabarovsk Territory, Verkhnebureinskiy District, Bureinskiy Nature Reserve (buffer zone) near 'Strelka' cordon, $550 \mathrm{~m}$ a.s.l., mosses, leaf litter and drift on banks of old channel of Umal'ta-Makit River: Sphagnum spp., Polytrichum sp., Carex spp., Poaceae gen. spp., etc., 30 Sep. 2006, A.B. Ryvkin leg. (AR); 1 \& , Khabarovsk Territory, Verkhnebureinskiy District, Bureinskiy Nature Reserve, 'Strelka' cordon, mari (bog), 28 Aug. 2006, A. Barševskis \& U. Valainis leg. (DUL); $3 \widehat{\jmath}$ ऽ, 4 우, Khabarovsk Territory, Verkhnebureinskiy District, Bureinskiy Nature Reserve, Pravaya Bureya River basin near Medvezhye winter hut, $1050 \mathrm{~m}$ a.s.l., mosses and litter in slope sparse larch forest with Sphagnum spp., Rubus chamaemorus, Ledum palustre, Ptilium crista-castrensis, Pleurozium schreberi, Polytrichum spp., Carex ? globularis, etc., 29 Jul. 2007, A.B. Ryvkin leg. (AR); 1 o, same locality, 1100-1240 m a.s.l., mosses, lichens and litter near rill in upper part of slope and on bald mountain crest: Picea ajanensis, Pinus pumila, Rhododendron aureum, Duschekia sp., Larix gmelinii, Sphagnum spp., Ptilium crista-castrensis, Pleurozium schreberi, Hylocomium splendens, Rubus chamaemorus, Carex ? globularis, etc., 29 Jul. 2007, A.B. Ryvkin leg. (AR); 1 + , Khabarovsk Territory, Verkhnebureinskiy District, left side of Olga River valley $3 \mathrm{~km}$ up-stream of Sofiysk, $880 \mathrm{~m}$, mosses, leaf litter \& plant debris on gentle slope near rill amid mari with hummocks of Sphagnum spp., Carex? globularis, C. spp., Poaceae gen. spp., Ledum sp., Vaccinium uliginosum, V. vitis-idaea, Polytrichum sp., Hypnum sp., Hylocomium splendens, Pleurozium schreberi, Ptilium crista-castrensis, Betula spp., etc., 19 Aug. 2008, A.B. Ryvkin leg. (AR); 1 đ̂, Khabarovsk Territory, Verkhnebureinskiy District, Ust'Urgal Nature Park (project), near Semicha River mouth, SE side of old channel, $280 \mathrm{~m}$ a.s.l., moss and plant debris in larch-Ledum sparse forest with Betula divaricata, Vaccinium uliginosum, Chamaedaphne calyculata, Sphagnum spp., Polytrichum spp., Dicranum spp., Cladonia spp., Eriophorum sp., Carex sp., etc., 29 Aug. 2009, A.B. Ryvkin leg. (AR); 2 우, Khabarovsk Territory, Verkhnebureinskiy District, 
Dublikanskiy Nature Refuge, right side of Dublikan River valley, $1 \mathrm{~km}$ up-stream of cordon, NNW slope of bald mountain, $400 \mathrm{~m}$ a.s.1., mosses and litter on stones with pika burrows (permafrost!): Pleurozium schreberi, Hylocomium splendens, Ptilium crista-castrensis, Sphagnum spp., Ledum sp., Vaccinium vitis-idaea, Carex sp., Poaceae gen. sp., etc. among Larix gmelinii, Betula platyphylla, Alnus sp., Duschekia sp., undergrowth of Abies nephrolepis and Picea ajanensis, 27 Aug. 2008, A.B. Ryvkin

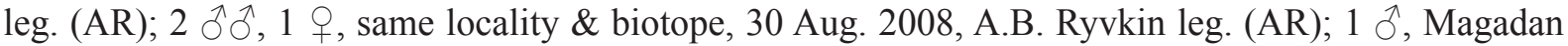
Area, Ten'kinskiy District, near Sibit-Tyhellakh, peat-moss stripe, 28 May-07 Jun. 1982, D.I. Berman leg. (AR); 2 §ิ, 1 ㅇ, Magadan Area, Ten'kinskiy District, near Sibit-Tyhellakh, Jack London Lake, Sphagnum swamp on alluvial cone, 18 Aug. 1984, K.Yu. Eskov leg. (AR); 1 +, Magadan Area, Ten'kinskiy District, near Sibit-Tyhellakh, 'Aborigen' field research station, alder shrubs near rill: in Sphagnum hillock, 24 Aug. 1984, K.Yu. Eskov leg. (AR); 1 +, Magadan Area, Ten'kinskiy District, Detrin River basin, Vakkhanka Rill, northern swamp, soil traps, 30 May-9 Jun. 1981, S.P. Bukhkalo leg. (AR); 1 ô, same locality, swamp near landing place, soil traps, $7-17$ Jun. 1982, S.P. Bukhkalo leg. (AR); 1 ${ }^{\lambda}$, same locality, swamp on glacial drift, 6-16 Jun. 1983, S.P. Bukhkalo leg. (AR); 1 q, same locality, peat-moss stripe, 7-17 Jun. 1983, S.P. Bukhkalo leg. (AR).

\section{Remarks}

This species was originally described based on two males (HT: [China:] Island in Sungari River; PT: [Russia: S Maritime Province:] Kedrovaya Pad' [Nature Reserve]); it was later cited for Hokkaido, Japan, and 'Amur region [without more precise locality]' (Naomi \& Puthz 1994). It should be noted that neither Herman (2001) nor Smetana (2004) mentioned the Chinese occurrence of this species in their respective catalogues. Both authors seem to have misinterpreted the terra typica as a Russian territory. Based on the new material above, $S$. raddei proves to be a very common species distributed widely in the Russian Far East.

Stenus (Nestus) illotulus Puthz, 1972

(Fig. 2C, D)

Stenus (Nestus) illotulus Puthz, 1972b: 169.

Stenus illotulus - Puthz 1972d: 107. — Shavrin \& Puthz 2007: 120.

Stenus (Nestus) illotulus - Ryvkin 1987: 159.

\section{Material examined}

RUSSIA: 1 , Tuva, Todjenskiy District, Azas Nature Reserve, near cordon at Azas Lake, $940 \mathrm{~m}$ a.s.l., left bank of channel: sedge-gramineous bog (+ Trollius sp. etc.) with tussocks, 15 Jun. 1991,

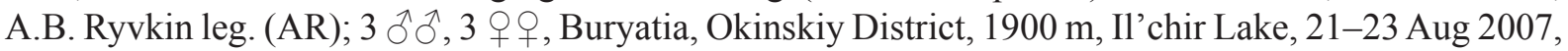

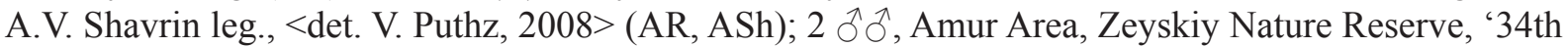
km' cordon, Gulik River valley, in litter, 23 Jun. 1978, V.V. Belov \& S.A. Kurbatov leg. (AR); 1 ô, Amur Area, Selemdzhinskiy District, Byssa River 10 km below 'Tyoplyi Klyuch' spa, 290 m a.s.l., leaf litter among tussocks with Spiraea sp., Calamagrostis sp., Carex spp., etc. in high flood-plain, 3 Jun. 2007, E.M. Veselova \& A.B. Ryvkin leg. (AR); 1 ô, 1 q, Amur Area, Selemdzhinskiy District, Byssa River basin, bottom part of mountainside NW of 'Tyoplyi Klyuch' spa, 370 m a.s.l., litter under Alnus sp. with Spiraea sp., undergrowth of Betula platyphylla, Trientalis europaea, Poaceae gen. spp., Rubus arcticus, etc., 22 Jun. 2007, E.M. Veselova \& A.B. Ryvkin leg. (AR); 1 §, 1 q, Amur Area, Selemdzhinskiy District, Norskiy Nature Reserve, Selemdzha River basin, $2 \mathrm{~km}$ NE of Dvadtsatikha cordon, open swamp near lakeside: plant debris and sparse mosses among tussocks with Carex spp., Poaceae gen. sp., Salix spp., etc., 9 Aug. 2004, A.B. Ryvkin leg. (AR); 1 Oे, Amur Area, Mazanovskiy District, Nora River mouth, $210 \mathrm{~m}$ a.s.l., mosses and leaf litter on natural levee and in flood-plain forest with Alnus 
sp., Salix spp., Padus sp., ferns, Poaceae gen. spp., Carex spp., Smilacina davurica, etc., 6 Aug. 2006, E.M. Veselova \& A.B. Ryvkin leg. (AR); 2 우, Amur Area, Selemdzhinskiy District, Norskiy Nature Reserve, Nora River basin near Maltsevskiy cordon, mosses and plant debris on swampy flood-plain lakeside: Carex spp., Calamagrostis sp., Filipendula palmata, Geranium sp., Comarum palustre, Spiraea sp., Salix sp., Sphagnum ? girgensohnii, Sph. squarrosum, Polytrichum commune, P. sp., Climacium sp., Iris sp., etc., 28 Aug. 2004, A.B. Ryvkin leg. (AR); 5 우, Amur Area, Mazanovskiy District, Nora River basin, Sorokavyorstnaya channel, upper reaches of Zolotoy Rill, $270 \mathrm{~m}$ a.s.1., mosses and plant debris along edge of slope swamp near mountain crest: Eriophorum sp., Chamaedaphne calyculata, Vaccinium uliginosum, Ledum palustre, Rhododendron sp., Betula? fruticosa, B. platyphylla, Alnus sp., Oxycoccus sp., etc., 28 Jul. 2006, E.M. Veselova \& A.B. Ryvkin leg. (AR); 1 ô, Amur Area, Mazanovskiy District, Nora River basin, Sorokavyorstnaya channel, foot of S slope of Sosnovaya Mountain, $215 \mathrm{~m}$ a.s.l., flood-plain birch forest with Betula platyphylla, Alnus sp., Padus sp., tussocks of Carex spp. and Poaceae gen. spp., Pteridium aquilinum, Convallaria keiskei, Thalictrum sp., Equisetum sylvaticum, etc., 29 Jul. 2006, E.M. Veselova \& A.B. Ryvkin leg. (LH); 3 $\widehat{\jmath}$, Amur Area, Selemdzhinskiy District, Norskiy Nature Reserve, Nora River, $0.5 \mathrm{~km}$ up-stream of Gryashchinskaya Mt., mosses and leaf litter under Betula platyphylla and Larix gmelinii with Calamagrostis sp., Equisetum sylvaticum, E. pratense, Maianthemum bifolium, Polygonatum sp., etc. in burnt forest along river bank, 27 Aug. 2004, A.B. Ryvkin leg. (ShIN); 2 $\widehat{\jmath}, 1$ ๆ, Amur Area, Selemdzhinskiy District, Norskiy Nature Reserve, Nora River basin, $1.5 \mathrm{~km}$ up-stream of Gryashchinskaya Mt., mosses and plant debris on open swamp along lake side: tussocks of Carex spp. and Poaceae with Sphagnum ? girgensohnii, Sph. ? angustifolium, Sph. ? magellanicum, Sph. squarrosum, Sph. spp., Chamaedaphne calyculata, Vaccinium uliginosum, sparse Ledum palustre, Salix sp., Betula ? fruticosa, etc., 24 Aug. 2004, A.B. Ryvkin leg. (AR); 1 ð, Amur Area, Selemdzhinskiy District, Norskiy Nature Reserve, Nora River basin near Meunskiy cordon, mosses and plant debris among Carex spp. \& Poaceae with Comarum palustre, Sphagnum squarrosum, Sph. spp., Aulacomnium sp. and other true mosses near side of flood-plain lake and swampy road, 13 Aug. 2004, A.B. Ryvkin leg. (AR); 1 Oे, Amur Area, Selemdzhinskiy District, Norskiy Nature Reserve, Nora River basin near Meunskiy cordon, mosses and plant debris at flood-plain of a rill: Alnus sp., Carex spp., Poaceae gen. sp., Trientalis europaea, Convallaria keiskei, Sphagnum squarrosum, Sph. spp., Hypnum sp., etc., 15 Aug. 2004, A.B. Ryvkin leg. (AR); 1 +, Amur Area, Selemdzhinskiy District, Norskiy Nature Reserve (buffer zone), Burunda River basin, $1.5 \mathrm{~km}$ NW of Burunda cordon, plant debris among tussocks of Carex spp. under sparse Alnus sp. with young growth of Larix gmelinii along rill bank near road, 17 Sep. 2004, A.B. Ryvkin leg. (AR); 2 우, same locality and biotope, 18 Sep. 2004, A.B.Ryvkin leg. (AR); 1 , Amur Area, Selemdzhinskiy District, Norskiy Nature Reserve (buffer zone), Burunda River basin, $0.5-1 \mathrm{~km} \mathrm{NW}$ of Burunda cordon, plant debris and small true mosses among Carex spp. \& Poaceae (+sweeping) along rill bank, 12 Sep. 2004, A.B. Ryvkin leg. (AR); 1 ग, same locality, mosses and plant debris among sedge tussocks with Spiraea sp., ? Aulacomnium sp., Sphagnum squarrosum, Sph. ? girgensohnii, small true mosses, etc., 5 Oct. 2004, A.B. Ryvkin leg. (AR); 1 ग, Amur Area, Selemdzhinskiy District, Norskiy Nature Reserve (buffer zone), Burunda River basin, 1.5-2 km SE of Burunda cordon, mosses and leaf litter under Betula platyphylla with Ledum palustre, Chamaedaphne calyculata, Salix sp., Sphagnum spp., Hypnum sp., Poaceae gen. sp., etc., 6 Oct. 2004, A.B. Ryvkin leg. (AR); 1 ㅇ, Amur Area, Selemdzhinskiy District, Norskiy Reserve, near Fevral'sk, 268th km of Belogorsk-Fevral'sk road, Tikhiy rill, $275 \mathrm{~m}$ a.s.l., mosses and plant debris between sedge \& gramineous tussocks among Alnus sp., Salix sp., Spiraea sp. with Sphagnum squarrosum, Sph. spp., etc., 8 Oct. 2008, A.B. Ryvkin leg. (AR); 1 +, Khabarovsk Territory, Verkhnebureinskiy District, lower reaches of Verkhniy Mel'gin River near 1st rapid, 300-350 m a.s.l., moss and litter on steep rocky SE slope with Betula platyphylla, Abies nephrolepis, Larix gmelinii, Acer ukurunduense, Rhododendron dauricum, Poaceae gen. spp., Carex sp., Vaccinium vitis-idaea, Linnaea borealis, Maianthemum bifolium, Trientalis europaea, Ledum sp., Alnus sp., ferns, Equisetum pratense, Hylocomium splendens, Sphagnum girgensohnii, Dicranum sp., Ptilium crista-castrensis, Polytrichum commune, etc., 20 Aug. 2009, A.B. Ryvkin leg. (AR); 1 ○̂, 1 क , Khabarovsk Territory, Verkhnebureinskiy District, Ust'-Urgal 
Nature Park (project), near Semicha River mouth, SE side of old channel, $280 \mathrm{~m}$ a.s.l., leaf litter and moss in birch forest with Larix gmelinii, Ledum sp., Vaccinium vitis-idaea, Poaceae gen. spp., Carex sp. by edge of larch-Ledum sparse forest, 29 Aug. 2009, A.B. Ryvkin leg. (AR).

\section{Remarks}

This very nice and distinctive species was described based only on the male holotype, from Southern Cis-Baikalia ('Südsibirien: Tunkun-Sajan'). In the same year, Puthz (1972c), based on two specimens deposited in the Helsinki Museum, reported S. illotulus for the N Lena basin: 'Shigansk [=Zhigansk] und $\langle\ldots\rangle$ Ust-Aldan'. The latter specimen is a male, and the sketch of the aedeagus that I recently received from Dr. Puthz proves that it is unlikely that it belongs to S. illotulus. I think it necessary to accumulate additional material from the $\mathrm{N}$ Lena basin to give an adequate interpretation of these specimens. Shavrin \& Puthz (2007) provided records for the Irkutsk Area (I have seen the specimen) and SE Buryatia; the mention of the Krasnoyarsk Territory and N Mongolia in distributional remarks by the named authors was not accompanied by a reference to any material. The remark concerning the Krasnoyarsk Territory was a misprint (A. Shavrin pers. comm.). The remark for N Mongolia is based on

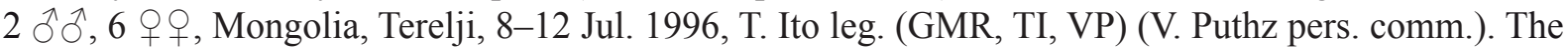
new data cited above from the Khabarovsk Territory, Amur Area, and Tuva Republic widen the known range of S. illotulus as regards both its SW and its E limits. Stenus illotulus, as well as S. raddei, inhabits moss and leaf litter in habitats moist to moderately wet, but is as a rule less abundant than the latter in those communities.

\section{Stenus (Nestus) caseyi Puthz, 1972}

(Fig. 5F)

Stenus (Nestus) caseyi Puthz, 1972b: 172.

Stenus caseyi - Puthz 1975b: 125.

Stenus (s. str.) caseyi - Campbell \& Davies 1991: 110.

\section{Material examined}

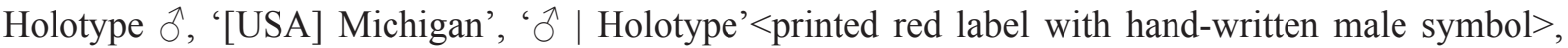
'Stenus $\mid$ caseyi $\mid$ n. sp. $\mid$ det. V. Puthz 1971 '<standard Puthz's determinative label $>$ (MNHG).

USA: 1 o, 4 우, Mich. Berrien Co., 2 mi. N. New Buffalo, Gallien R. W bank, 2 Nov. 1980, litter in marsh, L.E. Watrous leg. (FMNH); $4 \hat{\jmath}, 1$ q, Wisconsin, Kenosha Co., Carol Beach, Chiwaukee Prairie, 24 Oct. 1980, FMHD\#80-35 litter in wet grassy pockets, L. Watrous \& W. Suter leg. (FMNH);

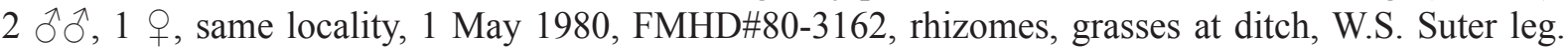
(FMNH); 2 우, same locality, 19 Mar. 1974, W. Suter leg., FM(HD)\#74-49, Ber.: litter in semi-dry ditch, WS\#74-22b (FMNH); 1 o, same locality, 4 Jun. 1974, W. Suter leg., FM(HD)\#74-163, Ber.: Marsh floor at grass hummocks, WS\#74-44a (FMNH); 2 స̂, 1 ㅇ, same locality, 10 May 1975, W. Suter leg., FM(HD)\#75-189, Ber.: grassy moss clumps marsh area (FMNH); $1 \hat{\jmath}$, same locality, 26 Apr. 1974, W. Suter leg., FM(HD)\#74-66, Ber.: floor at cattails, WS\#74-30D (FMNH); 1 o, same locality, 26 Apr. 1974, W. Suter leg., FM(HD)\#74-65, Ber.: sandy soil at water table, WS\#74-30C (FMNH); 1 q, same locality, 11 Sep. 1976, W. Suter leg., FM(HD)\#76-160, WS\#76-65b, temporary pond, floor peat (FMNH).

\section{Remarks}

The species was originally described from Michigan, USA, has subsequently been reported from Quebec, Canada (Puthz 1975b) and also cited for the Canadian provinces Ontario, Quebec, New Brunswick and Nova Scotia (Campbell \& Davies 1991) without citing material. The new data above widen the range to 
include Wisconsin. Since the genital preparation of the holotype had been somewhat deformed owing to excessively quick dehydration, the figure of the aedeagus in the original description was not quite adequate. Based on the available material, I herein provide a new figure for this structure (see Fig. 5F).

\section{Stenus (Nestus) brivioi Puthz, 1972}

Stenus (Nestus) brivioi Puthz, 1972c: 12.

Stenus brivioi - Puthz 1972b: 171 (note). — Puthz 1975a: 124.

Stenus (s. str.) brivioi - Campbell \& Davies: 110.

Stenus vinnulus - Casey 1884: 112 (pars).

\section{Material examined}

CANADA: $1 \hat{\jmath}$, Alberta, $5 \mathrm{~km}$ W. Ashmont Rte. 28A, Camas-lily marsh, 12 Jun. 1977, G.E. Ball

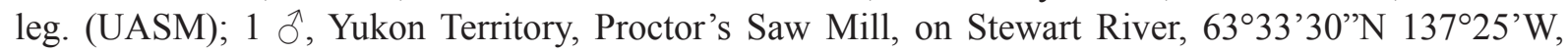
collected by treading grass tussocks 2'-8' from shore of pond, 3 Jul. 1977, R.E. Morlan, J.V. Matthews, R.E. Roughley leg. (Yukon Refugium Project) (UASM).

\section{Remarks}

Terra typica: USA: Michigan, Maryglade College, E. of Memphis, Macomb Co; Paratype: Eagle Har., L.[ake] Sup.[erior]. Puthz (1972a) attributed paralectotypes of S. vinnulus Casey, 1884 (see below) from White Fish Point to S. brivioi as well, and also reported the latter for Quebec, Canada (1975). Campbell \& Davies (1991), without citing material, reported S. brivioi for Alaska and most provinces of Canada (excluding British Columbia and Prince Edward Island).

\section{Stenus (Nestus) dolosus Casey, 1884}

Stenus dolosus Casey, 1884: 108.

Stenus dolosus - Puthz 1975a: 124.

Stenus (Nestus) dolosus - Puthz 1972b: 171.

Stenus (s. str.) dolosus - Bernhauer \& Schubert 1911: 157. — Campbell \& Davies 1991: 111.

Stenus jejunus Casey, 1884: 112.

Stenus villosus Casey, 1884: 109.

Stenus villosus - Casey 1892: 711.

Stenus (Nestus) villosus - L. Benick 1938: 266.

Stenus (s. str.) villosus - Bernhauer \& Schubert 1911: 168.

\section{Material examined}

CANADA: 1 q, Yukon Territory, 24 mi w Whitehorse, Alaska Hwy., 2000', 6051'N 136³6’W, 21 Jun. 1977, R.E. Morlan, J.V. Matthews, R.E. Roughley leg. (Yukon Refugium Project) (UASM).

USA: 1 + , 'Wisconsin. Kenosha Co.; Chiwaukee Prairie, Carol Beach. 26:IV:1974. Leg: W. Suter.', 'FM(HD)\#74-65. Ber.: sandy soil at water table. WS\#74-30C' <without top abdominal segments> (FMNH); 1 9, Ithaca, Cayuga Lake, 17 Aug. 1928, Dr. Rambousek leg., L. Hromadka det., 1979 (AR); 1 ô, Ottawa Co., Ohio, South Bath Is., Squaw Harbor, Put-in-Bay, washed-up eel-grass on sand beach, 2 Aug. 1979, S.W. Nichols leg. (FMNH); 1 ô, Michigan, Clinton Co., Rose L. Exp. Sta. ex mud edge, 
Typha marsh, 3 Sep. 1965, Thomas Hlavac leg. (FMNH); 1 ð’, 'Volga S.D. Edge of Pond. 5 May 1966. V.M. Kirk', 'N.M. Downie Colln. 1992 Acc. Z-18,343 FIELD MUSEUM' (FMNH); 1 §’, 'Rossie, N.Y. C-15 1965. N.M. Downie leg.', 'N.M. Downie Colln. 1992 Acc. Z-18,343 FIELD MUSEUM' (FMNH); 1 ㅇ, 'Tompkins Co, N.Y. F-15 1967. N.M. Downie leg.', 'N.M. Downie Colln. 1992 Acc. Z-18,343 FIELD MUSEUM' (FMNH).

\section{Remarks}

Terra typica: USA: 'Fort Garland, Colorado'; for S. villosus: 'California'; for S. jejunus: 'British Columbia'. Casey (1892) himself synonymized $S$. jejunus with $S$. villosus. Revising all three holotypes, Puthz (1972a) found them to be conspecific and placed S. villosus in synonymy with S. dolosus. Campbell \& Davies (1991) reported this species for Alaska and most provinces of Canada: Yukon Territory, Northwest Territories, Alberta, Saskatchewan, Manitoba, Ontario, Quebec and New Brunswick, without citing material.

\section{Stenus (Nestus) milleporus Casey, 1884 sp. propria}

Stenus milleporus Casey, 1884: 111.

Stenus milleporus - Casey 1892: 711.

Stenus illustris L. Benick, 1926: 263.

Stenus (Nestus) illustris - L. Benick 1938: 266.

Stenus odius Blackwelder, 1943: 213.

Stenus sectilifer Casey, 1884: 110.

Stenus sectilifer - Herman 2001: 2384.

Stenus (Nestus) sectilifer - Puthz 1972b: 171.

Stenus (s. str.) sectilifer - Bernhauer \& Schubert 1911: 167.

Stenus teter Notman, 1920: 699.

\section{Material examined}

USA: 2 qo+, Florida, Marion Co., 3 mi N Blichton, 26 Mar. 1976, C.W.O'Brien \& G.B.Marshall (FMNH).

\section{Remarks}

Originally described from the USA: 'New York, 3; St. Catharine Island, Georgia, 3; Florida, 7' <the digits refer to the number of specimens in the original type series>; the holotype of S. sectilifer: 'District of Columbia'. Stenus teter was described from Florida. Terra typica of S. illustris: Mexico; of S. odius: Cuba, Soledad near Cienfuegos. Acting as first reviser, Casey (1892) himself synonymized S. sectilifer with S. milleporus. However, Bernhauer \& Schubert (1911) mistakenly regarded S. milleporus as a junior synonym of S. sectilifer, and this treatment was repeated by Puthz (1972a) when placing S. teter, S. illustris, and S. odius in the synonymy of S. sectilifer. Under Articles 24.2.1 and 24.2.2 of ICZN, the valid name for the species under consideration is Stenus milleporus Casey, 1884. 


\section{Stenus (Nestus) canaliculatus Gyllenhal, 1827}

(Fig. 3D, E)

Stenus canaliculatus Gyllenhal, 1827: 501.

Stenus canaliculatus - Fauvel 1869: 492. — J. Sahlberg 1871: 415. — Hochhuth 1872: 154. — Fauvel 1873: 255. — J. Sahlberg 1876: 55, 323. — Eppelsheim 1880: 94. — J. Sahlberg 1880: 77. — Heyden 1881: 78. - Casey 1884: 115. - Fauvel 1889: 119. — Hamilton 1889: 114. — Eppelsheim 1893: 56. — Hamilton 1894a: 20. — Hamilton 1894b: 371. — Poppius 1899: 38. — J. Sahlberg 1900: 28. — Fauvel 1902: 75. — Jakovlev 1902: 112. — L. Benick 1921a: 148. — L. Benick 1924: 254. — Lebedev 1925: 134. - Iljin 1926: 225. — Szujecki 1958: 118. — Puthz 1970b: 174, 178. — Korge 1971: 6. — Puthz 1971c: 139. — Puthz 1973a: 27. — Uhlig \& Vogel 1981: 98. — Tichomirova 1982: 213. — Dubeshko 1984: 52. - Silfverberg 1988: $20<$ 'holotype' of congener Maeklin, 1853>. — Pisanenko \& Puthz 1991: 170, 174. — Puthz \& Zanetti 1995: 17. — Shavrin 2007: 141. — Shavrin \& Puthz 2007: 114 (pars).

Stenus (Nestus) canaliculatus - Rey 1884: 273. — Jakobson 1909: 481. — Poppius 1909a: 15. — Reitter 1909: 158. — Renkonen 1935: 29. — Palm 1961: 88. — Szujecki 1961: 34. — Puthz 1967b: 78. — Puthz 1971a: 82. — Puthz 1972a: 259. — Puthz 1972c: 12.— Tichomirova 1973: 173. — Shilov 1975: 58. — Ryvkin 1987: 159. — Ryvkin 1990: 168. — Kuznetsova 1991: 171. — Dauphin 1993: 188. — Ryabukhin 1999: 46. — Bordoni 2004: 120. — Semenov 2004: 11.

Stenus (s. str.) canaliculatus - Heyden 1898: 34. — Bernhauer \& Schubert 1911: 156. — Scheerpeltz 1933: 1150. — Campbell \& Davies 1991: 110. — Puthz 2001: 36.

Stenus affinis Stephens, 1833: 298.

$<$ ? $>$ Stenus congener Maeklin, 1853: 192.

$<$ ? >Stenus congener - Casey 1884: 114. — Hamilton 1889: 114. — L. Benick 1921b: 114.

$<$ ?>Stenus (s. str.) congener - Scheerpeltz 1933: 1152.

Stenus cordaticollis Leinberg, 1900: 187.

Stenus cordaticollis - J. Sahlberg 1900: 28.

Stenus (Nestus) cordaticollis - Jakobson 1909: 481.

Stenus (Nestus) foveifrons Rey, 1884: 272.

\section{Material examined}

FRANCE: 1 +, 'Caen (Ga) Raffray' <white rectangle>, 'Fvl[?Fauvel] Caen 67.' < green circle>, 'Fvl[?Fauvel] Stenus canaliculatus Gyll.' <white circle>, 'c. [ollection] of A. Jakovlev' <printed label> (ZIN).

POLAND: 1 ô, 'Silesia. 4106[=Stenus canaliculatus Gyll.] - 3' (ZIN); 1 †, 'Danzig', <underside> 'Kraatz.', 'St. canaliculatus Gyll.', 'c.[ollection] of A. Jakovlev' < printed label> (ZIN).

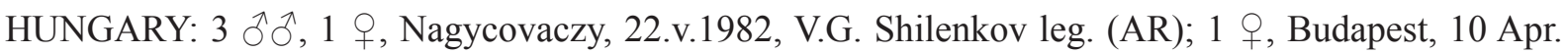
1982, V.G. Shilenkov leg. (ASh).

UKRAINE: 1 q, Podol'sk Government, Kamenets-Podol'sk, 12 Apr. 1911, V. \& I. Yakubovskiy leg. (ZIN); 1 ○े, ‘Odessa, Krivaya Balka. 4.iii.1920. D. Znojko leg.', 'Stenus (Nestus) morio Grav.?' (ZIN); 1 \&, Odessa, Fontanka, slope, lakeside, 4 Jul. 1970, S.Ya. Blinshtein leg. (AR); 1 , Nikolayev Area, Rybakovka, sandy seashore, 28 Jul. 1970, S.Ya. Blinshtein leg. (AR). 
RUSSIA: 1 đ̆, Kaliningrad Area, Rybachiy, 6 Jun. 1959, O.L. Kryzhanovskiy leg. (ZIN); 1 q, same locality and collector, 28 Jun. 1959 (ZIN); 1 ô, Petropol.[is]. (ZIN); 1 ㅇ, same locality, A. Moravitz

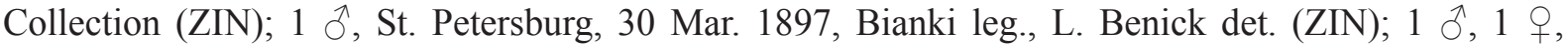
[St. Petersburg] Smolenskoye cemetery, 18 Aug. 1890 (ZIN); 1 o, same locality, 5 Apr. 1891 (ZIN);

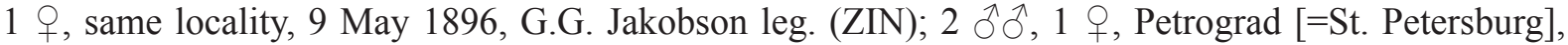
Smolenskoye cemetery, 11 Apr. 1920, A.A. Stackelberg leg., L. Benick det. (ZIN); 2 q, [St. Petersburg] Ekaterinhof, sifting, leaves, 2 Apr. 1921 (ZIN); 1 q, 'Small Islands on Neva River, Shlisselburg Uyezd [=District], 1 Jun. 1906. G.G. Jakobson leg.', 'canaliculatus' (ZIN); 1 q, Pargolovo, Finlandskaya Railway, 8 Aug. 1905, Somina leg., L. Benick det. (ZIN); 1 §̊, St. Petersburg Government, Tsarskosel'skiy Uyezd [=District], Orlinskoye Lake, 26 Apr. 1905, D. Glazunov leg. (ZIN); 1 q, [St. Petersburg Government], Luzhskiy District, Ploskoye, 7 Apr. 1906, Yu.I. Bekman leg. (ZIN); 1 Oे, [? St. Petersburg Government], Lesnoy, 12 Oct. 1897 (ZIN); 1 Oૈ, St. Petersburg Government, Luzhskiy Uyezd [=District], Preobrazhenskaya, 2 May 1905, D. Glazunov leg. (ZIN); 1 क, '[St.-Petersburg Government,] Yamburg. [skiy] u.[yezd] [= Yamburgskiy District]. 12.1912.-01.1913. A.P. Rimskiy-Korsakov leg.' (ZIN); 1 , Smolensk, bank of Dnepr River, on moist sand, 10 May 1993, M.Yu. Gildenkov leg. (SSTC); 1 q, Moscow Government, Klinskiy Uyezd [=District], Boblovo, 16 Apr. 1906, D. Smirnov leg. (ZIN); 1 q, Vladimir Area, Petushki, 1 May 1983, V.B. Semenov leg. (AR); 1 , same locality and collector, in litter, 21 Apr. 1984 (AR); 1 \%, Kostroma Area, Manturovskiy District, near Shilovo, Andromeda polifolia - Sphagnum magellanicum, Jun.1981, A.L. Tichomirova leg. (AR); 1 q, 'Urzhum, drift of Urzhumka River, 21.iii.1901, [L. Krulikovskiy leg.]', 'canaliculatus d. Bernh.[auer]', 'c.[ollection] of A. Jakovlev'<printed label $>$ (ZIN); 1 + , 'Urzhum, drift of Urzhumka River. 23.iii.1901. [L. Krulikovskiy leg.]', 'canaliculatus [?Bernhauer det.]', 'c.[ollection] of A. Jakovlev' <printed label> (ZIN); 1 ㅇ, 'Urzhum, drift of Urzhumka River. 24-25.iii.1901. L. Krulikovskiy leg.', 'c.[ollection] of A. Jakovlev' <printed label> (ZIN); 1 ก, 2 우, '[Urzhum,] drift of Urzhumka River. 09.iv.1906. L. Krulikovskiy leg.', 'canaliculatus det. Shakhonin', 'c.[ollection] of A. Jakovlev' <printed label> (ZIN); 2 우, 'Malmyzh, drift of Shoshma River. 03-04.iv.1899. L. Krulikovskiy leg.', 'canaliculatus',

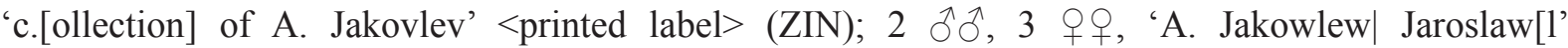
Government]', 'C.[ollection] of A. Jakovlev' , 'Stenus canaliculatus Gyll. d. Fauvel', 'c.[ollection] of A. Jakovlev' <printed label> (ZIN); 1 o, 1 o, 'Yaroslav[1']', <underside> 'Eppelsh[eim]', 'c.[ollection] of A. Jakovlev' <printed label> (ZIN); 1 o, '[Yaroslavl' Government,] Manikha, sifting from floating litter. 15.iv.1898.', 'Yaroslavskiy U.[yezd] [=District]. C.[ollection] of A. Jakowlew', 'Stenus carbonarius Gyll. [det. ?A.Jakowlew]' (ZIN); 1 + , Yaroslavl' Area, Nekouzskiy District, Borok, shore of reservoir, 6 Jun. 1979, E.M. Veselova leg. (AR); 1 q, 'Ryaz[an]'<white rectangle with thin black frame and species name> 'Stenus aterrimus Er.', 'C. [ollection] of A. Jakowlew' (ZIN); 1 § , 1 ㅇ, 'Riasan.' < printed label on red paper>, 'Stenus canaliculatus Gyll.' <black Indian ink in dubble box> (ZIN: ex coll. Semenov-Tian-Shanskiy); 1 + , Gremyachka, Dankovskiy Uyezd [=District], Ryazan' Government, 13 Aug. 1899, A. Semenov leg. (ZIN: ex coll. Semenov-Tian-Shanskiy); 1 q, same locality and collector, 19 Sep. 1901 (ZIN); 1 ㅇ, Tataria, Stolbishchevskiy District, collective farm '13 Let Oktyabrya', perennial grass, clover: in trap buckets, 1 Jun. 1956, Utrobina leg. (AR); 1 \%, Voronezh Area, Khopyorskiy Nature Reserve, near water, 5 Jun.? 1976. (AR); 1 Oे, Samara Area, Kinel'skiy District, Krasnosamarskoye Forestry, flood-plain of Samara River, 2 Jul. 1995, I.N.Goreslavets leg., 'Stenus sp-6 det. Goreslavetz I.N.' (IG); 1 + , Saratov Area, Khvalynsk, Sterkh River, 30 Jul. 1974, V.I. Lomakin leg. (AR); 1 q, [Rostov Area] Env. Novocherkassk, Razliv, 17 Apr. 1913, V. Kiseritzky leg. (ZIN); 1 + , Rostov Area, Nizshne-Kundryuchenskoye hunting farm, 2 Jun. 1990, V. Grebennikov leg. (ZMRU); 1 q, 'Sarepta. [18]95. Becker.', 'canaliculatus d. Bernh.[auer]', 'c.[ollection] of A. Jakovlev' <printed

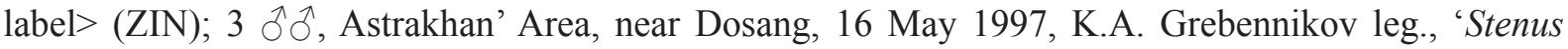
(Nestus) ? canaliculatus Gyll. K.A. Grebennikov det.1997' (KG); 1 q, Kalmyk ASSR, Lysyi Liman, 26 Jun. 1977, Arzanov leg. (ZMRU); 1 đ, Kalmykiya, Arshanya Zelmen', 3 Jun. 1980, N.S. Kalyuzhnaya leg. (KG); 1 , NW Caucasus [Krasnodar Territory], Malyi Tkhach Mt., 2000 m a.s.l., subalpine belt, clayey bank of puddle, 4 Jun. 1994, A.Yu. Solodovnikov leg. (AYuS); 1 q, Murmansk Area, Poyakonda, 
Biological Field Research Station of Moscow University, ejected Fucus, 14 Jun. 1979, E.M. Veselova leg. (AR); 1 q, same locality and collector, Alopecurus ventricosus, 13-17 Jul. 1979 (AR); 1 , near Solovetskiy Monastery, Bol'shoy Solovetskiy Island, 1893, G.G. Jakobson leg. (ZIN); 1 ô, Komi ASSR, Gibyu River, near Ukhta, fluvial terrace above flood-plain, in sod, 26 Jun. 1974, V.F. Shilov leg. (ZIN);

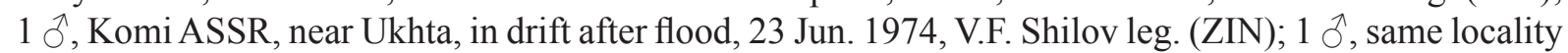
and collector, 5 May 1974 (ZIN); 1 ô, Sob' River basin, Bol'shoy Ural, Obdorsk [=Salekhard], 8 Jul. 1925, Fridolin leg. (ZIN); 1 ㅇ, Tyumen Area, Uvatskiy District, 10-11 km S of Gornoslinkino, steep right bank of Irtysh River up-stream of Tobolsk Field Research Station of Severtsov Institute, small mosses, plant debris, denuded loam among Tussilago farfara, Carex sp., Poaceae gen. sp., Fabaceae etc. near small rill draining by slope, 11 Jun. 2004, A.B. Ryvkin leg. (AR); 1 \&, Tyumen Area, Uvatskiy District, $11 \mathrm{~km} \mathrm{~S}$ of Gornoslinkino, clayish right bank of Irtysh River (narrow fold at rill mouth) upstream of Tobolsk Field Research Station of Severtsov Institute, in leaf litter and sweeping under Salix sp. with small true mosses, Marchantia sp., Equisetum sp., Carex sp., Calamagrostis sp. etc., 11 Jun. 2004, A.B. Ryvkin leg. (AR); 1 o, 2 우, Tomsk Government, Ob' River between Barnaul and Chesnokovka (postal station), 13 Jun. 1897, Silant'yev leg. (ZIN); 1 ô, 1 ㅇ, Tuva, East shore of Chagytay Lake, river bank, 18 Jul. 1980, B.A. Korotyayev leg. (AR); 1 Ô, Krasnoyarsk Territory, Beryozovskiy District, 'Stolby' Nature Reserve, Mana River near Berly cordon, shingles at river bank, 22 Jun. 1990, A.B. Ryvkin leg. (AR); 1 q, Evenkia, Baykitskiy District, Central Siberian Biosphere Reserve, Stolbovaya River basin: Dulkuma River (IV), $12 \mathrm{~km}$ below Topkaya River mouth, $165 \mathrm{~m}$ a.s.l., river banks: leaf litter among Poaceae gen. sp. and Salix sp., shingles, sand, silt, drift, 1 Jul. 1990, A.B.Ryvkin leg. (AR); 1 गे, Evenkia, Baykitskiy District, Central Siberian State Reserve, Stolbovaya River basin: lower reaches of Birapchana River near mouth of Kruten'kiy Stream, $110 \mathrm{~m}$ a.s.l., river bank: shingles, silt, sand, clay, Poaceae gen. sp., mosses, Carex spp., etc., 21 Jul. 1991, A.B. Ryvkin leg. (AR); $2 \widehat{\partial} \hat{\partial}$, Krasnoyarsk Territory, Turukhanskiy District, Nizhnyaya Sarchikha River near Kamenka River mouth, 150 m a.s.l., under mosses on river bank, 6-7 Jul. 1992, V.B. Semenov leg. (AR); 1 q, same locality and collector, river bank: sand, 10 Jul. 1992 (AR); 1 ô, Krasnoyarsk Territory, Turukhanskiy District, Yeloguy River, 7-10 km below Tyna River mouth, $70 \mathrm{~m}$ a.s.1., sandy river bank with small shingles, 19 Jul. 1989, A.B. Ryvkin leg. (AR); 1 J, 1 \%, same locality and collector, river bank: Carex spp., Poaceae gen. sp., mosses, shingles, 24 Jul. 1989 (AR); 1 ð̃, same locality, Sphagnum bog with Eriophorum sp., Ledum palustre, Oxycoccus sp., Rubus chamaemorus, Vaccinium uliginosum and sparse Pinus sylvestris, 1 Jul. 1989, R.A. Rakitov leg. (AR); 1 đ̊, 3 q 9 , Krasnoyarsk Territory, Turukhanskiy District, Yeloguy Refuge, Yeloguy River $6 \mathrm{~km}$ below Tyna River mouth, river bank: under drift, 22-23 Jul. 1992, V.B. Semenov leg. (AR); 1 ô, Krasnoyarsk Territory, Turukhanskiy District, Yeloguy Refuge, Tyna River $12 \mathrm{~km}$ up-stream of river mouth, under mosses on stony bank of river, 12 Aug. 1992,

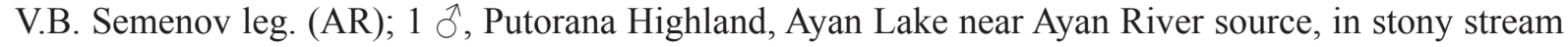
channel, 9 Jul. 1983, K.Yu. Eskov leg. (AR); 1 Ô, Taymyr, Maymecha River mouth, 5 Jul. 1971, A.P. Rasnitsyn, A.G. Ponomarenko, I.D. Sukatcheva, V.V.Zherikhin leg. (AR); 1 ठ, Taymyr, Khatangskiy District, Kotuy River, 6 km up-stream of Kresty, Salix sp., 13 Jul. 1976, A.P. Rasnitsyn \& I.D. Sukatcheva leg. (AR); 1 O, 5 웅, 'Irkutsk. V.Ye. Yakovlev leg.', 'canaliculatus Gyll. L. Benick det.' (ZIN); 1 ㅇ, 'Irkutsk. V.Ye. Yakovlev leg.', 'canal. v. cordaticollis Leinbg. L. Benick det.' <immature specimen> (ZIN); 1 ○े, 'Irkutsk. V.Ye. Yakovlev leg.', 'Coll. Semenov-Tian-Shansky' (ZIN); 1 ô, Buryatia, Selenga River, sedge community, 3 Jul. 1982, Dubeshko leg. (AR); 1 §, Buryatia, middle reaches of Vitim River, Baysa, 3 Aug. 1969, V.V. Zherikhin, Ye. Shnitnikova leg. (AR); 1 ô, [Yakutia], '5 verst off Yakutsk, suburban settlement. 18.vi.1925. Bianki leg.', 'Yakutian research expedition of Acad. of Sci.' (ZIN); 1 ㅇ, 'Yakutsk. 21.ix.1927. Moskvin leg.', '168', 'canaliculatus Gyll. L. Benick det.' (ZIN); 1 đ', '2nd Tyhylyminskiy Nasleg, Yakutsk Region. 02.vii.1925. Bianki leg.', 'Yakutian research expedition of Acad. of Sci.', 'canaliculatus Gyll. L. Benick det.' (ZIN); 1 \%, Central Yakutia, Nizhniy Bestyakh (ASh); 1 ô, W Yakutia, 11 km SW of Toybokhoy, Uchugey Syhylakh, sedge-gramineous hygrophytous meadow, 25 Aug. 1997, Stepanov leg., 'Stenus carbonarius Gyll.' (ASh); 1 + , Khabarovsk Territory, Okhotsk, Airport, wall of timber house, 4 Jul. 1985, V. Zherikhin, A. Rasnitsyn, D. Shcherbakov leg. 

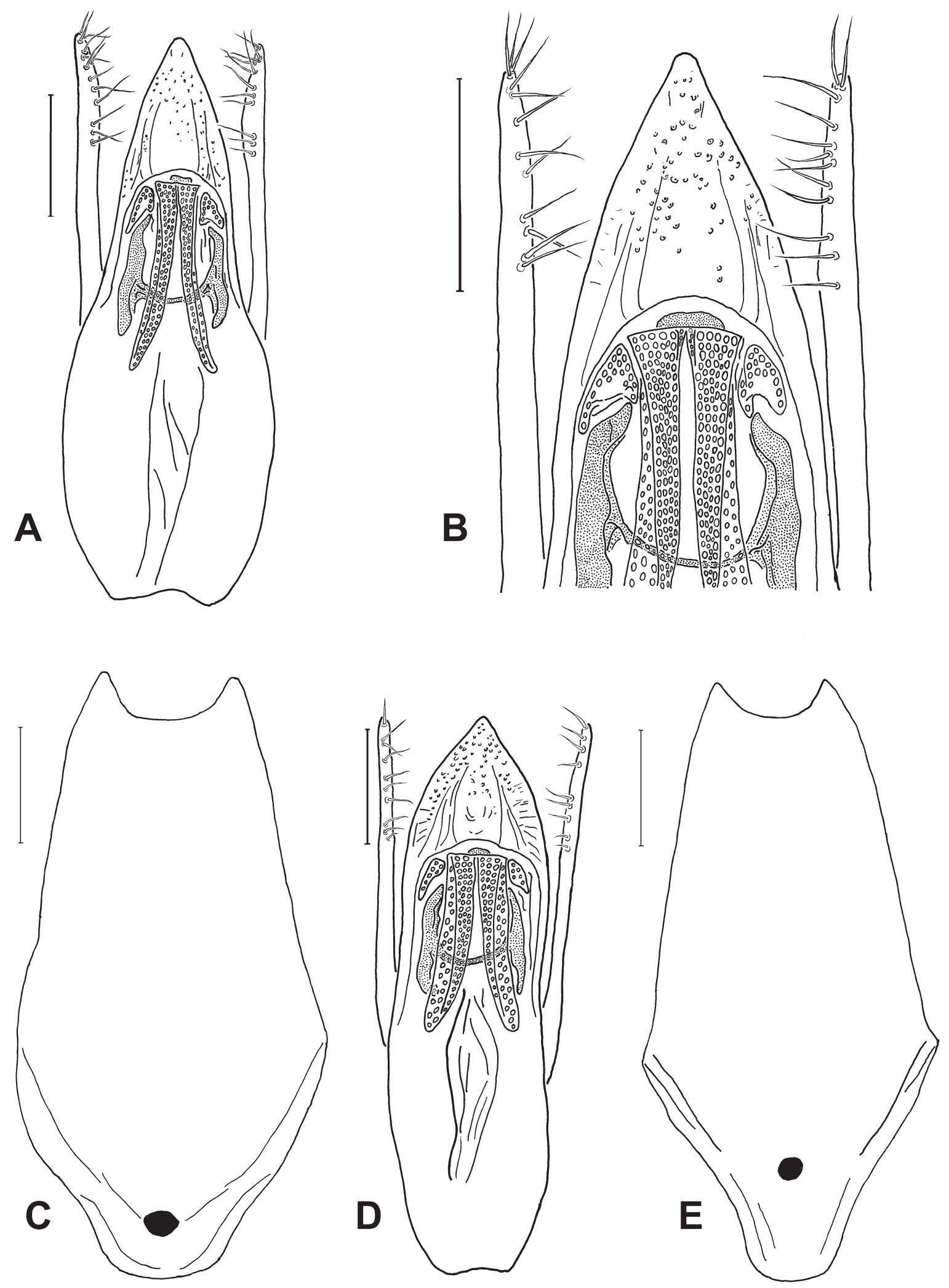

Fig. 3. - A-C: Stenus (Nestus) alopex sp. nov.: O. A. Aedeagus, ventral view (holotype). B. Apical part of aedeagus, ventral view (holotype). C. Abdominal sternite 9 (paratype). - D-E: $S$. (N.) canaliculatus Gyllenhal, 1827: $\widehat{\jmath}$. D. Aedeagus, ventral view (Russia: Rostov Area: Nedvigovka). E. Abdominal sternite 9 (Russia: Taimyr: Maymecha River). - Scales $=0.1 \mathrm{~mm}$. 
(AR); 1 , Maritime Prov., Khasan, under boards on littoral, 3 Jun. 1967, A.L. Tichomirova leg. <?> (AR).

MONGOLIA: 1 ð̊, Seleng.[a] Aimak, Shamar, 18 Jul. 1984, S.L. Kuz'min leg. (AR).

USA: 1 q, 'Wisconsin: Walworth Co.; Troy Center. (SW) Girl Scout Camp Area. W. Suter leg. 27:VI:1976.', 'FM(HD)\#76-92. WS\#76-55. mosses ex boggy field.' (FMNH); 1 §, 'USA: Ill., Kendall Co., Silver Springs St. Pk. 26-IV-1981.', 'FMHD\#81-164, ex rotting wood chips, L. Watrous leg.' (FMNH); 1 9, 'USA: Mich. Berrien Co., 2 mi. N. New Buffalo, Gallien R. W bank 2-xi-80.', 'litter in marsh. L.E. Watrous leg.' (FMNH); 1 9 , '19' <indian ink on white rectangle>, 'Kenai' $<$ indian ink on green stripe>, 'Stenus $\mid$ congener $\mid$ Mäkl.| Am. b. occ.' <indian ink on green rectangle> (ZMMU: Motschulsky collection).

\section{Remarks}

The species is variable in both external characters and, to a lesser extent, in the shape of the aedeagus. Terra typica: 'Suecia' [= Sweden]. Widespread in the Holarctic. In an earlier article devoted to the Caucasian stenine fauna, I summarized the range as follows: 'Palearctic and Alaska $<\ldots>$, Michigan $\langle\ldots\rangle$, but missing evidently in subtropics and in the southernmost districts of the Pontic biogeographical province (Turkestanian and Kurdian). Unknown to me also from the Crimean, Derbentian, Caucasian, Lenkoranian districts. The records nearest to the Caucasus are in the Rostov Area (Nedvigovka, Azhinov, Manychskaya, Yerokhin, Podtyolkovo, Liventsovka). One cannot deny a possibility of finding it in Ciscaucasia' (Ryvkin 1990; the biogeographical units follow those of Razumovskiy 1980). In this excerpt I missed the important record from NE Turkey: 'Benliahmed südwestl. Kars' (Korge 1971); though the locality does not belong to the Caucasus in a strict sense, it lies right at the border between the Caucasian and Kurdian biogeographical districts. This species is missing in the lists for the NW Caucasus (Solodovnikov 1998) and the Samara Area (Goreslavets et al. 2002), but my restudy of the collections of the named authors makes it possible to report $S$. canaliculatus for both the regions (see above: the Material section).

Both records of S. canaliculatus in Shavrin (1998) (for "Alar', Irkutsk Area", and "Bol'shoye Alginskoye Lake, Buryatia") are to be referred to S. incrassatus Erichson, 1839 (I have seen the material in Shavrin's collection). Among the material listed under the name 'canaliculatus' by Shavrin \& Puthz (2007), at least the specimen from 'Barun-Torej, Myrgen', Chita Area should be referred to the new species S. canalis sp. nov. described below; some other Transbaikalian specimens unknown to me may also prove to belong to this new species. In that article, all the records marked as relating to the material deposited in 'coll. A.Shavrin' and 'Irkutsk State University', except 'Sarma', 'Shelekhov', and 'Selenga delta' (the latter locality, based on my identifications, has been published by Dubeshko (1984)), should be verified because the respective specimens were not present in the complete collection that Dr. Shavrin has sent me for restudy.

Ryabukhin (1999), based on my identifications in the IBPM collection, listed three localities from Kamchatka.

Campbell \& Davies (1991) reported this species for Alaska and all the provinces of Canada, excluding Saskatchewan, Labrador, and Prince Edward Island, without citing material. The American specimens I know from the northernmost localities are very similar to those from NE Asia; but the material from the USA main territory as well as from S Canada demonstrates definite differences both in body proportions (e.g. the head somewhat more wide) and in the shape of the male genitalia; I believe the variability mentioned above requires further analysis based on more representative sampling. 
S. congener Maeklin, 1853, according to the original description, was described for a single specimen captured on Kadjak Island ('In insula Kadjak a D.Holmberg semel captus'). No specimens from the Kenai Peninsula were mentioned in Maeklin's text; these two localities were distinguished well by both Mäklin and Mannerheim (a good map was provided in Mannerheim 1853, which incorporated Maeklin's descriptions); therefore, assumptions by Silfverberg (1988) and Puthz (2001) concerning the type status of Kenai specimens in different museums seem to be as premature as unfounded ('semel captus' $<$ ! $>$ means that the type series cannot be distributed widely over all the main European museums). Casey (1884), L. Benick (1921b), and Scheerpeltz (1933) treated S. congener as a species propria; nevertheless, Fauvel (1869, 1873, 1889), Rey (1884), Bernhauer \& Shubert (1911), and others regarded this name as a synonym of $S$. canaliculatus. I think it important that Fauvel (1889), when citing the synonymy, mentioned Maeklin's type as known to him and cited the terra typica as 'Kadjak'. Consequently, the synonymy of congener and canaliculatus should be regarded as hypothetical before a reliable type specimen can be found.

\section{Stenus (Nestus) alopex sp. nov.}

(Fig. 3A-C)

\section{Material examined}

RUSSIA: Holotype: $\hat{\partial}$, 'Putorana Highland, Ayan Lake near | source of Ayan River. | Lakeshore osier | tundroid. 22.08.1983. | K.Yu. Eskov. \#74.' < printed label in Russian>, 'HOLOTYPUS' <my standard printed red label>, 'Stenus HT | alopex sp. n. | A.B. Ryvkin det., 1994'<my standard determinative

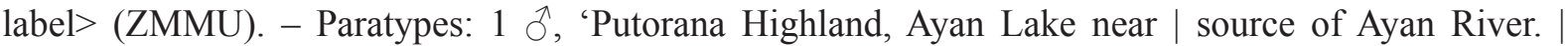
Lakeshore tundroid. | 23.08.1983. | K.Yu. Eskov. \#75.' < printed label in Russian> (AR); 1 \&, 'Putorana Highland, Ayan Lake. | Alpine belt. | 28.07.1983. | K. Yu. Eskov. \#54.' <printed label in Russian> (AR);

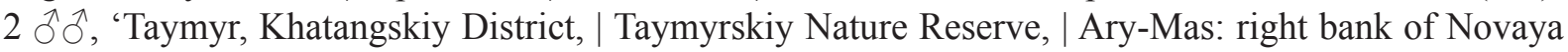
River | near field research station. [Mosses and litter under dwarf Salix sp. and Betula ?exilis] near small rill. | 17.07.1992. A. Ryvkin. \#93.' < printed label in Russian> (IRSN, MTD); 1 ô, 1 क , 'Taymyr, Khatangskiy District, | Taymyrskiy Nature Reserve, | Ary-Mas: right side of Novaya River | near Ulakhan-Yuryakh River mouth. Bank | of [Novaya] River[: shingles, sand]. 19.07.1992. A. Ryvkin. \#98.' < printed label in Russian> (AR); 2 우, 'Taymyr, Khatangskiy District, | Taymyrskiy Nature Reserve, | Ary-Mas: right side of Novaya River | near Ulakhan-Yuryakh River mouth. | Swampy floodplain of [small] rill[: mosses and litter among Carex spp., Salix spp., Betula ?exilis etc.]. | 19.07.1992, A. Ryvkin. \#99.' < printed label in Russian> (AR); 1 q, 'Taymyr, Khatangskiy District, | Taymyrskiy Nature Reserve, | Ary-Mas: right bank of Novaya River | near field research station. Shore of 'small' | lake [ESE of Ulakhan-Yuryakh River mouth. Mosses, litter and sweeping at lake shore: Carex spp., Comarum palustre]. 20.07.1992. A. Ryvkin. \#104.' <printed label in Russian> (AR); $2 \lesssim ð$, 'Taymyr, Khatangskiy District, | Taymyrskiy Nature Reserve, | Ary-Mas: right bank | of Novaya River, near lake between | small rill and Ulakhan- | Yuryakh River.', 'Mosses and litter under Larix [gmelinii] with | Cassiope tetragona, Pyrola | sp., Vaccinium vitis-idaea etc. | on S slope. 23.07.1992. | A.B. Ryvkin. \#115.' < printed labels in Russian $><$ one specimen without head and pronotum $>$ (AR); 1 o , 1 + , 'Taymyr, Khatangskiy District, | Taymyr Nature Reserve, | Ary-Mas: right bank | of Novaya River near mouth of Ulakhan- | Yuryakh River (watershed of Novaya River and | small rill).', 'Mosses and litter on glade | in sparse larch forest | with Betula ?exilis, Salix spp., | Vaccinium vitis-idaea, V. | uliginosum, Pyrola sp., | Cassiope tetragona, Cladonia | spp., Carex spp., etc.| 24.07.1992. A.B. Ryvkin. \#118.' < printed labels in Russian> (AR). - The paratypes listed above are also provided with both "PARATYPUS" printed red label and my standard determinative label ('Stenus | alopex sp. nov. | A.B.Ryvkin det., 1994') for each lot. - 1 +, Chukot Peninsula, 78-84, Ayopechan Island, Ust'-Chaun, 19 Jul. 1978, E.G. Matis leg. $<?>$ (AR). 


\section{Differential diagnosis}

This species resembles $S$. (N.) canaliculatus Gyllenhal, 1827 and can be distinguished from it by the narrower and shorter elytra, by the much elongated pronotum, by the character of puncturation, and by the shape of the aedeagus. External differences from all the known species of the canaliculatus-group are given in the key below.

\section{Etymology}

The specific name is derived from the Latin generic name of the arctic fox Alopex lagopus (Linnaeus, 1758).

\section{Description}

LENGTH. 3.2-3.9 $\mathrm{mm}$ (the last value for the specimen with abdomen extended).

COLORATION. Black, moderately shining, with moderately long and dense greyish- or yellowish-silvery pubescence. Legs pitchy black, tibial apices and tarsi somewhat lighter. Antennae pitchy brown, with segments 1-2 black and club vaguely infuscate; 1st segment of maxillar palpi yellow, 2nd brown with yellowish base, 3rd dark brown, with base a bit lighter.

HEAD. About $1 / 5$ to $1 / 6$ broader than pronotum (47:39), exactly as broad as elytra between humeri (47:47), distinctly narrower than those in broadest part (47:54). Front feebly evenly convex, with very vague and shallow lateral impressions distinguishable only between antennal tubercles and eyes. Puncturation moderately dense and regular, nonconfluent, as a rule, except of periocular areas, without smooth spots and strips; average diameter of punctures about as large as basal cross-section of antennal segment 3. Frontal slope before antennal fossae rather flat. Anterior margin of labrum with a small median notch. Antennae moderately long, reaching the middle 1/3 of pronotum. Length proportions of antennal segments $2-11=6: 5: 4.5: 5: 4: 4: 3: 3.5: 4.5: 4$; segments of club a little longer than broad (3.5:3, $4.5: 4,4: 3.5)$.

Pronotum. Evidently longer than broad (45:39), broadest near middle, narrowed feebly convexly towards anterior margin and feebly concavely towards posterior one. Longitudinal median furrow not deep but evident over almost entire length of disk. Laterobasal depressions very shallow, nearly vanishing. Puncturation dense and fairly deep, sometimes nonrugosely confluent near midline; punctures somewhat larger than those of head.

ELYTRA. A bit broader than long (54:52), distinctly longer than pronotum (52:45 in holotype), at suture nearly as long as the latter (46:45 in holotype). Humeral angles short but evident, lateral sides uniformly moderately divergent posteriorly (47:54). Humeral and sutural depressions nearly absent. Puncturation deep and fairly regular, evidently larger in diameter than that of pronotal disk, sometimes confluent, but not forming long furrows.

LEGS. Fairly short; segment 1 of metatarsi about as long as segment 5.

АвDomen. Moderately convex, with well developed paratergites and two pairs of keels at bases of anterior visible tergites. Lateral sides nearly parallel, feebly convergent in apical part. Posterior margin of tergite 7 with fine light fringe. Puncturation of tergites much smaller than that of head and pronotum, on anterior visible tergites denser laterally, medioposteriorly with some interstices comparable to diameter of punctures.

Microsculpture. The whole dorsal side with fine and dense ground sculpture visible throughout. 
MALE. Meso- and metatibiae without specific characters; posterior margin of 8th abdominal sternite with a fairly broad but not deep angularly-rounded emargination, without deep impressions and keels, slightly depressed in posterior half; abdominal sternite 9 as in Fig. 3C; aedeagus as in Fig. 3A, B.

FeMALE. Posterior margin of 8th abdominal sternite angularly rounded.

\section{Remarks}

This new species seems to be a late derivative of $S$. (N.) canaliculatus Gyllenhal, 1827; the latter also inhabits both the Taymyr Peninsula and the Putorana Highland (see above). Additional material including male specimens is necessary to prove the distribution of the new species up to the Chukot Peninsula.

\section{Stenus (Nestus) canosus sp. nov.}

(Figs. 4A-B, 5D)

\section{Material examined}

CHINA: Holotype: $\hat{O}$, 'NW China, Xinjiang Uygur | Zizhiqu, Tien | Shan, Southern slope of Narat Mt Ridge: | Koktau Mts, right confluent | of Koksu River, 3200 m, stone-| sandy bank of rill with sedge tussocks and $\mid$ small willows. 25.07.[20]01. [S.V.]Saluk. $\mathrm{N}^{\circ} 11^{\prime}<$ printed label in Russian>, 'HOLOTYPUS' < my standard printed red label>, 'Stenus HT | canosus sp. n. | A.B.Ryvkin det., 2010' $<$ my standard determinative label $>$ (ZMMU).

\section{Differential diagnosis}

This new species is externally very similar to $S$. (N.) canaliculatus Gyllenhal, 1827 and differs from it by the character of the puncturation, which is much coarser, more sparse and irregular, by the less developed microsculpture, by the median furrow of pronotum, which is deeper and broader, and by the shape of the aedeagus. External differences from all the known species of the canaliculatus-group are given in the key below.

\section{Etymology}

The name of this species is the Latin adjective "canosus" (grey-haired).

\section{Description}

LENGTH. $4.0 \mathrm{~mm}$ (with abdomen extended).

Coloration. Black, distinctly shining, with moderately long and dense silvery pubescence. Legs dark brown with knees somewhat darkened, brownish-black. Antennae dark brown, with segments 1-2 pitchy-black and club vaguely infuscate; 1st segment of maxillar palpi yellow, 2nd brown with yellow base, 3rd dark brown with very base yellowish.

HEAD. Distinctly broader than pronotum (50:44), somewhat narrower than elytra between humeri (50:53), much narrower than those in their broadest part (47:54). Front feebly evenly convex, with very vague and shallow lateral impressions distinguishable mainly between antennal tubercles and eyes. Puncturation moderately dense, somewhat irregular, evidently smaller and denser between antennal tubercles, partly nonrugosely confluent both there and at periocular areas, without distinct smooth spots and strips, but obviously sparser and greater in the middle; average diameter of punctures in the middle distinctly larger than the greatest cross-section of antennal segment 3. Frontal slope before antennal fossae rather flat. Anterior margin of labrum with small median notch. Antennae moderately long, scarcely reaching middle of pronotum. Length proportions of antennal segments $2-11=6: 5: 6: 4: 4: 4: 3: 4: 4.5: 6$; segments of club longer than broad (4:3, 4.5:4, 6:4). 

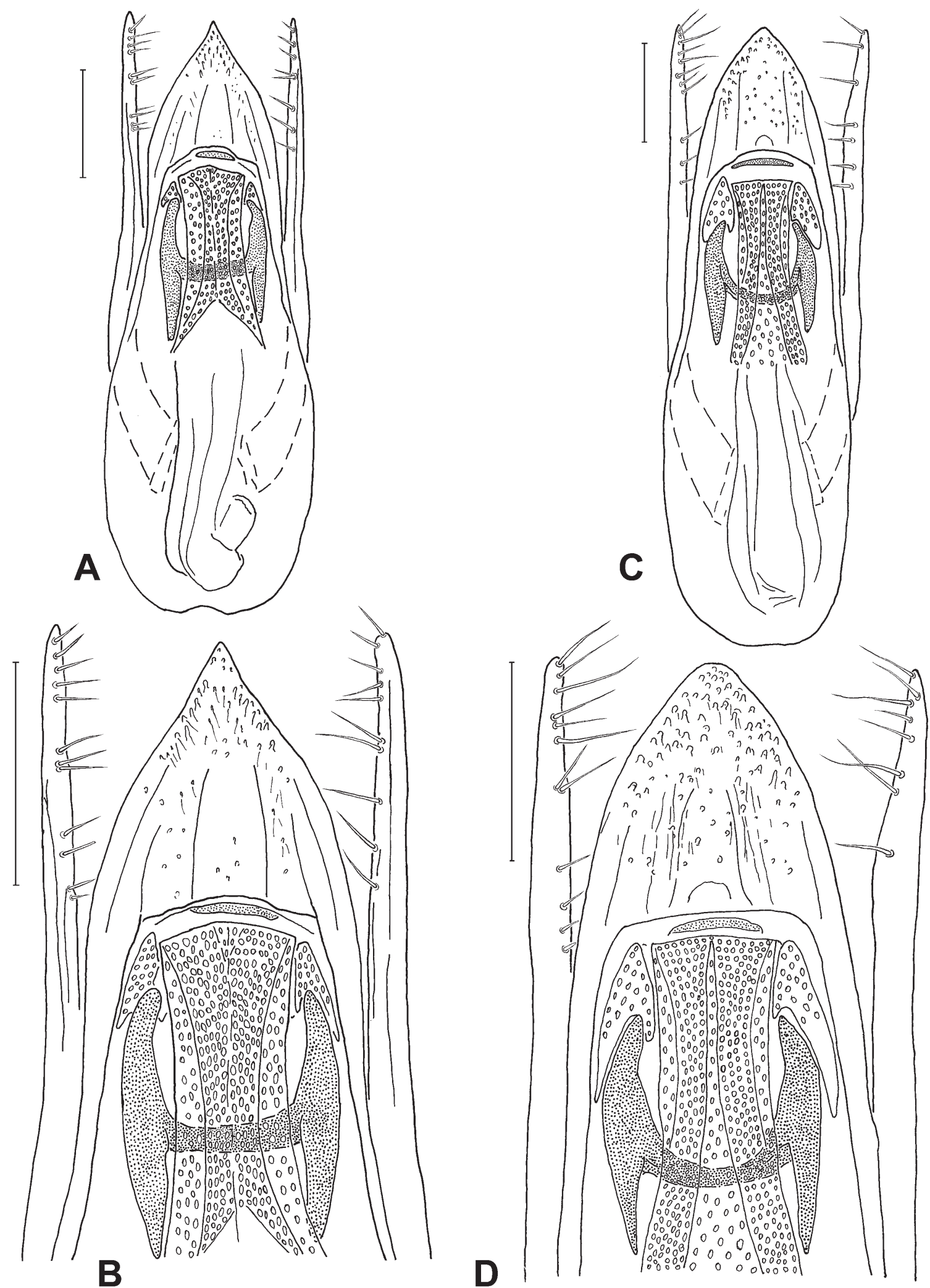

Fig. 4. - A-B: Stenus (Nestus) canosus sp. nov.: $\widehat{o}$ (holotype). A. Aedeagus, ventral view. B. Apical part of aedeagus, ventral view. - C-D: $S$. (N.) canalis sp. nov.: $\widehat{o}$ (holotype). C. Aedeagus, ventral view. D. Apical part of aedeagus, ventral view. [Note that the difference in shape of the apical lobe compared to that in $\mathrm{C}$ is due to the level of magnification.] - Scales $=0.1 \mathrm{~mm}$. 
Pronotum. A bit longer than broad (45:44), broadest near middle of length, narrowed feebly convexly towards anterior margin and concavely towards posterior one. Longitudinal median furrow deep and sharp over entire length except for very anterior piece. Laterobasal depressions very shallow, nearly vanishing. Puncturation deep and irregular, partly nonrugosely confluent; punctures distinctly larger than those of head.

ELYTRA. A bit broader than long (60:58), much longer than pronotum (58:45), at suture distinctly longer than the latter (48:45). Humeri anglarly rounded, lateral sides uniformly moderately divergent posteriorly (53:60). Humeral and sutural depressions feeble but evident in anterior half of disk. Puncturation deep, much more regular, evidently larger in diameter than that of head and pronotum, sometimes confluent, but not forming long furrows.

Legs. Fairly short; metatibia about 1/3 longer than metatarsus (40:29); segment 1 of metatarsi about as long as segment $5(8: 8)$.

ABDOMEN. Moderately convex, with well developed paratergites and two pairs of keels at bases of anterior visible tergites. Lateral sides feebly uniformly convergent. Posterior margin of tergite 7 with fine light fringe. Puncturation of tergites evidently smaller than that of head and pronotum; on anterior visible tergites much denser and smaller laterally, medioposteriorly with some smooth interstices distinctly wider than average diameter of punctures.

Microsculpture. Very fine mesh-like ground sculpture visible between punctures, mainly on elytra and abdominal tergites $8-9$, as well as on lateral parts of head, pronotum and anterior abdominal tergites, median parts of abdominal tergites quite smooth or slightly irregularly netted only on edges of punctures.

MALE. Meso- and metatibiae without specific characters; 8th abdominal sternite without conspicuous impressions and keels, its posterior margin with very broad and not deep angularly-rounded emargination; abdominal sternite 9 as in Fig. 5D; aedeagus as in Fig. 4A, B.

Female. Unknown.

\section{Remarks}

No records of $S$. (N.) canaliculatus were previously known from China or from adjacent countries of Central Asia. The capture of $S$. (N.) canosus sp. nov., which is closely related to $S$. (N.) canaliculatus, proves that their common ancestor was recently widely distributed throughout these spacious territories.

Stenus (Nestus) canalis sp. nov.

(Figs. 4C-D, 5E)

Stenus canaliculatus - Shavrin \& Puthz 2007: 114 (pars).

\section{Material examined}

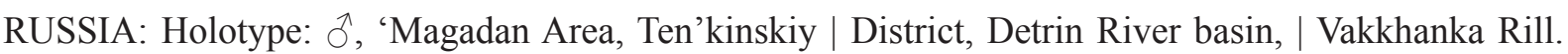
Plateau: open | plots, soil traps. | 01-11.06.1983. S.P. Bukhkalo [leg.]' <printed label in Russian>, 'HOLOTYPUS' < my standard printed red label>, 'Stenus HT | canalis sp. n. | A.B. Ryvkin det., 2010' $<$ my standard determinative label> (ZMMU). Paratypes: 10 , 'Magadan Area, Ten'kinskiy | District, near Sibit-Tyhellakh. | Dry birch forest. 14-24.07.1982. | D.I. Berman [leg.]' <printed label in Russian> (AR); 1 o, 'Khabarovsk Territory, Jewish | Autonomous Area, Obluchenskiy | District, near Pashkovo. Puddle | bank under steep. | 05.07.1977. A.B. Ryvkin [leg.]' <printed label in Russian>, 'Stenus | 
canaliculatus Gyll. |A.B. Ryvkin det. 1978' (AR); 1 ภ̂, 'Maritime Province, Khasan, | Golubinyi Utyos. | 4.viii. | 1991 S.A. Kurbatov [leg.]' <photo label in Russian> (AR); 1 ô, 'Maritime Prov. | flood-plain of Poyma River | 30.07.-06.08.[19]99 | Shavrin A. [leg.]' <printed label in Russian>, 'Stenus Gyll. | canaliculatus | det. V. Puthz 2005' (AR); 1 §, 'Chita Area | NE side of Barun-Torey Lake | Myrgen Cape | 18-19.06.1994 | V.[G.] Shilenkov leg.' <printed label in Russian>, 'Stenus Gyll. | canaliculatus | det. V. Puthz 1999' (AR); 1 o, '5.08.[19]86 < transverse > | Buryatya $\mid$ Zaigraevo[=Zaigrayevo] 1695 [=5695th km] st[ation] | Voincov[=Voinkov] A. [leg.]' <printed label>, '3-23 | canal.', 'Stenus | canaliculatus | Shavrin A. det. 2006' (ASh). - The paratypes listed above are also provided with both 'PARATYPUS' printed red label and my standard determinative label ('Stenus $\mid$ canalis sp. n. |A.B. Ryvkin det., 2010') for each lot. - 1 , Magadan Area, upper reaches of Kolyma River, $7 \mathrm{~km}$ NNE of Omsukchan, $10 \mathrm{Jul}$. 1980, O. Mashukova leg. <?> (AR); 1 , Magadan Area, upper reaches of Kolyma River, 5 km NE of Spornoye, 21 Jun. 1981, A. Ryabukhin leg. <?> (AR).

\section{Differential diagnosis}

This species is closely related to $S$. (N.) canaliculatus Gyllenhal, 1827 and differs from it by the smaller body size, by the much more irregular puncturation, by the less developed ground sculpture, by the posterior margin of 8th abdominal sternite, which is much more shallowly emarginated, and by the shape of the aedeagus. External differences from all the known species of the canaliculatus-group are given in the key below.

\section{Etymology}

The specific name is the Latin adjective 'canalis' (canine).

\section{Description}

LENGTH. 2.8-3.7 $\mathrm{mm}$ (the latter value for the paratype specimen with abdomen extended).

Coloration. Pitchy black, distinctly bronze-coloured shining, with moderately long and dense yellowish-silvery pubescence. Legs pitchy black to brownish-black, tibial apices and tarsi somewhat lighter. Antennae dark brown, with $1^{\text {st }}$ segment black and $2^{\text {nd }}$ brownish-black; $1^{\text {st }}$ segment of maxillar palpi yellow, $2^{\text {nd }}$ brown, 3rd dark brown, with very base a bit lighter.

HEAD. About $1 / 8$ to $1 / 6$ broader than pronotum (46:41, all measurements, except where specifically indicated, are from the holotype), a little narrower than elytra between humeri (46:48), distinctly narrower than elytra in broadest part (46:55). Front feebly evenly convex to nearly flat, with very vague and shallow lateral depressions, distinguishable only between antennal tubercles and eyes and behind the tubercles. Puncturation dense and irregular, partly nonrugosely confluent, sometimes with small but evident smooth spots and strips situated both medially and laterally; average diameter of punctures in middle about as large as the greatest cross-section of antennal segment 3. Frontal slope before antennal fossae rather flat. Anterior margin of labrum with a small median notch. Antennae rather short, scarcely reaching middle $1 / 3$ of pronotum. Length proportions of antennal segments $2-11=6: 5: 5: 4: 3: 3: 2: 3: 4: 6$; segments 9-10 slightly transverse $(3: 3.5,4: 4.5)$, the segment 11 distinctly elongate $(6: 4)$.

Pronotum. Somewhat longer than broad (45:41), broadest just before middle, narrowed feebly convexly towards anterior margin and concavely towards posterior one. Longitudinal median furrow sharp and deep, well-developed over entire length of disk as a rule, but less evident in some paratypes in anterior $1 / 3$ as well as near both anterior and posterior margins. Laterobasal depressions very shallow, nearly vanishing. Puncturation rather deep and irregular, partly nonrugosely confluent but not forming long furrows; punctures somewhat larger on average than those of head; some bulging smooth interstices between punctures evidently smaller in diameter than surrounding punctures. 
Elytra. About as broad as long (55:55) to a bit broader, much longer than pronotum (55:45), by suture a bit longer than pronotum (46:45). Humeral angles short, angularly rounded, lateral sides uniformly moderately divergent posteriorly (48:55). Humeral and sutural depressions very feeble to nearly absent. Puncturation larger in diameter and sparser, more regular than that of pronotal disk, as a rule nonconfluent, but individual furrows of merged punctures visible in some paratypes; interstices between punctures distinctly smaller than half average diameter of puncture.

Legs. Fairly short; metatibia much longer than metatarsus (38:27 in paratype from Magadan Area); segment 1 of metatarsi about as long as segment 5 (7:7 in the same paratype).

ABDomen. Moderately convex, with well developed paratergites and two pairs of keels at bases of anterior visible tergites. Lateral sides of abdomen nearly parallel, feebly convergent in apical part. Posterior margin of tergite 7 with fine light fringe. Puncturation of anterior visible tergites comparable in average diameter of punctures to that of head but not so coarse and much more regular; denser laterally, medioposteriorly with some interstices comparable to diameter of punctures or larger.

Microsculpture. Variable in different specimens. Very fine mesh-like ground sculpture visible, as a rule, between punctures or by edges of punctures on elytra in humeral impressions and near suture, as well as on periocular parts of head; abdominal tergites quite smooth and glossy at least in their median parts.

MALE. Meso- and metatibiae without specific characters; $8^{\text {th }}$ abdominal sternite without conspicuous impressions and keels, its posterior margin with a very broad and shallow rounded emargination; abdominal sternite 9 as in Fig. 5E; aedeagus as in Fig. 4C, D.

Female. No reliable female specimens included in type series.

\section{Remarks}

Both the females listed were captured at localities from which no male material is known. Therefore, they are not included in the type series and their attribution to this new species remains questionable. This new species seems to replace $S$. (N.) canaliculatus Gyllenhal, 1827 in the southernmost and, in part, easternmost territories of E Siberia and the Far East. Additional material is necessary to ascertain the precise distribution of both species in the E Palaearctic.

\section{Stenus (Nestus) delitor sp. nov.}

(Figs. 5C, 6A, 7A)

\section{Material examined}

RUSSIA: Holotype: $\widehat{\partial}$, 'Evenkia, [Baykitskiy District,] basin of Podka- $\mid$ mennaya Tunguska River: Ko- | chumdek River 8-10 km up-stream of mouth |. $70 \mathrm{~m}$ a.s.l. [rill bank, among stones (mosses and litter). (He+Hm+Ho)]. 22-24.VII.1990. A.B. Ryvkin.' <photo label in Russian>, '262', 'HOLOTYPUS' $<$ my standard printed red label>, 'Stenus HT | delitor sp. n. | A.B. Ryvkin det., 2008' <my standard

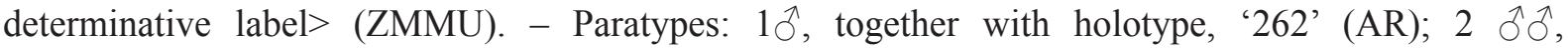
1 q, 'Evenkia, [Baykitskiy District, ] Central Sibe- | rian [Biosphere] Reserve, basin of $\mid$ Stolbovaya River: [upper reaches of ]Raskol Rill (confluent of Dulkuma River), S | slope of Dulkuma | residual mountain. 250-330 [280] m a.s.1. [Bank of rill (left confluent of Raskol Rill): litter under Alnus sp., Abies sibirica, Picea obovata and mosses near water. ]16.VII. | 1991. A.B. Ryvkin' < photo label in Russian>, '132' (AR, IRSN); 1 ○, 'Tuva, [Todjenskiy District, ]Azas Nature Reserve, basin | of Azas River: upper reaches of Kara $\mid$-Tesh River [(environs of the 3rd hut)]. $1150 \mathrm{~m}$ a.s.l. [Carex spp., mosses and Poaceae with sparse Salix spp. etc. on river bank and near small flood-plain lake.] 06.VI. | 1990. A.B. Ryvkin' 

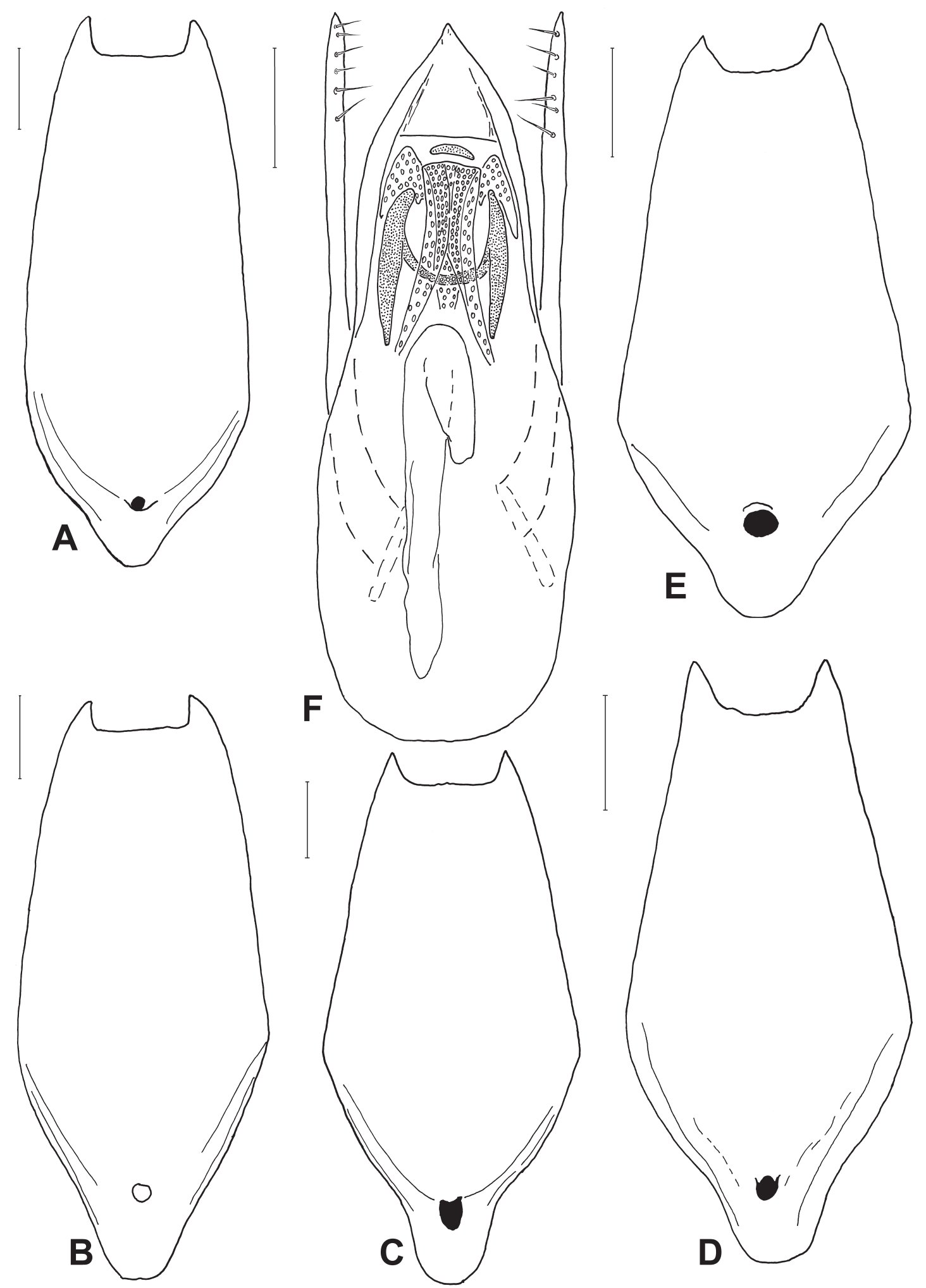

Fig. 5. ô copulative structures. A-E: Abdominal sternite 9. A. Stenus (Nestus) latipennis J. Sahlberg, 1880 (Russia: Krasnoyarsk Territory: Mirnoye). B. S. (N.) illusor Ryvkin, 1987 (Russia: Magadan Area: $2 \mathrm{~km} \mathrm{~W}$ of Splavnaya). C. S. (N.) delitor sp. nov. (holotype). D. S. (N.) canosus sp. nov. (holotype). E. S. (N.) canalis sp. nov. (holotype). F. S. (N.) caseyi Puthz, 1972 (U.S.A.: Michigan: Berrien Co.). Aedeagus, ventral view. - Scales $=0.1 \mathrm{~mm}$. 
$<$ photo label in Russian>, '64', 'Stenus $\mid$ illusor Ryv. |A.B. Ryvkin det., 1993'<my standard determinative label> (AR); $1 \hat{\delta}$, 'Khamar-Daban Mt. Ridge | pass in upper reaches | of L.[evaya] Mishikha River, $1800 \mathrm{~m}$ a.s.l. | foot of glacier | 1.VII.[19]75. Shilenkov' <photo label in Russian>, 'Stenus | latipennis | J. Sahlbg. | det. V.Puthz 1979'<standard determinative label by Puthz>, 'Stenus | illusor A. Ryv. | A.B. Ryvkin det. 1984'<my standard determinative label $><$ specimen with endophallus dissected by Puthz> (AR). - The paratypes listed above are also provided with both 'PARATYPUS' printed red label and my standard determinative label ('Stenus $\mid$ delitor sp. n. |A.B. Ryvkin det., 2008') for each lot.

\section{Differential diagnosis}

Stenus delitor sp. nov. is closely related to both $S$. (N.) latipennis J. Sahlberg, 1880 and S. (N.) illusor Ryvkin, 1987; it can be distinguished from these species by its smaller elytra, by the character of the puncturation and ground sculpture, and by the shape of the aedeagus. External differences from all the known species of the canaliculatus-group are given in the key below.

\section{Etymology}

The name of this species is the Latin noun 'delitor' (destroyer).

\section{Description}

LeNGTH. 4.0-4.6 mm (the latter value for specimens with abdomen extended).

Coloration. Black, shining, with moderately long and dense greyish-silvery pubescence. Legs black, tibial apices and tarsi sometimes a bit lighter. Anterior margin of labrum nearly sraight, without median notch. Antennae brown to dark brown, with segments 1-2 black to brownish-black; 1st segment of maxillar palpi yellow, 2nd brown with yellowish base, 3rd dark brown, with base a bit lighter.

HEAD. Much broader than pronotum (56:46), as broad as to a bit broader than elytra between humeri (56:56 in holotype), somewhat narrower than elytra in broadest part (56:62). Front with broad, moderately prominent longitudinal median ridge, obtuse-angled in cross-section, and flanked with two well developed lateral impressions; median elevation more than twice as broad as each of lateral portions in basal part (20:9). Puncturation fairly coarse and dense, irregular, partly nonrugosely confluent in twos to threes; basal part of median elevation punctured somewhat sparser but without conspicuous spots or strips impunctate; average diameter of punctures somewhat larger than basal cross-section of antennal segment 3. Frontal slope before antennal fossae fairly gentle. Antennae moderately long, scarsely reaching middle of pronotum. Length proportions of antennal segments $2-11=7: 9: 7: 7: 6: 6: 4: 5: 5: 5$; segments of club a little longer than broad $(5: 3.5,5: 4.5,5: 4)$.

Pronotum. About 1/5 to 1/6 longer than broad (54:46 in holotype), broadest near middle, narrowed convexly towards anterior margin and concavely towards posterior one. Deep median longitudinal furrow well developed over almost entire length of disk. Laterobasal depressions very shallow, though laterobasal prominences evident. Puncturation fairly coarse, irregular, somewhat greater in middle of lengh, partly transversely confluent; punctures much greater than those of head.

ElYTRA. As broad as to a bit broader than long (62:61 in holotype), distinctly longer than pronotum (61:54 in holotype), although a bit shorter than pronotum (52:54 in holotype) by suture. Humeral angles short but evident, lateral sides uniformly moderately divergent posteriorly (56:62). Humeral and sutural depressions very feeble, visible near base only. Puncturation deep, more regular and evidently larger in average diameter than that of pronotum, partly confluent, but not forming long furrows.

LEGS. Moderately long; metatibia nearly $1 / 2$ longer than metatarsus (54:37); 1 st segment of metatarsi a bit longer than $5^{\text {th }}$ segment $(11: 10.5)$. 
ABDOMEN. Moderately convex, with well developed paratergites and two pairs of keels at bases of anterior visible tergites. Lateral sides of abdomen nearly parallel, feebly convergent in apical part. Tergite 7 with well developed light fringe at posterior margin. Puncturation of tergites fairly dense, on anterior visible tergites irregular, distinctly sparser medioposteriorly, on preapical tergites more scattered medially; average diameter of punctures on anterior visible tergite about equal to that of median elevation of head.

Microsculpture. Very fine and dense mesh-like ground sculpture well developed throughout but entirely absent in some areas both on head and pronotum and, mainly, on varnish shining interstices between punctures of elytra and along midline of anterior visible abdominal tergites; abdominal tergites 7-10 with evident reticulation. This character seems to be fairly variable in different populations.

MALE. Meso- and metatibiae without specific characters; abdominal sternites 6-7 slightly depressed along midline and cut medioposteriorly; 8th abdominal sternite with fairly broad but not deep, angularlyrounded emargination of posterior margin and with flat depression in posterior half; abdominal sternite 9 as in Fig. 5C; aedeagus as in Figs. 6A, 7A.

Female. Abdominal sternite 8 with broad, rounded apical margin.

\section{Remarks}

Until recently I have interpreted this species as being a western form of S. illusor Ryvkin, 1987 . The specimen from the Khamar-Daban was not included in the type series of the latter owing to evident differences in both external appearance and the shape of the aedeagus. Study of the material from Middle Siberia and Tuva demonstrates that $S$. delitor is a valid new species.

Stenus (Nestus) illusor Ryvkin, 1987

(Figs. 5B, 7B)

Stenus (Nestus) illusor Ryvkin, 1987: 155.

\section{Material examined}

RUSSIA: 1 , Amur Area, Selemdzhinskiy District, Selemdzha River basin, Angelokit River, $52^{\circ} 53.497^{\prime} \mathrm{N} 132^{\circ} 24.543^{\prime} \mathrm{E}, 430 \mathrm{~m}$ a.s.l., mosses and litter under Salix spp. and Alnus sp. with Poaceae gen. sp., Sphagnum squarrosum, Sph. sp., Plagiomnium sp., etc. in flood-plain, 3 Jul. 2007, E.M. Veselova \& A.B. Ryvkin leg. (AR). -1 $\hat{\sigma}^{\lambda}$, Amur Area, Selemdzhinskiy District, Selemdzha River basin, Angelokit River near mouth, 52 $53.49^{\prime} \mathrm{N} 132^{\circ} 24.5^{\prime} \mathrm{E}, 420 \mathrm{~m}$ a.s.1., mosses and litter under Populus sp., Padus sp., Alnus sp., Abies nephrolepis, Picea ajanensis, etc. with Spiraea spp., Swida alba, Rosa sp., Rhododendron sp., sparse Betula platyphylla, Poaceae gen. spp., Carex spp., Smilacina davurica, Pyrola daurica, Trientalis europaea, etc. in flood-plain forest, 4 Jul. 2007, E.M. Veselova \& A.B. Ryvkin leg. (AR); 1 क , Amur Area, Selemdzhinskiy District, left side of Selemdzha River, 3 km below Butov Kamen' rapid, gorge with waterfall, $52^{\circ} 50.94^{\prime} \mathrm{N} 132^{\circ} 11.44^{\prime} \mathrm{E}, 400 \mathrm{~m}$ a.s.1., mosses and litter on stones and rocks near, above and below the waterfall, 8 Jul. 2007, E.M. Veselova \& A.B. Ryvkin leg. (AR). $-1 \hat{\jmath}$, Khabarovsk Territory, Verkhnebureinskiy District, island on Niman River $700 \mathrm{~m}$ upstream of Niman cordon of Bureinskiy Nature Reserve, $1040 \mathrm{~m}$ a.s.l., mosses and leaf litter in floodplain forest with Populus sp., Salix sp., Spiraea spp., Sorbaria sorbifolia, Poaceae gen. spp., Carex spp., Sphagnum sp., Polytrichum sp., Hylocomium splendens, etc., 11 Aug. 2008, A.B. Ryvkin leg. (AR); 1 ô, 1 q, Magadan Area, 2 km W of Splavnaya, №236, 2 Sep. 1981, A. Ryabukhin leg. (AR); 1 , Magadan Area, $35 \mathrm{~km}$ N of Magadan, №184, 16 Aug. 1981, A. Ryabukhin leg. (AR); 1 +, same locality, №194, 20 Aug. 1981, A. Ryabukhin leg. (AR); 1 , , Kamchatka, Esso, flood-plain of Bystraya River, 27 Jun. 
1975, B.A. Korotyayev leg. (AR); 1 + , Chukot Peninsula, Anadyr River basin, Novyi Yeropol, middle flood-plain, osier-bed with Poaceae on silty clay sand: soil trap, 15 Jul. 1986, E.G. Matis leg. (AR).

\section{Remarks}

The species was previously known only for the male holotype: 'Khabarovsk Territory, Amur basin, Lyanchli River, confluent of Gorin River, 5.ix.1975, O.N. Kabakov leg.' (ZIN). Based on the new material, I regard it as being widely distributed across the Russian Far East, except the southernmost territories.

\section{Stenus (Nestus) latipennis J. Sahlberg, 1880}

(Figs. 5A, 7C-H)

Stenus latipennis J. Sahlberg, 1880: 78.

Stenus latipennis - Heyden 1881: 78. - Puthz 1972d: 107. — Puthz 1974: 112. — Shavrin \& Puthz 2007: 123 (pars).

Stenus latipennis $<$ ? $>-$ J. Sahlberg 1899: 340.

Stenus (Nestus) latipennis - Jakobson 1909: 481. — Palm 1961: 98. — Puthz 1965: 27 (pars). — Puthz 1967a: 49. — Tichomirova 1973: 173. — Shilov 1975: 58. — Ryvkin 1987: 159.

Stenus (Nestus) latipennis<?>- Poppius 1909b: 19.

Stenus (s. str.) latipennis - Campbell \& Davies 1991: 111.

\section{Material examined}

RUSSIA: 1 , South of the Yamal Peninsula, Priuralskiy District, Tarcheda-Yakha River near mouth, 23 Jul. 1980, E.M. Veselova leg. (AR); 1 , $70^{\circ} 04^{\prime} \mathrm{N} 87^{\circ} 36^{\prime} \mathrm{E}$, SW Taimyr, upper reaches of Nizhnyaya Agapa River, Nyapan Ridge, at shore of Ladannakh Lake, 11-16 Jul. 2001, A.B. Babenko leg. (AR); $1 \hat{\delta}$, same locality and collector, snowfield, 5-9 Jul. 2001 (AR); $7 \hat{\delta} \delta^{\lambda}, 7$ $q$, , Taymyr, Maymecha River mouth, Yantardakh Mt, 7 Jul. 1971, A.P. Rasnitsyn, A.G. Ponomarenko, I.D. Sukatcheva,

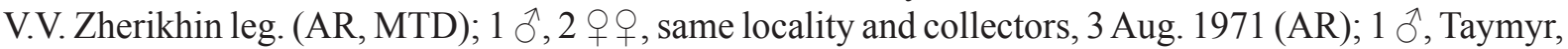
Khatangskiy District, Kotuy River, 6 km up-stream of Kresty, Salix sp., 11 Jul. 1976, A.P. Rasnitsyn \& I.D. Sukatcheva leg. (AR); 1 ô, Taymyr, Kotuy River 6 km up-stream of mouth, 18 Aug. 1971, A.P. Rasnitsyn, A.G. Ponomarenko, I.D. Sukatcheva, V.V. Zherikhin leg. (AR); 1 ô, Taymyr, Khatangskiy District, Bol'shaya Romanikha River mouth, swampy old channel, 18 Jul. 1977, I.D. Sukatcheva leg. (AR); 1 + , Taymyr, near Khatanga Town, flood-plain Salix bushes with Larix, soil traps, 16-30 Aug. 1989, P.K. Yeryomin leg. (AR); 1 đ̂, Putorana Highland, Ayan Lake near Ayan River source, mossy coastal osier-bed, 7 Jul. 1983, K.Yu. Eskov leg. (AR); 1 đ̃, same locality and collector, in stony stream channel, 9 Jul. 1983 (AR); 1 Õ, Krasnoyarsk Territory, Turukhanskiy District, Bol'shaya Varlamovka River 2-6 km up-stream of mouth, $40 \mathrm{~m}$ a.s.l., river banks, 12 Jul. 1988, A.B. Ryvkin leg. (AR); 1 đ̃, 6 우, Krasnoyarsk Territory, Turukhanskiy District, Mirnoye, traps, plate 11, 6-16 Jul. 1991, L.B. Rybalov leg. (AR, MHNG, ShIN); 1 \&, same locality and collector, trap 10, 2-12 Aug. 1990

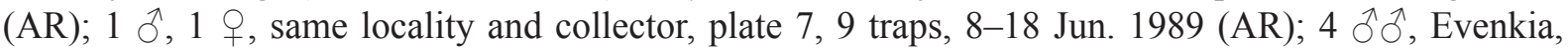
Central Siberian Biosphere Reserve, Stolbovaya River basin: lower reaches of Birapchana River near Kruten'kiy Stream, 110 m a.s.l., river bank: shingles (limestone), 23 Jun. 1993, V.B. Semenov leg. (AR); 1 गे, East Siberia, Magadan Area, Ten'kinskiy Distr., 'Aborigen' field research station, $500 \mathrm{~m}$, pebble river banks, 28 Jul.-5 Aug. 1990, L. Penev leg. (AR); 1 9, Magadan Area, 15 km SSE of Atka, 29 Jun. 1981, A.S. Ryabukhin leg. (AR); 1 Ĵ, Magadan Area, near Palatka, Khasyn, 27 Aug. 1978, V.V. Zherikhin leg. (AR). 


\section{Remarks}

The type series was collected by Nordenskjöld's Yenisei expedition at five localities from Chyornyi Ostrov ('Tschornaya ostrov' in the original description, present-day Chernoostrovsk village) to Dudinka ('Habitat ad ripas rivulorum torrentium inter lapillos in territorio frigido et arctico et parte boreali territorii silvosi rarius'). This species was reported for the first time for NE European Russia ('Petschora', without more precise locality) by J. Sahlberg (1899), based on the collection made by the botanist O. Kihlman; a decade later it was recorded from the Kanin Peninsula ('zwei Exemplare unter Moos am Rande von Schneefeldern auf dem Bergrücken (Paë) bei Bugranitza am 4.VII. und ein drittes auf gleichartigen Stellen bei Madoha am 16.VII gefunden', Poppius 1909b); Dr. Puthz has seen the two females from Kanin in $\mathrm{ZMH}$, but has not seen the specimens from Pechora (pers. comm). Jakobson (1909), not being acquainted with the latter Kanin findings, summarized the range as 'Arkhangelsk Government (Pechora); Yenisei Government'. Puthz (1965), based on earlier misidentifications by Hellén (Hansen et al. 1939; Renkonen 1941), believed the species to be represented in N Finland, notwithstanding the fact that Palm (1961) had revised all Fennoscandian records a few years earlier and noticed them to be erroneous (see $S$. (N.) labilis above and $S$. (N.) confusus below); nevertheless, Finland (without citing new material or references) was recently mentioned in the Distribution section for this species by Shavrin \& Puthz (2007); just before the present manuscript was completed, Dr. Puthz informed me that two unpublished specimens (male and female) from 'Lapponia inariensis' had been identified by him as latipennis in 1974, but that the material should be revised again (e-mail of 2 Dec. 2010). The figure of the aedeagus of the male from Dudinka (Puthz 1965) is more or less adequate, except the missing longitudinal middle keel at the apical part of the median lobe. All further mentions of this species from the Palaearctic are confined to Siberia and Polar Ural: 'Polarer Ural, Gouv.[ernment] Tobolsk' (Puthz 1967a); 'Im Museum Helsinki je ein Stück von Irkutsk und Ytyk-haja' (Puthz 1972d; I believe the specimen from the former of these two localities may be related to $S$. delitor sp. nov.; the female specimen is repeatedly cited by Shavrin \& Puthz 2007); records for Komi ASSR (Puthz 1974; Shilov 1975) do not go beyond the northernmost part of the territory (Sob' River, Vorkuta, Yeletskiy, 'Polyarnyi Ural' railway station, etc). Tichomirova (1973) indicated the range as Siberian only. This species has also been reported for the Nearctic (Puthz 1974: 'Belege aus dem North West Territory, dem Yukon Territory und Alaska im Museum Ottawa und in Waschington'; Campbell \& Davies 1991: 'AK, YK, NT'), but I do not know of any reliable American specimens and cannot state whether the range of S. latipennis is really subcircumpolar or whether the Nearctic findings concern a related species. In an earlier paper (Ryvkin 1987), I regarded S. latipennis warily as a N-Siberian species; after some closely related taxa have been described, it would probably be useful to revise all the material from other territories where the species has been recorded again.

\section{Stenus (Nestus) vinnulus Casey, 1884}

Stenus vinnulus Casey, 1884: 112.

Stenus vinnulus - Fall 1926: 61. — Puthz 1972d: 107.

Stenus (Nestus) vinnulus - Puthz 1972b: 171. — Ryvkin 1987: 159. — Silfverberg 1988: $20<$ holotype of confusoides Renkonen, 1935>. — Ryabukhin 1999: 46.

Stenus (s. str.) vinnulus - Campbell \& Davies 1991: 112.

Stenus (Nestus) confusoides Renkonen, 1935: 27.

Stenus confusoides - Strand 1954: 66. — Puthz 1970a: 39.

Stenus (Nestus) confusoides - Renkonen 1936: 179. — Palm 1961: 90. — Puthz 1965: 27. — Puthz 1967a: 49. — Tichomirova 1973: 173. 


\section{Material examined}

RUSSIA: $1 \hat{\jmath}, 1$, , Karelia, White Sea, Bay of Kandalaksha, N shore of Chupa Inlet, $500 \mathrm{~m}$ NE of Nizhnyaya Polunga: Blizhneye Lake, 18-19 Jul. 2005, P. Petrov leg. (AR); 1 q, Tuva, Todjenskiy District, Azas Nature Reserve, environs of Azas Lake, Zelyonoye Lake, 980 m a.s.l., mossy swamps with Ledum palustre, Eriophorum sp., Carex spp., Rubus chamaemorus, Rhododendron sp. etc. near banks of rill (rhadon!) - in moss, 3 Jun. 1990, A.B. Ryvkin leg. (AR); 1 +, Evenkia, Baykitskiy District, Central Siberian Biosphere Reserve, Stolbovaya River $8 \mathrm{~km}$ up-stream of river mouth, $60 \mathrm{~m}$ a.s.l., mosses and litter on open swamp with Carex spp., Comarum palustre, sparse Menyanthes trifoliata, true mosses, Sphagnum spp. etc., 20 Sep. 1991, A.B. Ryvkin leg. (AR); 1 , , Evenkia, Central Siberian Biosphere Reserve, Stolbovaya River basin: lower flow of Birapchana River near Kruten'kiy Stream, $110 \mathrm{~m}$ a.s.l., backwashing of limestone shingles at river bank, 29 Jun. 1993, V.B. Semenov leg. (AR); 1 ô, 1 ㅇ, Putorana Highland, nr. Ayan Lake, Kapchug River, riverside 'tundroid', bog with mosses and Eriophorum sp., 11 Jun. 1983, K.Yu. Eskov leg. (AR); 1 đ, Yakutia, Vilyui River basin, Kempendyayi River upstream of Kempendyay Village, 5 Aug. 1988, V. Blagoderov \& V. Zherikhin leg. (AR); 1 Õ, Magadan Area, $3 \mathrm{~km} \mathrm{~N}$ of Shirokoye, 7 Jul. 1974, B.A. Korotyayev leg. (AR+1ex: IBPM); 1 , Amur Area, Selemdzhinskiy District, near Fevral'sk, 268th km of Belogorsk-Fevral'sk road, Tikhiy rill, $275 \mathrm{~m}$ a.s.1., mosses and plant debris between sedge \& gramineous tussocks among Alnus sp., Salix sp., Spiraea sp. with Sphagnum squarrosum, Sph. spp., etc., 8 Oct. 2008, A.B. Ryvkin leg. (AR);

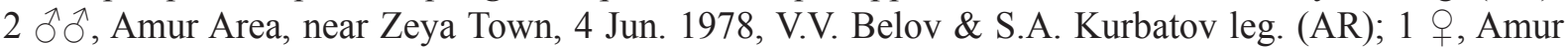
Area, near Zeya Town, Gulik, 19 Oct. 1979, S.A. Serbenyuk leg. (AR); 1 ㅇ, Amur Area, Selemdzhinskiy District, Norskiy Nature Reserve, Nora River basin, 2 km up-stream of Gryashchinskaya Mt., mosses and plant debris on small open swamp on high flood-plain: tussocks of Calamagrostis sp. and Carex spp. with Sphagnum ? girgensohnii, Sph. squarrosum, Sph. centrale, Sph. spp., Rubus arcticus, Convallaria keiskei, Salix sp., etc., 22 Aug. 2004, A.B. Ryvkin leg. (AR); 1 +, Amur Area, Selemdzhinskiy District, Norskiy Nature Reserve, Nora River basin near Maltsevskiy cordon, E side of Maltsevskoye Lake, 210 m a.s.l., sweeping on Carex spp., Poaceae gen. spp. \& motley grass, 1 Oct. 2008, E.M. Veselova \&

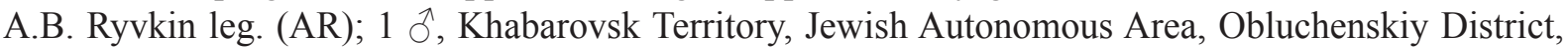
SE of Radde, Dichun River, about 2 km off river mouth, near water, 7 Aug. 1977, A.B. Ryvkin leg. (AR); 2ex, [Khabarovsk Territory,] Ussuri River basin, Bikinskiy District, Birskoye, 27 Jun. 1958, O.N. Kabakov leg. (ONK); 1 ex, same locality, 1 Jul. 1958, O.N. Kabakov leg. (ONK); 1 \&, Maritime Province, Spasskiy District, nr. Novoselskoye, rice field, $\mathrm{t}=27^{\circ}, \mathrm{pH}=5.8,11$ Aug. 1986, A. Shatrovskiy leg. (AR); 1 Õ, Maritime Province, Spasskiy District, Yevseyevka, in stream, 17 Jul. 1976, E. Berlov leg., 'Stenus sp.', 'Stenus vinnulus Cas., det. V. Puthz 2006' (ASh).

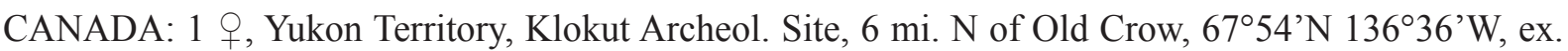
shallow margin of small lake, 19 Jul. 1977, R.E. Morlan, J.V. Matthews, R.E. Roughley leg. (Yukon Refugium Project) (UASM); 1 , ' N.W.T. -21 m.e. Tuktoyaktuk. 17-21.vii.[19]71. D.M. Wood', 'Stenus illotulus Puthz det. V. Puthz 1978', 'ob abw. vinnulus?', 'Eigentum CNC!' <?> (CNC).

\section{Remarks}

Originally described from the USA. When revising Casey's heterogeneous type series, Puthz (1972a) designated the specimen from Cambridge, Massachusetts as the lectotype and placed S. confusoides Renkonen, 1935, that had been known until then from Fennoscandia (Renkonen 1936; Strand 1954; Palm 1961; Puthz 1965, 1970) and E Siberia (Puthz 1967a: Chita Area: 'Dorf Udotschnoje am Ingodazufluss'), in the synonymy of $S$. vinnulus. The specimens from Isle Royale, Lake Superior and Marquette, Michigan, were also attributed to the latter species, but the paralectotypes from White Fish Point were identified as S. brivioi Puthz, which was described in the same year (Puthz 1972c). The same author cited E Siberian 'Poppius-Funde von Ytyk-haja, Ust Aldan und Olekminsk' for S. vinnulus in the same year (Puthz 1972d). Campbell \& Davies (1991), without providing material, reported the species for Alaska and most provinces of Canada, excluding British Columbia, Saskatchewan, Prince Edward 

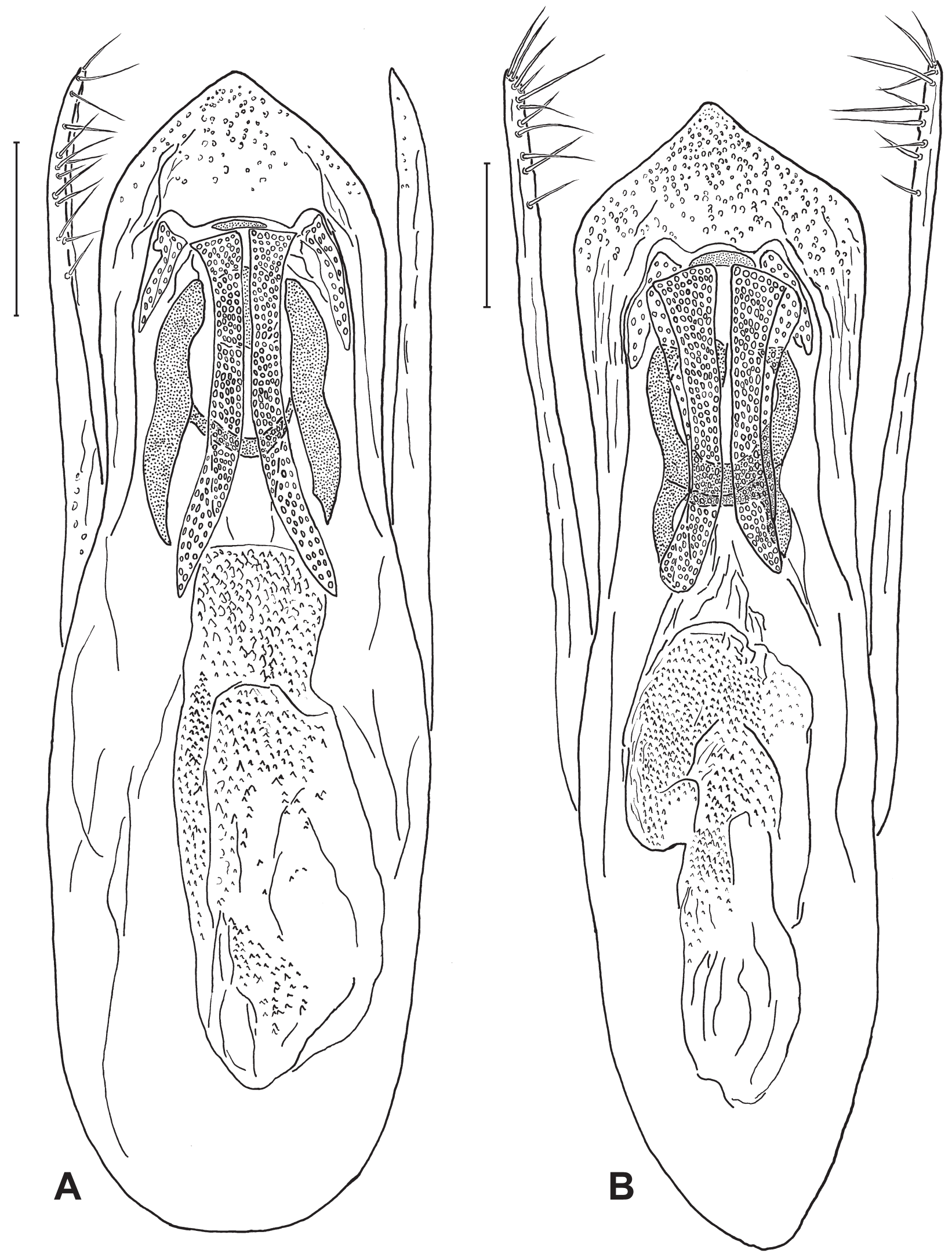

Fig. 6. Aedeagus, ventral view. A. Stenus (Nestus) delitor sp. nov. (holotype). B. S. (N.) sphaerops Casey, 1884 (U.S.A.: New York: Albany Co.). - Scales $=0.1 \mathrm{~mm}$. 
Island, and Newfoundland (the first records for Alaska and the Yukon Territory had been provided by Fall 1926). Ryabukhin (1999) mentioned a single specimen of this species from the Magadan Area, Russia, based on my identifications from the IBPM collection (see Material examined above).

\section{Stenus (Nestus) sphaerops Casey, 1884}

(Figs. 6B, 7I-J)

Stenus sphaerops Casey, 1884: 68.

Stenus (s. str.) sphaerops - Campbell \& Davies 1991: 112.

\section{Material examined}

USA: 1 Oै, 'U.S.A.: New York. S. Westerlo: Bear Swamp. 12VIII:1974. Leg: W. Suter. Albany Co.', 'FM(HD)\#74-195. Berlese: Sphagnum. WS\#74-61D.' (FMNH).

\section{Differential diagnosis}

Based on many external characters, this species might be placed separately within the canaliculatus group. Nevertheless, from the shape and structure of the aedeagus, one can suppose it to be most closely related to the latipennis-illusor-delitor complex. S. sphaerops differs from all the known species of this complex by the broader head with eyes much more convex, by the greater and more bulging pronotum, by the shorter elytra, by the much coarser forebody puncturation, by the less developed ground sculpture, and by the shape of aedeagus.

\section{Redescription}

LENGTH. 4.0-4.1 $\mathrm{mm}$.

Coloration. The single specimen on hand is somewhat immature; therefore, the genuine coloration is probably darker than described here. Body pitchy black, moderately shining, with fairly short silvery pubescence. Legs dark brown with middle parts of femora somewhat lighter, reddish; antennae reddishbrown with $1^{\text {st }}$ segment pitchy black and $2^{\text {nd }}$ segment dark brown; palpi brown, with segment 1 and base of segment 2 yellow.

HEAD. Evidently broader than elytra between humeri (69:62), nearly as broad as elytra in posterior quarter (69:70). Front with a pair of fairly broad and deep longitudinal impressions and a broad keelshaped median elevation that is not very prominent but well developed; median elevation 1.8 times as broad as each of lateral pieces. Puncturation coarse and dense, irregular, partly rugose along internal margins of eyes, with small smooth spot in middle of median longitudinal ridge; diameter of coarsest punctures evidently larger than basal cross of antennal segment 3 . Frontal slope before antennal fossae fairly gentle. Antennae long, reaching basal $1 / 4$ of pronotum. Length proportions of antennal segments $2-11=8: 11.5: 10: 8: 7: 7: 5: 6: 6: 7.5$; segments of club distinctly elongate $(6: 3.5,6: 4.5,7.5: 4)$.

Pronotum. Distinctively large, bulging, uneven, a bit longer than broad (55:52), broadest near middle of length, narrowed convexly anteriorly and concavely posteriorly. Median longitudinal groove deep over almost entire length of disk, vanishing just behind slightly elevated anterior margin. Laterobasal depressions shallow though laterobasal prominences developed. Puncturation coarse, in part nonrugosely obliquely confluent, distinctly greater in middle of length; punctures much larger than those of head.

ELYTRA. Somewhat broader than long (70:63), nearly as broad between humeri as long (62:63), about $1 / 7$ longer than pronotum (63:55) although a bit shorter than pronotum (53:55) by suture. Humeral angles short but prominent, lateral sides slightly convexly and moderately divergent posteriorly (62:70). 
Humeral and sutural depressions rather feeble, vanished posteriorly. Puncturation deep, distinctively larger in diameter than that of pronotum, more regular, partly nonrugosely confluent but not forming long furrows.

LEGS. Rather long; segment 1 of metatarsi obviously longer than segment $5(15: 12)$ though shorter than segments 2-4 together.

ABDomen. Moderately convex, with well developed paratergites and two pairs of keels at bases of anterior visible tergites. Lateral sides uniformly convergent posteriorly. Tergite 7 with very fine light fringe at posterior margin. Puncturation of tergites fairly dense, on anterior visible tergites distinctly sparser medioposteriorly; average diameter of punctures on anterior visible tergites about equal to that of median elevation of head.

MicRosCULPTURE. Very fine but regular mesh-like ground sculpture evident between punctures on abdominal tergites 6-10; other surfaces smooth or with infrequent, extremely vague netting mainly by margins of punctures.

MALE. Meso- and metatibiae without specific characters; abdominal sternites 6-7 slightly depressed along midline and slightly emarginated medioposteriorly; abdominal sternite 8 with broad but not deep, rounded emargination of posterior margin and with flat depression in posterior half; lateral pieces uniformly rounded; abdominal sternite 9 as in Fig. 7J; aedeagus as in Figs. 6B, 7I.

\section{Remarks}

Originally described for the male holotype only: 'Massachusetts'. Campbell \& Davies (1991), without citing material, recorded it for the Canadian provinces Ontario, Quebec, and Nova Scotia, as well as the Northwest Territories. The original description, though fairly long and detailed, did not contain some necessary data comparable to those of present-day publications. Inasmuch as neither descriptions nor figures have been provided for S. sphaerops since Casey (1884), I thought it useful to give such a description here.

\section{Stenus (Nestus) confusus J. Sahlberg, 1876}

(Fig. 2E)

Stenus confusus J. Sahlberg, 1876: 58.

Stenus confusus - Poppius 1899: 39. - J. Sahlberg 1900: 29. — Munster 1921: 119. — L. Benick 1924:

254. — Haberman 1983: 101, 108. — Silfverberg 1988: 20. — Puthz 1998: 149. — Gollkowsky 2001: 194. - Shavrin \& Puthz 2007: 118.

Stenus (Nestus) confusus - Jakobson 1909: 481. — Poppius 1909a: 17. — Renkonen 1935: 29. Hansen et al. 1939: 32. — Palm 1961: 90, 98. — Ryvkin 1987: 159. — Semenov 2004: 12.

Stenus (s. str. + Nestus) confusus - Puthz 1973c: 50.

$<$ ?>Stenus aemulus - Thomson 1857: 224.

Stenus (Nestus) latipennis - Hansen et al. 1939: 32 (pars).

Stenus (Nestus) protensicollis Krása, 1941: 166.

\section{Material examined}

RUSSIA: 1 $\partial$, 'Carelia bor.[ealis] 4122 [=Stenus confusus Sahlb.] - 1.', '4122. confusus Sahlb.' (ZIN); 2 우, 'Petrograd, Smolenskoye cemetery. 11.iv.1920. A.A. Stackelberg leg.', '68'<round>, 'confusus 
Sahlb. L. Benick det.' (ZIN); 1 + , 'Yaroslavl'. 08.vi. N. Filippov.', 'Stenus confusus Sahlb. det. V. Puthz, 1976' (ZMMU); 3 đo ${ }^{\lambda}, 1$ ㅇ, Kalinin [=Tver'] Area, 18 km SW of Kalinin, near Putilovo, Volga River bank, 22 Jul. 1982, I.A. Ushakov leg. (AR); 1 \&, '[Moscow,] on snow in Petrovsko-Razoumovskiy Park, 28.x.1910, V. Boldyrev leg.', 'Stenus nitens Steph.' (ZIN); 1 ㅇ, South of Yamal Peninsula, 110th $\mathrm{km}$ by railway $\mathrm{N}$ off Obskaya station, puddle, 6 Aug. 2001, P. Petrov leg. (AR); 1 \&, Krasnoyarsk Territory, Achinsk, 10 Jul. 1973, V. Zolotikhin leg. (AR); 1 +, '5 verst off Yakutsk, Sergelyakh suburban settlement. 24.viii.1926. L. Bianki', 'Yakutian research expedition of Acad. of Sci.', '47'<round>, 'confusus Sahlb. L. Benick det.' <?> (ZIN); 2우, 'Yakutsk. 22.vi.1925. Bianki', 'Yakutian research expedition of Acad. of Sci.', '63'<rectangle>, 'confusus Sahlb. L. Benick det.' < ?> (ZIN).

\section{Remarks}

Terra typica: Russian Karelia and Finland ('jag har funnit den vid Vigsjön [= 'Lac. Wig'(Silfverberg, 1988)] $\left(63^{\circ} 50^{\prime}\right)$ och Svir i Ryska Karelen, i Kihtelysvaara, Eno och Nurmis socknar i norra Karelen äfvensom vid Jyväskylä och Helsingfors'). In the original description, Sahlberg supposed that Thomson's (1857) record of S. aemulus Erichson, 1839 (see $S$. (N.) nitens above) for Sweden should be referred to S. confusus, but this seems to be doubtful since the species description is quite adequate to $S$. nitens Stephens, 1833, for which aemulus is in fact a synonym. Poppius (1909a) reported the species from the middle reaches of the Lena River: 'An der mittleren Lena an lehmigen Ufern, ein Exemplar bei Jakutsk, 1.VII!, ein anderes auf einer Insel nördlich von Önkyr-yrjä, 8.VII!.' (the East Siberian latipennis specimens, identified by Poppius as confusus and found by Puthz (1972c) in ZMH, were collected at other localities and cannot
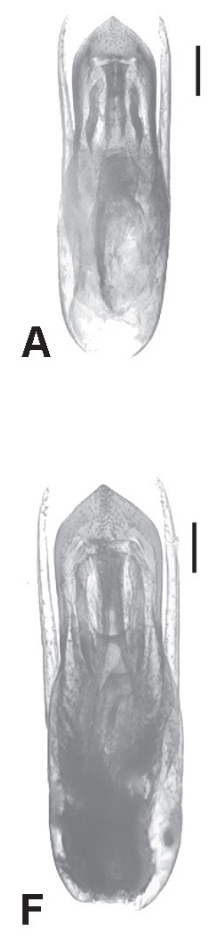
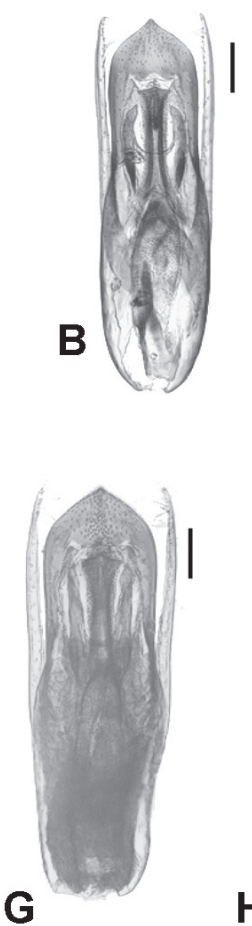

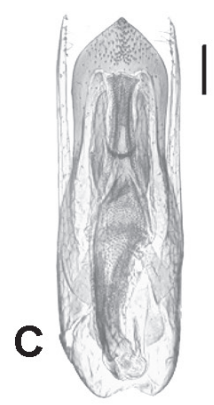

D
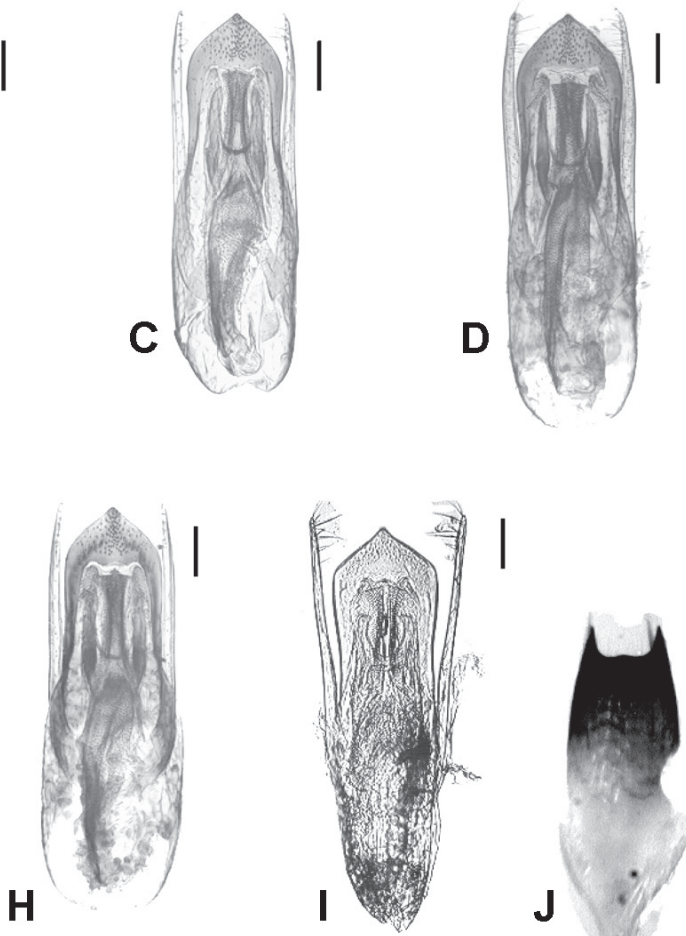
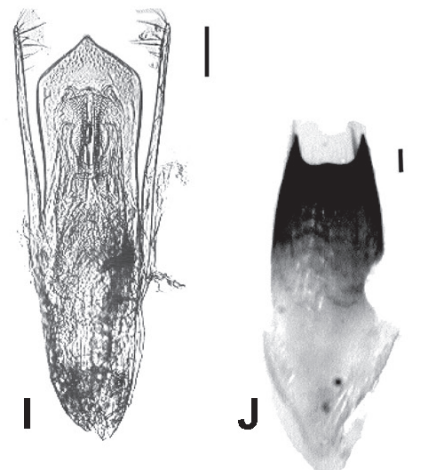
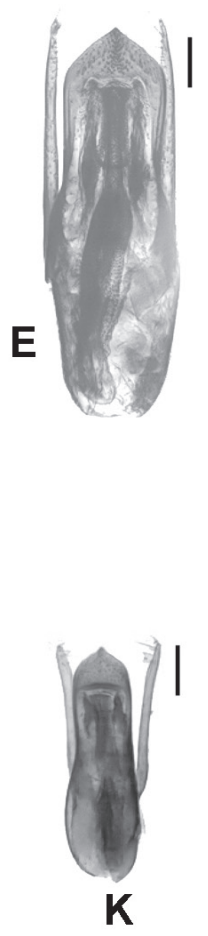

Fig. 7. ô copulative structures. A-I, K: Aedeagus, ventral view. J: Abdominal sternite 9. A. Stenus (Nestus) delitor sp. nov. (holotype). B. S. (N.) illusor Ryvkin, 1987 (Russia: Amur Area: Angelokit River). C-H: S. (N.) latipennis J. Sahlberg, 1880. C. (Russia: SW Taimyr: Ladannakh Lake). D. (Russia: Taimyr: Kotuy River). E. (Russia: Putorana: Ayan Lake). F-G. (Russia: Evenkia: Kruten'kiy Stream). H. (Russia: Magadan Area: 'Aborigen'). I-J. S. (N.) sphaerops Casey, 1884 (U.S.A.: New York: Albany Co.). K. S. (N.) melanopus Marsham, 1802 (Ukraine: Odessa Area: Krinichnoye). - Scales $=0.1 \mathrm{~mm}$. 
be correlated to the material cited in Poppius 1909a). Jakobson (1909) summarised the range as follows: 'Sweden <the record is most likely based on Thomson's misidentification of confusus as aemulus: see above>; Lapponia, Finland, Olonets Government'. Munster (1921) provided some distributional data for northernmost Norway: 'Kaafjord i Alten, Sirma i Tanen samt Neiden, Langfjordbunden, Vaggatim og Graense-Jakobselv i Syd-Varanger !'; L. Benick (1924), a bit later, cited the only female from SE Siberia ('Tschita'); Palm (1961) confirmed N Swedish records of this species (Norrbotten, Jämtland); Haberman (1983) provided three mapped localities from Estonia; a pair of specimens ( $1 \hat{\partial}, 1$ q $)$ have been published recently from the Moscow Area (Semenov, 2004). The terra typica of S. protensicollis Krása, 1941, which was placed in synonymy with confusus (Puthz, 1973c, 1998): 'Simbirsk, Rossia m.[ed]'.

The new material above confirms that $S$. confusus is rather widespread in the Northern Palaearctic, but the species has proved to be rare and sporadic in its distribution. It is essential that all the Siberian records known to me are represented by females only. I expect the range to have a relict pattern.

What is Stenus melanopus (Marsham, 1802)?

\section{Preliminary remarks}

Stenus melanopus has been included in the present paper for the following reasons. Firstly, some authors consider the species to be closely related to the canaliculatus group (e.g. Betz 1.c.); I believe such an interpretation to be not perfectly correct. Secondly, owing to its external resemblance to several other species, including those of the canaliculatus group, $S$. melanopus has been recorded repeatedly for territories where it is in fact not represented. In addition, inasmuch as my study of the material from Stephens' collection (BM) revealed heterogeneity in the series that probably contains the type of S. melanopus, a lectotype designation appears to be called for in order to provide stability in name usage. Lastly, a preliminary analysis of the literature on the species under consideration forces me to call into question some synonyms which have been established earlier and, at least, to raise a question regarding the thorough revision of these synonyms.

A new monotypic species group, the melanopus group, is defined and diagnosed below; its differences from the canaliculatus group, as well as from other groups of the subgenus Nestus, are provided; a lectotype of S. melanopus is designated; a brief analysis of the main published records makes it possible to define the species distribution more exactly and to evaluate the reliability of the synonymy provided.

\section{Definition of the melanopus group}

\section{Diagnosis}

Body size moderate; body length of only known species: 2.8 to $3.7 \mathrm{~mm}$.

Head rather small in comparison to elytra and pronotum. Upper surface between eyes bisulcate, with longitudinal elevations and impressions well developed. Labrum with anterior margin straight, neither sinuate nor notched. Internal tooth of each mandible placed much more distally than middle, turned evidently dorsally of the main plane. Maxillar palpi with basal segment yellow and not shortened. Prementum of normal length, not reduced. Antennae fairly short, with penultimate club segments globular to slightly transverse, the last segment a bit longer than broad.

Pronotum with sharp longitudinal median furrow, rather broad and deep in basal half, vague or vanishing before the middle. 
Elytra moderately long, slightly trapezoid with humeri developed.

Legs moderately long; segment 4 of tarsi without emargination; segment 1 of metatarsi a bit shorter than segment 5 , distinctly shorter than segments 2 to 4 together.

Abdomen moderately convex, with paratergites evident on abdominal segments 3 to 7 ; four anterior visible tergites with four short but evident longitudinal keels at basal part each; posterior margin of tergite 7 with well developed, fine membranous fringe.

Puncturation of pronotum and elytra moderate, non-rugose, with interstices fairly flat.

Ground sculpture entirely absent, upper surface varnish shining.

Pubescence moderate, contiguous.

MALE. Legs without specific features; posterior margin of abdominal sternites 6-7 about straight; abdominal sternite 8 with broad and very shallow, rounded emargination of posterior margin, without evident depression; abdominal sternite 9 with posterolateral teeth very short, not incurved inwards. Aedeagus relatively small; median lobe archetypically lanceolate, with apical sclerotized part angularly rounded; endophallus with paired medial bands and unpaired bar distinct in middle, with very small but visible lateral parts, and with $\mathrm{H}$-shaped expulsion clasp producing fairly broad lateral portions; basal tube evidently sclerotized. Parameres cylindrical, without apical broadening; apico-internal setiferous surface poorly differentiated, setae rather uniform.

Female. Posterior margin of abdominal sternite 8 obtuse-angularly rounded; each valvifer with very short posterolateral tooth, backwards directed. Spermatheca sclerotized.

In the structure of the mandibles, the abdominal tergites, the male genitalia and abdominal sternite 9 of both males and females (valvifera), the melanopus group is most closely related to the atratulusfuscipes-cautus-crassus complex. It differs from all the species of this complex by the presence of a long and sharp, longitudinal median furrow on the pronotum, as well as by the posterolateral teeth of abdominal sternite 9 of both males and females, which are very short and not incurved inwards.

\section{Taxa included}

S. (N.) melanopus (Marsham, 1802).

\section{Bionomics}

See below.

\section{General distribution}

See below.

Stenus (Nestus) melanopus (Marsham, 1802)

(Fig. 7K)

Staphylinus melanopus Marsham, 1802: 528.

Stenus melanopus - Stephens 1829: 290. — Stephens 1833: 299. — Stephens 1839: 411. — Hardy 1851: 45. — Waterhouse \& Janson 1855: 138, 151. — Rye 1864: 41. — Fauvel 1869: 492 (pars). - Rye 1870: 84. — Fauvel 1873: 256. — J. Sahlberg 1876: 59 (pars; original record: false). — Fauvel 1878: 
102 (pars). — Fauvel 1886: 29. — Fowler 1888: 336 (pars). — J. Sahlberg 1900: 29 (false). — Fauvel 1902: 75 (pars). - Sainte-Claire Deville 1906: 82. — L. Benick 1921a: 144. — Wüsthoff 1934: Taf. II. — Normand 1935: 364. — L. Benick 1947: 90. — Focarile 1964: 63. — Puthz 1966: 146. — Puthz 1970b: 174, 178. — Puthz 1972e: 49. — Puthz 1973a: 26. — Anderson 1984: 247. — Lucht 1987: 96. — Puthz \& Zanetti 1995: 18. — Herman 2001: 2278. — Monsevičius \& Pankevičius 2001: 41. — Puthz 2008: 150.

Stenus melanopus<?!> - J. Sahlberg 1880: 78. — Heyden 1881: 78. — Poppius 1899: 39. — Iljin 1926: 225.

Stenus (Nestus) melanopus - Ganglbauer 1895: 576 (pars). — Jakobson 1909: 481 (pars). — Reitter 1909: 158 (pars). — Johansen 1914: 505. — L. Benick 1929: 44 (pars). — Tottenham 1954: 62. — Smetana 1959: 202. — Palm 1961: 88. — Szujecki 1961: 33. — Horion 1963: 339. — Scheerpeltz 1963: 417. — Lohse 1964: 116. — Puthz 1965: 27. — Puthz 1967c: 8. — Puthz 1967d: 305. — Puthz 1971a: 83, 97. — Puthz 1972a: 259. — Tichomirova 1973: 173. — Bordoni 1974: 13. — Dauphin 1993: 187 (pars). — Outerelo et al. 1995: 80. — Bordoni 2004: 119.

Stenus (s. str.) melanopus - Bernhauer \& Schubert 1911: 162 (pars). — Scheerpeltz 1933: 1160. Campbell \& Davies 1991: 111.

$<$ ?>Stenus (Nestus) cribrellus Rey, 1884: 261.

$<$ ? $>$ Stenus nitidus Lacordaire, 1835: 450.

$<$ ? $>$ Stenus nitidus - Erichson 1840: 703. — Kiesenwetter 1845: 224. — Lucas 1846: 122. — Fairmaire \& Laboulbène 1856: 576. — Kraatz 1857: 756. — Thomson 1857: 224. — Kraatz 1858: 123. — Thomson 1860: 225. — Seidlitz 1872-1875(1874): 255. — Seidlitz 1887-1891(1889): 365.

$<$ ?>Stenus (Nestus) nitidus - Rey 1884: 256.

$<$ ?>Stenus sulcicollis Stephens, 1833: 295 (nomen dubium).

Stenus tythus Schaufuss, 1882: 621.

\section{Material examined}

UK: Lectotype (designated herein): $q,<$ small square of red paper $>$, '53' $<$ hand-written with black Indian ink on small white oval>, 'British Isles. J. Stephens Coll. BM 1853-46'<printed rectangular label>, 'Standing in Stephens Coll. as Stenus melanopus Marsham' <printed rectangular label>, 'LECTOTYPUS' < my standard printed red label>, 'LECTOTYPUS | Stenus | melanopus Marsham | A.B. Ryvkin des. $2003^{\prime}<$ my standard printed determinative label $>$ (BM).

FRANCE: 1 + , '806', 'Stenus | melanopus | Marsh. | Gallia' [ex coll. Semenov-Tian-Shanskiy] (ZIN); 1 ․, 'Corsica. 4121 [=Stenus melanopus Marsh.] - 2.', ‘4121. melanopus Marsh.’ (ZIN).

UKRAINE: 1 ${ }^{\lambda}, 3$ $\rightarrow+$, Odessa Area, Bolgradskiy District, near Krinichnoye, fresh-water lake shore, in reed drift, 5 May 2003, A.V. Gontarenko leg. (AR).

ALGERIA: 1 ○े, '186', '? Algeria | ? Deyrolle.' (ZIN).

\section{Measurements of the lectotype}

Entire body length: $3.1 \mathrm{~mm}$; head width: 48 ; pronotum length: 45; pronotum width: 45 ; elytral length: 59; elytral length by the suture: 50; elytral width between humeri: 53; maximum elytral width (in posterior $1 / 4): 64$. 


\section{Remarks}

The lectotype of S. melanopus is designated from among the four specimens under this name in Stephens' collection and originating likely, at least in part, from Marsham's collection. Only this female belongs to melanopus in the commonly accepted interpretation. Only this specimen has been marked with a small patch of red paper and only for this specimen is the number ' 53 ' indicated, which corresponds to the number of the species in Stephens' 'Illustrations of British Entomology'; it is believed to have been placed by Waterhouse on a small white oval label, without additional daggers. I have transferred it to a new rectangular board; the old, smaller board remains on the same pin.

Similar problems that have arisen during the revision of the collections of Marsham, Kirby, and Stephens have been discussed many times by many authors (e.g. Tottenham 1937; Hammond 1972). An excellent historical reference was kindly compiled by Mrs. Sharon Shute (Department of Entomology, BM), in addition to the material received by me on loan in 2002. Below, I give a slightly abbreviated version of the text of this reference with the permission of the author and management of the Department [e-mail of February 20 2004]:

\section{"Stenus melanopus Marsham}

This species was described by Marsham 1802. Stephens redescribes this species, in Illustrations of British Entomology [...]. Stephens acquired specimens from Marsham's collection when it was sold after his death and normally indicates in his text if he had a Marsham specimen. The species name in the Illustrations is attributed to Marsham, however, he does not mention that he had Marsham's specimen. The Staphylinidae in the Stephens collection normally bear small, white, round labels if they come from the Marsham collection, (see Hammond 1972[...]). It is thought these were put on by Stephens, although they do not occur on the specimens of the species of many other families in his collection although we know from Stephens sale list of the Marsham collection that he did indeed purchase specimens of many of these species. The larger oval white labels with black ink numbers are (? Waterhouse) attached to all four specimens of this species in the Stephens collection. The numbers on these labels refers to the species number in The Illustrations Vol. v. there is no indication what the asterisks mean. None of these specimens have a Marsham round, white label, whereas other species within this genus do. This is a mystery because Waterhouse in his paper with Janson, on some of the British Stenus species, [...], says that the 'type' is in the Stephens collection. This may have been an assumption on his part and he may not have known what the round, white labels meant. The fact that Stephens does not mention having Marsham's specimen at the time of the redescription I think can be taken to assume he did not have it in his collection. Although the series label is not in his hand and could be Marsham's ???. [...] It may be advisable to select a neotype from the Stephens collection as without a label on the specimen it will not be possible to identify which if any of the four specimens came from the Marsham collection. Please note that as the specimens in the Stephens and Kirby collections do not have any locality data it is not possible to tell if the specimens are those he had in front of him at the time of his descriptions or later additions. It is known that he exchanged material and often disposed of specimens if he collected 'better' ones which is why in a number of cases it has been found that the specimens standing as a particular species in Stephens collections do not fit his description. Care must be taken when using these specimens to 'fix' names, to take account of common usage of names and the common interpretations of such species before changing names as a result of examining Stephens specimens, which should in every case be carefully checked against Stephen's description. [...]" [e-mail of May 16 2002]

Unfortunately, Marsham's original description cannot help sufficiently with identification of the original type specimen: 'St. niger obscurus scaber, pedibus concoloribus. Long. corp. 11/2 lin. Habitat -----. Descr. Simillimus St. immuni, at pedes omnino nigri.' Every third Stenus species appears to conform to the diagnosis cited. Even the reference to $S$. immunis, described by Marsham in the same monograph, can add little clarity to solving this problem. The latter name, having been synonymized with no evident 
proof with either S. (Nestus) circularis Gravenhorst, 1802 or S. (Hemistenus) pallipes Gravenhorst, 1806 , at different times, requires a separate revision in itself.

The redescription by Stephens (1833), on the contrary, is much more detailed and corresponds well, in main features, to the melanopus in commonly accepted interpretation. The only inconsistency may be found in the coloration of the maxillar palpi: 'antennae and palpi black, immaculate' whereas the basal segment of the palpi is in fact yellow. The following information provides an explanation for this inconsistency: 'It is said of Stephens that he declined to use a microscope regularly believing that what could not be seen with the naked eye was not worth studying' (Darby 2010). I can confirm that the colour of the basal segment of the palpi may be poorly visible in a total specimen even at a high magnification. The redescription given by Waterhouse \& Janson (1855) 'according to the type specimen in Stephens' Coll.' relates undoubtedly to $S$. melanopus in commonly accepted interpretation and conforms to the unique female mentioned above. It is necessary to remember that the paper by Waterhouse \& Janson (1855) was published just about three years after the death of Stephens, by authors who had been close to him and had known his collection for many years. Thus, both the redescriptions obviously indicate that Stephens had at least a specimen of the 'true' melanopus in front of him at the time of his redescription.

I suppose 'the label problem' to be confused in this case not so far as the author of the aforementioned historical reference finds. Waterhouse \& Janson (1855) reported that 'the specimens which Mr. Stephens used to call his 'Type specimens' are almost always marked by some kind of ticket attached to the pin holding the insect. The Marshamian specimens are thus marked by a round yellow ticket; and when the species is described in the 'Entomologia Britannica', a number will be found on the under side of the ticket, corresponding to the number of the species in that work. Other type specimens are either marked by a round white ticket without a number, or by a small square ticket with a number. Mr. Stephens' own species are not marked'. I should like to stress the words 'almost always' in the text cited. In my opinion, they may prove that not all the 'type specimens' in the collection were actually marked in the way they described. About the small round labels Hammond (1972) mentions: 'All internal evidence from the collection suggests that the colour of these labels is of no great significance but that they all indicate specimens originating from Marsham's collection'; however, he did not present valid arguments against the possibility that specimens without such round tickets or having labels of other shapes may also have belonged to the named collection. The same author further stated: 'The significance of certain other labels attached to various specimens, including white rectangles bearing a number and small red squares of paper, has not been discovered'; however, these 'white rectangles' may have conformed to the 'small square tickets with a number' in the aforecited fragment by Waterhouse \& Janson (1855). Apropos, the latter authors did not indicate a colour of the 'tickets', and the small red squares of paper may be analogs of the white rectangles: as we can see in the text, Waterhouse \& Janson did not regard the numbers as indispensable attributes of the type labels.

Stephens never stated that all the material from Marsham's collection would be specified separately in his 'Illustrations'. Indeed, he mentioned such material directly in the Stenus section in the following three cases:

1. under S. marshami Stephens, 1833 (=S. (Tesnus) brunnipes Stephens, 1833; =S. immunis Marsham, 1802, pars); it was, at least in part, based on material in the series of immunis from Marsham's collection (see the 'Systematic Catalogue': Stephens 1929: 289);

2. under S. immunis Marsham, 1802 (the name requires a revision, see above); since the series proved to be heterogeneous, it was reasonable to show that a part of the original series remained under the name of immunis; besides, since Marsham's descriptions did not contain precise references to localities, Stephens gave additional data (London \& Norfolk), having mentioned that his own specimens came 'from the Marshamian collection'; 
3. under S. laevis Stephens, 1833 (=S. (Metatesnus) pubescens Stephens, 1833), which he attributed to Marsham and therefore, to explain the posthumous attribution, found it appropriate to mention that his specimen had been 'obtained from the Marshamian cabinet'.

However, I can find no reasons for particularly citing material from Marsham's cabinet under the name melanopus: the species was thoroughly described and published by Marsham. It was a well known fact that Stephens had purchased Marsham's collection. In this case, Stephens merely gave the redescription more detailed based on the 'type specimen' and then added: 'Also found, not uncommonly, within the metropolitan district and in Norfolk', with a reference to the habitat data provided by L. W. Dillwyn, Esq. The word 'also' here means, in my opinion, that the above redescription is based on Marsham's original material.

Based on the statement by Waterhouse \& Janson (1855), as well as on all the facts aforesaid, I consider the specimen under discussion to be an original type specimen that can be designated as the lectotype.

The remaining three specimens ( 1 + of Stenus (Tesnus) brunnipes Stephens, 1833; 1 o, 1 q of Stenus (Nestus) boops Ljungh, 1810) have not been marked as paralectotypes inasmuch as their belonging to the original type series seems improbable; all those have been supplied with my common determinative labels. Each of these specimens has also been labelled by Waterhouse: 'The species follow in succession, in the cabinet, in accordance with the descriptions in the "Illustrations," but in one or two instances there have undoubtedly been some accidental transpositions; and to prevent further changes of this nature, all the species, and indeed nearly all the specimens, have now been numbered to correspond with the numbers of the species as given in the "Manual." [=Stephens 1839]. The numbers here alluded to are on small oval tickets attached to the specimens [...]' (Waterhouse \& Janson 1855). Besides the number '53', the oval individual labels bear additional markings: one dagger under $S$. brunnipes; two daggers (one under another) under each of the specimens of $S$. boops. The daggers may have marked extraneous species within each series.

In different times different species have been erroneously synonymized with S. melanopus: S. (N.) capitatus Eppelsheim, 1878 (Fauvel 1878, 1902; Fowler 1888; Ganglbauer 1895; Reitter 1909), $S$. (N.) discretus Rey, 1884 (now a synonym of $S$. (N.) crassus Stephens, 1833) (Ganglbauer 1895; Fauvel 1902; Reitter 1909; Bernhauer \& Schubert 1911; L. Benick 1929; Dauphin 1993), S. (N.) ignotus Eppelsheim, 1890 (Fauvel 1902), S. (N.) piscator Saulcy, 1864 (Fauvel 1902), S. (N.) arctulus Hochhuth, 1849 (now a synonym of $S$. (N.) incanus Erichson, 1839) (Fauvel 1902), S. (N.) morulus Baudi, 1870 (now a synonym of S. (N.) piscator Saulcy, 1864) (Fauvel 1902) and S. (N.) sulcifrons Eppelsheim, 1878 (now a synonym of $S$. (N.) piscator Saulcy, 1864) (Fauvel 1902). Among the names having been regarded as synonyms of $S$. melanopus till now, only $S$. tythus Schaufuss, 1882 does not raise doubts: its female type was studied and identified as melanopus by Puthz (1967c). None of the names S. cribrellus Rey, 1884, S. nitidus Lacordaire, 1835 or S. sulcicollis Stephens, 1833 have been revised based on the type material.

The last of these seems to be a nomen dubium. Waterhouse \& Janson (1855: 148-149) indicated: 'The detailed description in this work is possibly from a small specimen of St. [(Hemistenus)] subaeneus, Er. The insect in Mr. Stephens' cabinet which stands for sulcicollis evidently is misplaced by a black-legged species, i.e. St. melanopus, of Marsham'. The result of this mess was marked well by Rye (1870): 'This [sulcicollis] and the preceding [assimilis] are given as valid species in Gemm.[inger] \& v. Harold's Cat.[alogue], ii, though (? because) they are not recognizable or known to British Entomologists. The former is represented by brunnipes, and the latter by melanopus, in Stephens' collection. See Wat. \& Janson ([...] 1855), from whose account of the confusion with regard to these insects it is evidently impossible to do anything but ignore them altogether.' Fauvel (1869), without any explanation, put the 
name sulcicollis in the synonymy of melanopus. The assumption by Puthz (2008) that Fauvel had seen the 'type' in Stephens' collection before synonymizing sulcicollis ('FAUVEL hat das 1869 wohl ebenso gesehen, sonst hätte er seine Synonymie nicht ausgesprochen') is contrary to fact: unlike many other names, which are supplied with remarks 'ex typ.' in the paper cited, the pair 'S. sulcicollis Steph. = S. melanopus Marsh.' has no such remark. In addition, the reference by Puthz (2008) to the 'indisputable authority' of Fauvel, who actually had the name of 'master of false synonymy' (Lohse 1985; see, for example, the list of erroneous synonyms above) seems not to be the best way to confirm 'ausgesprochene Synonymie.' By the way, in the later work (1873), Fauvel himself discussed the problem much more deliberately, with a reference to Rye (1870): 'Le sulcicollis Steph. (Ill. Brit., V, 295), représenté dans la collection Stephens par le melanopus, est une espèce méconnaissable à rayer des catalogues $(\mathrm{V}$, Rye, Ent. Annual, 1870, 84)'. Bernhauer \& Schubert (1911), based on the original description, placed S. sulcicollis within the subgenus Hemistenus Motschulsky, 1860 (s.l.) as a valid species. Thus, I find the synonymy of $S$. sulcicollis with $S$. melanopus to be ill-founded.

The original descriptions of both $S$. cribrellus and S. nitidus correspond rather to species of the atratulus complex than to $S$. melanopus: 'un exemplaire [...] à prothorax paraissant un peu plus court, subtransverse, avec une fossette poncti-forme, obsolète, seulement visible suivant un certain jour et située près de la base [...]' (Rey 1884); 'Prothorax un peu plus long que large, [...] avec une fossette oblongue peu marquée sur le disque [...]' (Lacordaire 1835). It is obvious that the small 'fossette ponctiforme, obsolète' as well as 'fossette oblongue peu marquée' cannot conform with the long and deep furrow on the pronotum in $S$. melanopus. Therefore, most subsequent records of $S$. nitidus may have been the result of misidentification of $S$. melanopus. Thus, S. tythus may be regarded as the only reliably established synonym of $S$. melanopus.

This species, widely distributed over the Mediterranean, W \& C Europe, and also introduced into the Nearctic (see Puthz 1966; Campbell \& Davies 1991), is rather variable and similar to many other representatives of different groups in many characters; therefore the range: 'Mittel-Südeuropa, südliches Nordeuropa, Nordafrika, Kanaren, Kleinasien, Westsibirien, östliches Nordamerika'(Puthz 1971a, 1972), seems to be greatly exaggerated. J. Sahlberg (1876) first recorded S. melanopus for S Finland ('Sällsynt; funnen några gånger invid Åbo af O.Reuter, och förf.[attare]'). The same data ('Regio Aboënsis') was cited by him in the subsequent Catalogue (J. Sahlberg 1900). Although L. Benick (1921a) ascertained that this record had resulted from misidentification ('Ein von Sahlberg [...] genanntes Stück (Reuter, Åbo) in der Sammlung des Mus. Hels. ('Reuter, Pargas') ist atratulus Er.'), many subsequent authors continued to cite Finland in the range description of melanopus (Palm 1961; Horion 1963; etc). Benick (1.c.) soundly questioned also the record by Poppius (1899: 'statsradet $A$. Günther [...] i trakten kring Petrosawodsk') for N European Russia ('Die Angabe von Poppius, nach der die Art in Russ. Karelien [...] vorkommen soll, ist nachzuprüfen'). In his well-known work on Siberian Coleoptera, J. Sahlberg (1880) found S. melanopus to be distributed over the Ob' basin ('In territorio silvoso prope oppidum Tobolsk 3/6 unicum specimen invenit Bergroth'). This misleading record was repeated by Heyden (1881) and became a source of erroneous citation of this species for W Siberia by many authors up to present time (Jakobson 1909; L. Benick 1929; Scheerpeltz 1933; Smetana 1959; Szujecki 1961; Horion 1963; Puthz 1971a, 1972; Tichomirova 1973; Dauphin 1993). Neither Puthz (pers. comm.) nor I have seen melanopus specimens from territories eastwards of Denmark and southernmost Sweden. One can confidently suppose the N Russian and Siberian records to relate to another species; most likely, it may be a species of the canaliculatus group, either S. labilis or S. confusus.

The record for Yekaterinoslav [=Dnepropetrovsk] of the Ukraine (Iljin 1926) may also be a result of misidentification. The only material I have seen from the territory of the former USSR is given in the Material section (see above: Odessa Area). The recent record of this species for Lithuania (Monsevičius \& Pankevičius 2001: 1 specimen of undisclosed sex) requires confirmation. 
The bionomics of this species seem to be rather diverse. Along with different open alluvia: seashore, riverside, and lakeside (Hardy 1851; Rye 1864; Fauvel 1873; Fowler 1888; Johansen 1914; L. Benick 1929, 1947; Palm 1961; Horion 1963; Anderson 1984; Dauphin 1993), many other types of habitats have been reported: hay-stack rubbish (Rye 1864), manure heaps (Tottenham, 1954), dry manure (Dauphin 1993), compost heaps (Anderson 1984), detritus (Smetana 1959; Dauphin 1993), humus (Bordoni 1974) and bogs, including peat and Sphagnum (Horion 1963).

The suppositions of halophily for S. melanopus (L. Benick 1947; Puthz 1965, 1971, 1972) seem to be ill-founded, inasmuch as inhabiting seashore alluvia and lakesides of brackish basins is not a sufficient ground to consider a species to be halophilous. The idea was argued and rejected by Horion (1.c.: 'Von einer allgemeinen Halophilie dieser Art kann keine Rede sein'). I believe that the fact that $S$. melanopus inhabits Sphagnum bogs is quite enough for the assumption of halophily to be denied.

As to the records of the occurrence of this species in manure and compost heaps, I should like to note that those habitats are common shelters which many Stenus species use during adverse weather or in adverse seasons.

\section{Key to the Palaearctic species of the canaliculatus group}

1. Front with deep longitudinal impressions and high median elevation in between. Seventh abdominal tergite varnish shining, without ground-sculpture between punctures. Puncturation of pronotum and elytra fairly deep and coarse, average diameter of punctures on pronotum larger than basal cross of antennal segment 3. Disk of pronotum with deep longitudinal furrow rather broad and not lineshaped throughout. Internal tooth of each mandible shifted distally, directed mediodorsally of the main plane. Abdominal sternite 9 of both males and females (valvifera) with posterolateral teeth very short. Aedeagus small (see Fig. 7K). 2.8-3.7 mm .

.[melanopus group] melanopus (Marsham, 1802)

- If front with deep longitudinal impressions and $7^{\text {th }}$ abdominal tergite between punctures without regular ground sculpture, puncturation of pronotum very fine and dense with average diameter of punctures distinctly smaller than basal cross of antennal segment 3 . Longitudinal median furrow of pronotum more or less deep but always long, line-shaped. Internal tooth of each mandible placed a little before the middle, directed only slightly dorsally of the main plane. Abdominal sternite 9 of both males and females (valvifera) with posterolateral teeth normally developed (see Fig. 5A-E). Aedeagus large [canaliculatus group] 2

2. Front with distinct lateral impressions and median elevation in between .3

- Front feebly convex or feebly concave, with median elevation and lateral depressions rather vague or entirely absent .....

3. Median elevation of front broad and knob-like. Puncturation of pronotum very fine and dense, average diameter of punctures distinctly smaller than basal cross of antennal segment 3. Disk of abdominal tergite 7 without regular ground sculpture or with reticulation very feeble and vague. Aedeagus as in Fig. 1E. 3.3-4.5 mm labilis Erichson, 1840

- Median elevation of front ridge-shaped, angular in its cross section, flanked by broader and less sharp lateral impressions. Puncturation of pronotum coarser, average diameter of punctures about as large as or larger than basal cross of antennal segment 3 .4

4. Apical abdominal tergites without ground sculpture. For aedeagus, see Puthz 2006 (fig. 4). 2.9-4.0 $\mathrm{mm}$ immigratus Puthz, 2006 
RYVKIN A.B., Stenus species of the canaliculatus group

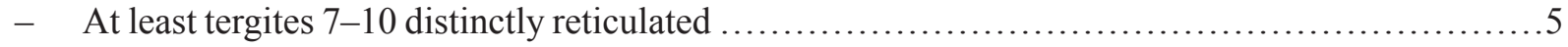

5. Elytral suture about $1 / 4-1 / 5$ longer than pronotum. Aedeagus as in Fig. $7 \mathrm{C}-\mathrm{H}$. $4.0-4.7 \mathrm{~mm}$ latipennis J. Sahlberg, 1880

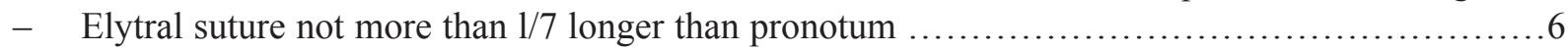

6. Elytral suture a bit shorter than pronotum. Aedeagus as in Figs. 6A, 7A. 4.0-4.6 mm ...delitor sp. nov.

- Elytral suture distinctly longer than pronotum ...........................................

7. Elytra more than $1 / 4$ broader than head. Puncturation less coarse, much more regular; interspaces between punctures along the midline of anterior visible tergites distinctly wider than average diameter of punctures. Aedeagus as in Fig. 2E. 3.4-4.0 mm ..............confusus J. Sahlberg, 1876

- Elytra by $1 / 4-1 / 5$ broader than head. Puncturation much coarser, distinctly irregular; interspaces between punctures along the midline of anterior visible tergites smaller than average diameter of punctures. Aedeagus as in Fig. 7B. 3.9-4.4 mm illusor Ryvkin, 1987

8. Elytra not longer than pronotum, nearly as broad as head. Abdominal tergites with very dense and deep ground-sculpture. Aedeagus as in Fig. 2C, D. 3.5-4.7 mm (the latter value for specimens with the abdomen extended) ...............................illotulus Puthz, 1972

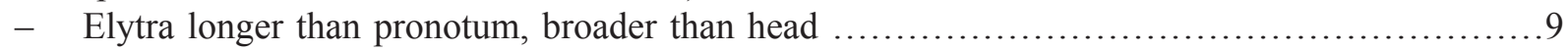

9. Front as a whole feebly concave, with median elevation very vague, nearly flat, situated below internal margin level of eye; the upper surface except abdominal tergite 10 without ground sculpture. For aedeagus, see Puthz, 1987 (Abb. 1). 3.3-3.7 mm .........................shogun Puthz, 1987

- If front as a whole concave, at least abdominal tergites 7-8 with evident ground sculpture ......10

10. Front with median elevation and lateral depressions rather feeble but evident. Median parts of anterior abdominal tergites with puncturation very small, scattered, and shallow. For aedeagus, see Renkonen, 1935 (fig. 1). 3.0-3.8 mm .............vinnulus Casey, 1884

- Front with median elevation entirely absent or, occasionally (in S. nitens Stephens, 1833), represented by narrow, smooth longitudinal strip ....................................... 11

11. Elytra with fairly small and dense puncturation. Anterior margin of labrum with a small median

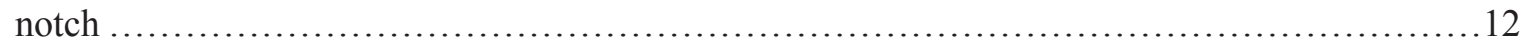

- Elytra with puncturation larger and more or less sparse. Anterior margin of labrum without

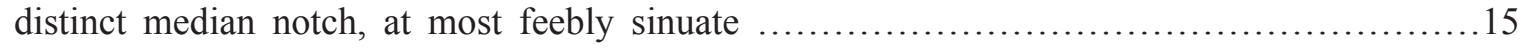

12. Pronotum 1/7-1/8 longer than broad. Aedeagus as in Fig. 3A, B. 3.2-3.9 mm (the latter value for specimens with abdomen extended) ..................................alopex sp. nov.

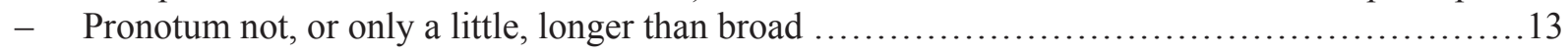

13. Entire body with ground sculpture more or less visible. Aedeagus as in Fig. 3D. $3.0-4.5 \mathrm{~mm}$ (the latter value for the American specimen with abdomen extended, the maximum value for Palaearctic specimens known to me: $4.3 \mathrm{~mm}$ ) canaliculatus Gyllenhal, 1827

- At least median part of abdominal tergites 3-7 without traces of microsculpture .................14

14. Elytra between punctures with ground sculpture throughout. Aedeagus as in Fig. 4A, B. 4.0 $\mathrm{mm}$ (with abdomen extended) ........................................................

- Elytra between punctures with ground sculpture mainly in humeral impressions and near suture. Aedeagus as in Fig. 4C, D. 2.8-3.7 mm (the latter value for specimen with abdomen extended) canalis sp. nov. 
15. Middle of front punctured much sparser than lateral parts, occasionally with a small longitudinal strip or spot broader than the average diameter of punctures. Elytra only a bit (1/14th to $1 / 15$ th) broader than head, being punctured larger and sparser. Aedeagus as in Fig. 1A-D. 3.4-4.0 mm (the latter value for specimens with the abdomen extended) .................nitens Stephens, 1833

- Front with puncturation more regular, without evident smooth strips or spots. Elytra 1/7 to 1/10 broader than head, being smaller and denser punctured. Aedeagus as in Fig. 2A, B. 3.1-4.1 mm (the latter value for specimens with the abdomen extended) raddei Ryvkin, 1987

\section{Ackowledgements}

I wish to thank V.V. Belov, K.Yu. Eskov, S.A. Kurbatov, S.L. Kuz'min, P.N. Petrov, R.A. Rakitov, L.B. Rybalov, V.B. Semenov, S.A. Serbenyuk, A.V. Sokolov, I.D. Sukacheva, K.P. Tomkovich, I.A. Ushakov (Moscow), O.N. Kabakov, B.A. Korotyayev (St. Petersburg), E.A. Khachikov, V.I. Lomakin (Rostov-on-Don), L.N. Dubeshko, V.G. Shilenkov (Irkutsk), E.G. Matis, D.I. Berman, A.S. Ryabukhin (Magadan), A.V. Shavrin (Daugavpils), S.V. Saluk (Minsk), A.V. Gontarenko (Odessa), A.G. Shatrovskiy (Kharkov), S.Ya. Blinshtein (Dortmund), A.Yu. Solodovnikov (Copenhagen), L. Penev (Sofia), the late A.L. Tichomirova, P.K. Yeryomin, V.V. Zherikhin, for the material donated.

I am indebted to the curators and holders of the collections named in the Material and methods section, who gave me an opportunity to study respective material.

I express my profound gratitude to Mrs. Sharon Shute (BM) for the exhaustive historical reference on the series of S. melanopus Marsham, 1802, as well as to Martin Brendell (BM) for useful advice concerning the specimens. I am thankful to Alexey Solodovnikov (Copenhagen), who has supplied me with a copy of Marsham's original description of S. melanopus, and to Oliver Betz for the reprints of his splendid works.

I am indebted also to the administration and staff of the aforenamed nature reserves who have assisted in my own field work in these protected areas.

My best thanks are due to my dear colleagues Arnaldo Bordoni (Florence), Adriano Zanetti (Verona), Alexey Shavrin (Daugavpils) for information concerning material; and also to Volker Puthz (Schlitz) for various useful data including the photo of the aedeagus of S. sphaerops Casey, 1884 from his collection.

And finally, I am perpetually indebted to my wife and colleague Elena Veselova for the help I have received both in our common collecting trips and in our joint office study.

\section{References}

Anderson R. 1984. Staphylinidae (Coleoptera) in Ireland. 3: Steninae. The Irish Naturalist's Journal 21(6): 242-251.

Benick L. 1921a. Über nord-palaearktische Steninen, vorwiegend aus dem Zoologischen Museum in Helsingfors (Col., Staphyl.). Mit 4 Fig. Meddelanden af Societas pro Fauna et Flora Fennica 46(19191920): 135-156.

Benick L. 1921b. Über Stenus-Typen von Mäklin und Motschulsky aus dem Zoologsichen Museum in Helsingfors, nebst Beschreibung einer neuen Art (Col., Staphyl.). Entomologische Blätter 17: 112-116.

Benick L. 1924. Friebs Stenus-Ausbeute in sibirischer Kriegsgefangenschaft. Deutsche Entomologische Zeitschrift: 249-258. 
Benick L. 1925. Bemerkungen zum Catalogus Coleopterorum regionis palaearcticae, Unterfamilie Steninae. Koleopterologische Rundschau 11(3/4): 71-76.

Benick L. 1926. Neue Megalopsidiinen und Steninen vorwiegend aus dem Zoologischen Museum in Hamburg (Col.). Entomologische Mitteilungen 15: 262-279.

Benick L. 1929. Steninae (Staphyl.). Bestimmungs-Tabellen der europäischen Coleopteren 96: 1-103.

Benick L. 1938. Die Steninen Mittelamerikas (Col., Staph.). Mitteilungen der Münchner Entomologischen Gesellschaft 28: 247-281.

Benick L. 1947. Über den Massenwechsel und die Verbreitung norddeutscher Käfer. Forschungen der Geographischen Gesellschaft und des Naturhistorischen Museums in Lübeck (2)41: 84-112.

Bernhauer M. \& Schubert K. 1911. Staphylinidae. II. In: Schenkling S. (ed.) Coleopterorum Catalogus. Pars 29: 87-190. W. Junk, Berlin.

Betz O. 1996. Function and evolution of the adhesion-capture apparatus of Stenus species (Coleoptera, Staphylinidae). Zoomorphology 116: 15-34. http://dx.doi.org/10.1007/BF02526926

Blackwelder R.E. 1943. Monograph of the West Indian beetles of the family Staphylinidae. United States National Museum, Bulletin 182: i-viii + 1-658. http://dx.doi.org/10.5479/si.03629236.182.i

Bordoni A. 1974. Gli Stenus della Toscana (Col., Staphylinidae) (XIII contributo alla conoscenza degli Staphylinidae). Bolletino. Associazione romana di entomologia 29(1/2): 1-28.

Bordoni A. 2004. Atlante degli edeagi degli Stenus della fauna italiana (Coleoptera, Staphylinidae). Bolletino della Società entomologica italiana 136(2): 101-140.

Campbell J.M. \& Davies A. 1991. Family Staphylinidae. Rove beetles. In: Checklist of beetles of Canada and Alaska. Publication 1861/E: 86-124. Agriculture Canada, Ottawa.

Casey Th.L. 1884. Revision of the Stenini of America north of Mexico. Insects of the family Staphylinidae, order Coleoptera. Collins Printing House, Philadelphia. 206 pp. http://dx.doi.org/10.5962/bhl.title.9208

Casey Th.L. 1892. Coleopterological notices. IV. - Annals of the New York Academy of Sciences 6: 359-712. http://dx.doi.org/10.1111/j.1749-6632.1892.tb55408.x

Darby M. 2010. Biographical Dictionary of British Coleopterists. http://coleopterist.org.uk/biogdict/s. htm.

Dauphin P. 1993. Notes sur les Stenus français (Coleoptera Staphylinidae). 2. Le sous-genre Nestus. L'Entomologiste 49(4): 177-192.

Dubeshko L.N. 1984. Zhestkokrylyie v biotsenozakh del'ty reki Selengi. [In Russian]. In: Shilenkov V.G. (ed.) Zhestkokrylyie Sibiri: 46-63. Irkutsk State University, Irkutsk.

Eppelsheim E. 1880. A list of beetles of the family Staphylinidae having been found in Yaroslavl' Gouvernment. [In Russian]. Proceedings of the Society for Exploration of Yaroslavl' Government with respect to natural history 1: 90-95.

Eppelsheim E. 1893. Beitrag zur Staphylinen-Fauna des südwestlichen Baikal-Gebietes. Deutsche Entomologische Zeitschrift 1: 17-67.

Erichson W.F. 1839. Die Käfer der Mark Brandenburg. Erster Band. Zweite Abteilung: 385-740. F.H. Morin, Berlin.

Erichson W.F. 1840. Genera et species staphylinorum insectorum coleopterorum familiae: 401-954. F.H. Morin, Berlin. http://dx.doi.org/10.5962/bhl.title.59644 
Fairmaire L. \& Laboulbène A. 1856. Faune entomologique française ou description des insectes qui se trouvent en France. Coléoptères. Tome premier [liv. 3]: 371-665. Deyrolle, Paris.

Fall H.C. 1926. Additions to the list of Alaskan Coleoptera taken in the summer of 1924. The PanPacific Entomologist 3: 59-63.

Fauvel A. 1865. Énumération des insectes recueillis en Savoie et en Dauphine (1861-1863) et descriptions d'espèces nouvelles. Bulletin de la Société Linnéenne de Normandie 9: 253-321.

Fauvel A. 1869. Remarques synonymiques sur les staphylinides du Catalogus Coleopterorum de MM v. Harold et Gemminger. L'Abeille. Mémoires d'Entomologie 5: 479-494.

Fauvel A. 1873. Faune Gallo-Rhénane ou species des insectes qui habitent la France, la Belgique, la Hollande, le Luxembourg, la prusse Rhénane, la Nassau et la Valais avec tableaux synoptiques et planches gravées. 3, liv. 4: 215-390. Le Blanc-Hardel, Caen.

Fauvel A. 1878. Les Staphylinides de l'Afrique boréale. Bulletin de la Société Linnéenne de Normandie (3)2: 83-162.

Fauvel A. 1886. Les Staphylinides du Nord de l'Afrique. Revue d'Entomologie 5: 9-100.

Fauvel A. 1889. Liste des Coléoptères communs a l'Europe et a l'Amérique du Nord d'après le catalogue de M. J. Hamilton avec remarques et additions. Revue d'Entomologie 8: 92-174.

Fauvel A. 1902. Catalogue des Staphylinides de la Barbarie de la Basse-Égypte et des Iles Açores, Madères, Salvages et Canaries (5édition). Revue d'Entomologie 21: 45-189.

Focarile A. 1964. Ricerche coleotterologiche sul litorale ionico della Puglia, Lucania e Calabria. Campagne 1956-1957-1958. X. - Coleoptera Staphylinidae. Bolletino della Società Entomologica Italiana 94: 49-70.

Fowler W.W. 1888. The Coleoptera of the British Islands. A descriptive account of the families, genera, and species indigenous to Great Britain and Ireland, with notes as to localities, habitats, etc. Vol. II. Staphylinidae. Vol. V: 1-444. L.Reeve and Co., London. http://dx.doi.org/10.5962/bhl.title.48457

Ganglbauer L. 1895. Die Käfer von Mitteleuropa. Die Käfer der österreichisch-ungarischen Monarchie, Deutschlands, der Schweiz, sowie des französischen und italienischen Alpengebietes. Zweiter Band. Familienreiche Staphylinoidea. 1.Theil: Staphylinidae, Pselaphidae: i-vi+1-881. Carl Gerold's Sohn, Wien. http://dx.doi.org/10.5962/bhl.title.8764

Gollkowsky V. 2001. Typenverbleib von Stenus-Arten (Coleoptera, Staphylinidae). Entomologische Blätter 97(2/3): 194.

Goreslavets I.N., Solodovnikov A.Yu., Gildenkov M.Yu. \& Grebennikov K.A. 2002. Staphylinids (Coleoptera, Staphylinidae) of Samara Province: subfamilies Omaliinae, Proteininae, Tachyporinae, Habrocerinae, Oxytelinae, Oxyporinae, Steninae, Euaesthetinae, Paederinae and Staphylininae. [In Russian]. Revue d'Entomologie de la Russie 81(2): 343-355.

Gyllenhal L. 1827. Insecta Suecica descripta. Classis I: Coleoptera sive Eleuterata. Vol.1.ps.IV: i-viii+1-762. Friederichum Fleischer, Lipsiae. http://dx.doi.org/10.5962/bhl.title.8767

Haberman H. 1983. Beitrag zu "Enumeratio Coleopterorum Fennoscandiae et Daniae" (1979) über die Staphyliniden Estlands. Notulae Entomologicae 63: 97-110.

Hamilton J. 1889. Catalogue of the Coleoptera common to North America, Northern Asia and Europe, with the distribution and bibliography. Transactions of the American Entomological Society 16: 88-162. http://dx.doi.org/10.2307/25076523 
Hamilton J. 1894a. Catalogue of the Coleoptera of Alaska, with the synonymy and distribution. Transactions of the American Entomological Society 21: 1-38.

Hamilton J. 1894b. Catalogue of the Coleoptera common to North America, Northern Asia and Europe, with distribution and bibliography. Transactions of the American Entomological Society 21: 345-416.

Hammond P.M. 1972. On the type material of Staphylinidae (Col.) described by T. Marsham and J. F. Stephens. Entomologist's Gazette 23: 129-135.

Hansen V, Hellén W., Jansson A., Munster Th. \& Strand A. 1939. Catalogus Coleopterorum Daniae et Fennoscandiae: i-vii+1-129. Societas pro Fauna et Flora Fennica, Helsingforsiae.

Hardy J. 1851. Family 1. Staphylinidae, Leach. In: Hardy J. \& Bold T.J. A catalogue of the insects of Northumberland and Durham (Part ii). Transactions of the Tyneside Naturalists' Field Club 2: 21-96.

Herman L.H. 2001. Catalog of the Staphylinidae (Insecta: Coleoptera), 1758 to the end of the second millenium. IV. Staphylinine group (part 1). Bulletin of the American Museum of Natural History 265: $1807-2439$.

Heyden L. von 1881. Catalog der Coleopteren von Sibirien mit Einschluss derjenigen der Turanischen Länder, Turkestans und der chinesischen Grenzgebiete. Deutsche Entomologische Zeitschrift, Supplementum: i-xxiv+1-224.

Heyden L. von 1898. Catalog der Coleopteren von Sibirien, mit Einschluss derjenigen des östlichen Caspi-Gebietes, von Turkmenien, Turkestan, Nord-Thibet und des Amur-Gebietes. Nachtrag II: 1-84. A.W.Schade's Buchdruckerei, Berlin.

Horion A. 1963. Faunistik der mitteleuropäischen Käfer. Band IX: Staphylinidae. 1. Teil. Micropeplinae bis Euaestetinae: $\mathrm{i}-\mathrm{xv}+1-335$. Aug. Feyel, Überlingen-Bodensee.

Hochhuth J.H. 1872. Enumeration der in Russischen Gouvernements Kiew und Volhynien bisher aufgefundenen Käfer. [2]. Bulletin de la Société Impériale des Naturalistes de Moscou 44(3-4): 85-177.

Iljin B.S. 1926. Verzeichnis der Käfer des Ekaterinoslavschen Gouvernements. [In Russian]. Revue Russe Entomologique 19(1925) (3-4): 224-228.

Jakobson G.G. 1909. Zhuki Rossii i Zapadnoy Evropy. Fasc. 7. [In Russian]: 481-560. Devrien, St.-Petersburg.

Jakovlev (Jakowlew) A.I. 1902. A list of beetles of Yaroslavl' Government. [In Russian]. Memoires de la Société des Naturalistes de Jaroslaw 1: 88-168.

Johansen J.P. 1914. Danmarks rovbiller eller Billefam. Staphylinide's danske Slcegter og Arter: [1-4]+1-660. Bianco Lunos Bogtrykkeri, København.

Kiesenwetter E.A.H. von 1845. Entomologische Notizen. Entomologische Zeitung, Stettin 6(7): 220-227.

Korge H. 1971. Beiträge zur Kenntnis der Koleopterenfauna Kleinasiens. Annotationes Zoologicae et Botanicae 67: 1-68.

Kraatz G. 1857. [Lieferung 3-4]. In: Naturgeschichte der Insecten Deutschlands. Erste Abteilung. Coleoptera. Zweiter Band: 377-768. Verlag der Nicolaischen Buchhandlung, Berlin.

Kraatz G. 1858. Beitrag zur Käferfauna Griechenlands. Drittes Stück: Staphylinidae (Schlufs), Trichopterygia, Histeridae, Phalacridae, Nitidulariae, Trogositarii, Colydii, Cucujidae, Cryptophagidae, Thorictidae, Mycetophagidae, Dermestini, Byrrhii. Berliner Entomologische Zeitschrift 2: 123-148. 
Krása Th. 1941. Kritische Bemerkungen über europäische, mit Stenus ater nächstverwandte Arten nebst Beschreibung von drei neuen Arten aus dem paläarktischen Gebiet. Časopis Československé Společnosti Entomologické 38: 115-119.

Kuznetsova N.P. 1991. On the fauna of the genus Stenus Latr. (Coleoptera, Staphylinidae) in Belorussia. [In Russian]. In: Fauna and Ecology of Coleoptera of Belorussia: 168-173. Navuka I Tekhnika, Minsk.

Lacordaire J.Th. 1835. Vol.1. [Coleoptera]. In: Boisduval J. \& Lacordaire J.Th. Faune entomologique des environs de Paris; ou species général des insectes qui se trouvent dans un rayon de quinze à vingt lieues aux alentours de Paris: 1-696. Méquignon-Marvis, père et fils, libraires, Paris.

Lebedev A. 1925. Matériaux pour la faune des Coléoptères de la République Tartare. III. [In Russian]. Revue Russe Entomologique 19(1-2): 133-138.

Leinberg A. 1900. Stenus cordaticollis nov. sp. - Meddelanden af Societas pro Fauna et Flora Fennica 26: $187-188$.

Lohse G.A. 1964. Fam. Staphylinidae I (Micropeplinae bis Tachyporinae). In: Freude H., Harde K.W. \& Lohse G.A. (eds.) Die Käfer Mitteleuropas. Band 4: 1-264. Goecke \& Evers, Krefeld.

Lohse G.A. 1985. Betrachtungen über die Gattung Emplenota Casey (Coleoptera, Staphylinidae). Faunistisch-Ökologische Mitteilungen, Kiel 5: 327-330.

Lucas P.H. 1846. Histoire naturelle des animaux articulés. Deuxième partie. Insectes. In: Exploration scientifique de l'Algérie pendant les années 1840, 1841, 1842 publiée par ordre du gouvernement et avec le concours d'une commission académique. Sciences physiques. Zoologie. II: 1-590. Imprimerie Nationale, Paris.

Lucht W.H. 1987. Die Käfer Mitteleuropas. Katalog: 1-342. Goecke \& Evers, Krefeld.

Maeklin F.G. 1853. [Neue Arten]. In: Mannerheim C.G. von. Dritter Nachtrag zur Kaefer-Fauna der Nord-Amerikanischen Laender der Russischen Reiches. Bulletin de la Société Impériale des Naturalistes de Moscou 26(3): 95-273.

Marsham T. 1802. Entomologia britannica, sistens insecta, Britanniae indigena, secundum methodum Linnaeanum disposita. Tomus I. Coleoptera: I-XXXI+1-549. Wilks \& Taylor, Londini.

Monsevičius V. \& Pankevičius R. 2001. Coleoptera species new to Lithuania. Ekologija (Vilnius) 2001(2): 40-45.

Munster T. 1921. Tillaeg til Norges koleopterfauna. Norsk Entomologisk Tidsskrift 1(2): 118-135.

Naomi Sh.-I. \& Puthz V. 1994. Descriptions of Three New Species of the genus Stenus Latreille (Coleoptera, Staphylinidae), with Notes on Synonyms and New Records from Japan. Japanese Journal of Entomology 62(1): 211-221.

Normand H. 1935. Contribution au Catalogue des Coléoptères de Tunisie ( ${ }^{\mathrm{me}}$ fascicule). Bulletin de la Société d'Histoire Naturelle de l'Afrique du Nord 25(9)(1934): 356-390.

Notman H. 1920. Staphylinidae from Florida in the collection of the American Museum of Natural History, with descriptions of new genera and species. Bulletin of the American Museum of Natural History 42: 693-732.

Outerelo R., Palmer M. \& Pons G.X. 1995. Staphylinidae y Pselaphidae (Coleoptera, Staphylinoidea) de s'Albufera de Mallorca (Islas Baleares). Bolletí de la Societat d'Història Natural de les Balears 38: $75-88$.

Palm Th. 1961. Svensk Insektenfauna 9. Skalbaggar. Coleoptera. Kortvingar: Fam. Staphylinidae. Underfam. Oxytelinae, Oxyporinae, Steninae, Euaesthetinae. Häfte 2: 1-126. Stockholm. 
Pisanenko A. \& Puthz V. 1991. Steninen aus Weißrußland (Belorussische SSR) (Coleoptera, Staphylinidae). - Entomologische Blätter für Biologie und Systematik der Käfer 87(3): 165-176.

Poppius R.B. 1899. Förteckning öfver Ryska Karelens Coleoptera. Acta Societatis pro Fauna et Flora Fennica 18(1): 1-125.

Poppius R.B. 1909a. Beiträge zur Kenntnis der Coleopteren-Fauna des Lena-Thales in Ostsibirien, IV. Staphylinidae. Öfversigt af Finska Vetenskaps-Societetens Förhandlingar (A) 51(4): 1-53.

Poppius R.B. 1909b. Die Coleopteren-Fauna der Halbinsel Kanin. Acta Societatis pro Fauna et Flora Fennica 31(8): 1-58.

Puthz V. 1965. Nomenklatorische, systematische u. faunistische Bemerkungen über paläarktische Steninen (Col., Staphylinidae). 6. Beitrag zur Kenntnis der Steninen. Mitteilungen der Deutschen Entomologischen Gesellschaft 24: 25-30.

Puthz V. 1966. Die Stenus-Arten Madeiras und der Kanarischen Inslen (Coleoptera, Staphylinidae). 21. Beitrag zur Kenntnis der Steninen. Entomologische Blätter für Biologie und Systematik der Käfer 62(3): 129-149.

Puthz V. 1967a. Über einige Steninen aus dem Zoologischen Museum Helsinki (Coleoptera. Staphylinidae). 43. Beitrag zur Kenntnis der Steninen. Notulae Entomologicae 47: 47-53.

Puthz V. 1967b. 75. Staphylinidae: Steninae. Ergebnisse der zoologischen Forschungen von Dr. Z.Kaszab in der Mongolei (Coleoptera). 17. Beitrag zur Kenntnis der Steninen. Reichenbachia. Zeitschrift für taxonomische Entomologie des Staatlichen Museums für Tierkunde, Dresden 9(8): 75-83.

Puthz V. 1967c. Catálogo crítico des espécies portuguesas de Stenus (Col. Staphyl.). 31. Beitrag zur Kenntnis der Steninen. Memórias e Estudos do Museu Zoológico da Universidade de Coimbra 299: 1-15.

Puthz V. 1967d. Die Paläarktischen und Afrikanischen Stenus-Typen der Sammlung des Zoologischen Museums Berlin, mit besonderer Berücksichtigung der Erichsonschen Arten. (Coleoptera, Staphylinidae). 18. Beitrag zur Kenntnis der Steninen. Mitteilungen aus dem Zoologischen Museum in Berlin 43(2): 285-309. http://dx.doi.org/10.1002/mmnz.19670430214

Puthz V. 1968. Neue und alte Mittelamerikanische Steninen (Coleoptera. Staphylinidae). 40. Beitrag zur Kenntnis der Steninen. Annotationes Zoologicae et Botanicae 48(1967): 1-13.

Puthz V. 1970a. Einige Bemerkungen zum Catalogus coleopterorum Fennoscandiae et Daniae, Lund 1960, die Gattung Stenus Latr. betreffend (Coleoptera. Staphylinidae). (71. Beitrag zur Kenntnis der Steninen). Notulae Entomologicae 50: 38-40.

Puthz V. 1970b. Über Steninen der Sammlungen des Museo ed Istituto di Zoologia sistematica della Universita' di Torino: Coll. Baudi di Selve und Coll. de Brême (Coleoptera. Staphylinidae). 82. Beitrag zur Kenntnis der Steninen. Bollettino della Società Entomologica Italiana 102(9-10): 166-180.

Puthz V. 1971a. Kritische Faunistik der bisher aus Mitteleuropa bekannten Stenus-Arten nebst systematischen Bemerkungen und Neubeschreibungen (Coleoptera, Staphylinidae). 80. Beitrag zur Kenntnis der Steninen. Entomologische Blätter für Biologie und Systematik der Käfer 67(2): 74-121.

Puthz V. 1971b. Weitere Ergänzungen zum Catalogus Coleopterorum Fennoscandiae et Daniae, Lund 1960, die Gattung Stenus Latr. in Finnland betreffend (Coleoptera, Staphylinidae). (81. Beitrag zur Kenntnis der Steninen). Notulae Entomologicae 51: 33-35.

Puthz V. 1971c. 208. Staphylinidae: Steninae III. Ergebnisse der zoologischen Forschungen von Dr. Z.Kaszab in der Mongolei (Coleoptera). 65. Beitrag zur Kenntnis der Steninen. Faunistische Abhandlungen [Staatliches Museum für Tierkunde, Dresden] 3(13): 135-143. 
Puthz V. 1971d. 104. Beitrag zur Kenntnis der Steninen. Mitteilungen der Deutschen Entomologischen Gesellschaft 30(2): 20-21.

Puthz V. 1972a. Zur Staphylinidenfauna des Balkans: die bisher aus Jugoslawien und angrenzenden Ländern bekannten Steninen (Coleoptera. Staphylinidae). 63.[83!] Beitrag zur Kenntnis der Steninen. Wissenschafliche Mitteilungen des Bosnisch-herzegovinischen Landesmuseums 1(C): 239-292.

Puthz V. 1972b. Zwei neue Stenus-Arten aus der canaliculatus-Gruppe nebst synonymischen Bemerkungen (Coleoptera, Staphylinidae). 102. Beitrag zur Kenntnis der Steninen. Koleopterologische Rundschau 49(1971): 169-175.

Puthz V. 1972c. Some Stenus Latr. from Michigan (Coleoptera: Staphylinidae). 107th contribution to the knowelidge of Steninae. Great Lakes Entomologist 5(1): 11-16.

Puthz V. 1972d. Über einige nordostpaläarktische Stenus-Arten (Coleoptera. Staphylinidae). 121. Beitrag zur Kenntnis der Steninen. Notulae Entomologicae 52: 102-108.

Puthz V. 1972e. A European staphylinid beetle from the Pacific Northwest, new to North America. Journal of the Entomological Society of British Columbia 69: 49.

Puthz V. 1973a. Die von Claudius Rey beschreibenen Stenus-Arten (Coleoptera, Staphylinidae). 120. Beitrag zur Kenntnis der Steninen. Nouvelle Revue d'Entomologie 3(1): 23-31.

Puthz V. 1973b. Revision der nearktischen Steninenfauna (I). Neue nordamericanische Stenus- (s. str. + Nestus) Arten. 133. Beitrag zur Kenntnis der Steninen. Entomologische Blätter für Biologie und Systematik der Käfer 69(3): 189-209.

Puthz V. 1973c. Zur Synonymie und Stellung einiger Stenus-Arten. VI. (Coleoptera. Staphylinidae). 136. Beitrag zur Kenntnis der Steninen. Entomologisk Tidskrift 94(1-2): 48-55.

Puthz V. 1974. Weitere nordostpaläarktische Stenus-Arten (Coleoptera, Staphylinidae). 142. Beitrag zur Kenntnis der Steninen. Notulae Entomologicae 54: 107-113.

Puthz V. 1975a. Stenus von der Anticosti-Insel, Quebec, Canada. Entomologische Blätter für Biologie und Systematik der Käfer 71: 124-125.

Puthz V. 1975b. Stenus caseyi Puthz neu für Canada. Entomologische Blätter für Biologie und Systematik der Käfer 71: 125.

Puthz V. 1987. Ein neuer Stenus (Col. Staphylinidae) aus Japan. 208. Beitrag zur Kenntnis der Steninen. Mitteilungen des Internationalen Entomologischen Vereins, Frankfurt a. M. 11(2/3): 45-48.

Puthz V. 1988. Revision der nearktischen Steninenfauna 3 (Coleoptera, Staphylinidae). Neue Arten und Unterarten aus Nordamerika. Entomologische Blätter für Biologie und Systematik der Käfer 84(3): 132-164.

Puthz V. 1998. Die von Theodor Krása beschriebenen Stenus-Arten (Coleoptera, Staphylinidae) nebst taxonomischen Bemerkungen zu anderen Arten. 257. Beitrag zur Kenntnis der Steninen. Entomologische Blätter für Biologie und Systematik der Käfer 94(3): 147-151.

Puthz V. 2001. Beiträge zur Kenntnis der Steninen. CCLXIX. Zur Ordnung in der Gattung Stenus Latreille, 1796 (Staphylinidae, Coleoptera). Philippia 10(1): 33-42.

Puthz V. 2006. Ein Dutzend neuer paläarktischer Stenus-Arten (Coleoptera, Staphylinidae). 290. Beitrag zur Kenntnis der Steninen. Entomologische Blätter für Biologie und Systematik der Käfer 101(2005) (2-3): 171-196.

Puthz V. 2008. Stenus Latreille und die segenreiche Himmelstochter (Coleoptera, Staphylinidae) Linzer biologische Beiträge 40(1): 137-230. 
Puthz V. \& Zanetti A. 1995. Steninae (generi 074-075). In: Ciceroni A., Puthz V. \& Zanetti A. Fascicolo 48. Coleoptera Polyphaga III (Staphylinidae): 16-19. In: Minelli A., Ruffo S. \& La Posta S. Checklist delle specie della fauna italiana. Edizioni Calderini, Bologna.

Razumovskiy S.M. 1980. Botaniko-geograficheskoye rayonirovaniye Zemli kak predposylka uspeshnoy introduktsii rasteniy. [In Russian]. In: Korovin E.P. (ed.) Introduktsiya tropicheskikh i subtropicheskikh kul'tur: 10-27. Nauka, Moscow.

Renkonen O. 1935. Zwei neue Arten der Gattung Stenus (Col., Staphylinidae) aus Finnland. Annales Entomologici Fennici 1(1): 27-32.

Renkonen O. 1936. Ergänzendes und Berichtigendes über Stenus confusoides Renk. und St. Linnaniemii nom. nov. (= St. distans Renk.; Col. Staphylinidae). Annales Entomologici Fennici 2(4): 178-179.

Renkonen O. 1941. Zerstreute Bemerkungen über Finnische Staphyliniden II. Annales Entomologici Fennici 7(2): 103-109.

Reitter E. 1909. Fauna Germanica. Die Käfer des Deutschen Reiches. Nach der analytischen Methode bearbeitet. II. Band: 1-392. K.G.Lutz' Verlag, Stuttgart.

Rey Cl. 1884. Tribu des Brévipennes. Deuxième groupe: Micropéplides. Troisième groupe: Sténides. Annales de la Société Linnéenne de Lyon (n. ser.) 30: 153-415.

Ryabukhin A.S. 1999. A catalogue of rove beetles (Coleoptera: Staphylinidae exclusive of Aleocharinae) of the northeast of Asia: 1-140. Pensoft, Sofia.

Rye E.C. 1864. Descriptions of the British species of Stenus. [2]. The Entomologist's Monthly Magazine 1: $36-43$.

Rye E.C. 1870. Coleoptera. New British species, corrections of nomenclature, etc., noticed since the publication of the Entomologist's Annual, 1869. The Entomologist's Annual 1870: 31-120.

Ryvkin A.B. 1987. New species of Stenus Latreille 1796 from the Far East of the Soviet Union (Insecta: Coleoptera: Staphylinidae). Senckenbergiana biologica 68(1/3): 149-161.

Ryvkin A.B. 1990. The road-beetle [sic!] subfamily Steninae (Coleoptera, Staphylinidae) in the Caucasus and adjacent areas. [In Russian]. In: Striganova B.R. (ed.) Fauna of Terrestrial Invertebrates of the Caucasus: 137-234. Nauka, Moscow.

Ryvkin A.B. 2011. Contributions to the knowledge of Stenus (Nestus) species of the crassus group (Insecta: Coleoptera: Staphylinidae: Steninae). 1. Four new species from the Russian Far East with taxonomic notes. Baltic Journal of Coleopterology 11(1): 57-72.

Sahlberg C.R. 1832. Insecta Fennica, dissertationibus academicis, A. 1817-1834 editis. Pars I, A: 409-440. Frenckelliana, Helsingforsiae.

Sahlberg J.R. 1871. Anteckningar till Lapplands Coleopter-fauna. Notiser ur Sällskapets pro Fauna et Flora Fennica Förhandlingar. Ny Serie 11: 385-440.

Sahlberg J.R. 1876. Enumeratio Coleopterorum Brachelytrorum Fenniae. Systematisk förteckning öfver de inom Finlands naturalhistoriska område hittils funna Coleoptera Brachelytra jemte uppgift om arternas utbredning och <...>. I. Staphylinidae. Acta Societatis pro Fauna et Flora Fennica 1: 1-248.

Sahlberg J.R. 1880. Bidrag till Nordvestra Sibiriens Insektfauna. Coleoptera. Insamlade under Expeditionerna till Obi och Jenissej 1876 och 1877. I. Cicindelidae, Carabidae, Dytiscidae, Hydrophilidae, Gyrinidae, [...] Staphylinidae och Micropepeplidae. Kongliga Svenska VetenskapsAkademiens Handlingar 17(4): 1-115. 
Sahlberg J.R. 1899. Catalogus praecursorius Coleopterorum in valle fluminis Petschora collectorum. Horae Societatis Entomologicae Rossicae 32: 336-344.

Sahlberg J.R. 1900. Catalogus Coleopterorum faunae Fennicae geographicus. Cum mappis duabus geographicis. Acta Societatis pro Fauna et Flora Fennica 19(4): i-viii+1-132.

Sainte-Claire Deville J. 1906. Catalogue critique des Coléoptères de la Corse. Revue d'Entomologie 25(Suppl. 8): 1-112.

Schaufuss L.W. 1882. Zoologische Ergebnisse von Excursionen auf den Balearen. Verhandlungen der Zoologisch-Botanischen Gesellschaft in Wien 31(1881): 619-624.

Scheerpeltz O. von 1933. Staphylinidae VII (pars 129). In: Schenkling S. (ed.) Coleopterorum Catalogus. Volumen VI. Staphylinidae II: 989-1500. W.Junk, Berlin.

Scheerpeltz O. von 1963. Ergebnisse der von Wilhelm Kühnelt nach Griechenland unternommenen zoologischen Studienreisen. I. (Coleoptera-Staphylinidae). Sitzungsberichte der Österreichische Akademie der Wissenschaften. Mathematisch-naturwissenschaftliche Klasse, Abt. 1 172(9-10): 413-452.

Seidlitz G.K.M. 1872-1875. Fauna Baltica. Die Käfer (Coleoptera) der Ostseeprovinzen Russlands: 1-12 + I-XLII + 1-142 + 1-560. Dorpater Naturforscher-Gesellschaft, Dorpat.

Seidlitz G.K.M. 1887-1891. Fauna Baltica. Die Käfer (Coleoptera) der deutschen Ostseeprovinzen Russlands. Zweite neu bearbeitete Auflage: 1 Taf. + 1-10 + I-LVI + 1-192 + 1-818. Hartungsche Verlagsdruckerei, Königsberg.

Semenov V.B. 2004. Materials on the fauna of the road-beetle [sic!] subfamily Steninae (Coleoptera, Staphylinidae) of the Moscow area. [In Russian]. Bulletin de la Société des Naturalistes de Moscou 109(4): 8-16.

Sharp D. 1887. Staphylinidae. In: Biologia Centrali-Americana. Insecta. Coleoptera. 1(2): 673-824. Taylor \& Francis, London.

Shavrin A.V. 1998. To the knowledge of staphylinid beetle fauna (Coleoptera, Staphylinidae) of the Baikal region. [In Russian]. In: Entomological Problems of Baikalian Siberia. Proceedings of the Regional Conference, December 23-24, 1997: 81-86. Nauka, Novosibirsk.

Shavrin A.V. 2007. The rove beetles (Coleoptera, Staphylinidae) of Pribaikalsky National Park. [In Russian]. Trudy Pribaikal'skogo natsional'nogo parka 2: 141.

Shavrin A.V. \& Puthz V. 2007. Contribution to the Knowledge of the Fauna of Stenus Latreille, 1797 (Coleoptera, Staphylinidae, Steninae) of the Baikal Region. Entomologische Blätter 102(2006)(1-3): 107-136.

Shilov V.F. 1975. Zonal-ecological distribution and zoogeographical analysis of brachelytrate beetles from the subfamily Steninae (Col., Staphylinidae) in the fauna of ASSR Komi. [In Russian]. Vestnik Leningradskogo gosudarstvennogo universiteta 9: 56-60.

Silfverberg H. 1988. Lists of the insect types in the Zoological Museum, University of Helsinki. 8. Coleoptera: Staphylinidae. Acta Entomologica Fennica 52: 15-40.

Smetana A. 1959. Zur Kenntnis der Staphyliniden-Fauna Albaniens (Col., Staphylinidae). Acta Entomologica Musei Nationalis Pragae 33: 195-218.

Smetana A. 2004. Staphylinidae, subfamilies Omaliinae-Dasycerinae, Phloeocharinae-Apateticinae, Piestinae-Staphylininae. In: Löbl I. \& Smetana A. (eds) Catalogue of Palaearctic Coleoptera. Vol. 2. Hydrophiloidea-Histeroidea-Staphylinoidea: 237-272, 329-495, 505-698. Apollo Books, Stenstrup. 
Sokolov A.V. 2003. Rove beetle (Coleoptera, Staphylinidae) fauna in the southern tundra of western Taimyr. [In Russian]. Zoologicheskiy Zhurnal 82(10): 1271-1275.

Solodovnikov A.Yu. 1998. Fauna of staphylinids (Coleoptera, Staphylinidae) of the North-Western Caucasus. Subfamilies Staphylininae, Xantholininae, Paederinae, Steninae, Oxyporinae. [In Russian]. Revue d'Entomologie de la Russie 77(2): 331-354.

Stephens J.F. 1829. A systematic catalogue of British insects: being an attempt to arrange all the hitherto discovered indigenous insects in accordance with their natural affinities. Containing also the references to every English writer on entomology, and to the principal foreign authors. With all the published British genera to the present time: $\mathrm{i}-\mathrm{xxxiv}+1-416+1-388$. Baldwin and Cradock, London. http://dx.doi. org/10.5962/bhl.title.8987

Stephens J.F. 1833. Illustrations of British Entomology; or a synopsis of indigenous insects: containing their generic and specific distinctions; with an account of their metamorphoses, times of appearance, localities, food, and economy, as far as practicable. Mandibulata. Vol. V: 241-304. Baldwin and Cradock, London. http://dx.doi.org/10.5962/bhl.title.8133

Stephens J.F. 1839. A manual of British Coleoptera, or beetles; containing a brief description of all the species of beetles hitherto ascertained to inhabit Great Britain and Ireland; together with a notice of their chief localities, times and places of appearances, etc.: i-xii+1-443. Longman, Orme Brown, Green, and Longmans, London. http://dx.doi.org/10.5962/bhl.title.59227

Strand A. 1954. Coleoptera fra Nordreisa. Norsk Entomologisk Tidsskrift 9(1-2)(1953): 63-71.

Szujecki A. 1958. Zbiór chrząszczy Wojciecha Mączyńskiego. Steninae. (Coleoptera, Staphylinidae). Polskie pismo entomologiczne 27(12): 115-119.

Szujecki A. 1960. O występowaniu w Polsce niektórych gatunków z rodzaju Stenus Latr. (Coleoptera, Staphylinidae). Fragmenta Faunistica 8(19): 293-304.

Szujecki A. 1961. Część XIX. Chrząszcze - Coleoptera. Zeszyt 24 b. Kusakowate - Staphylinidae. Myśliczki - Steninae. Klucze do oznaczania owadów Polski 36: 1-72.

Thomson C.G. 1857. Öfversigt af de arter inom Insektgruppen Stenini, som blifvit funna i Sverige. Öfversigt af Kungliga Svenska Vetenskaps-Akademiens Förhandlingar 14(6): 219-235.

Thomson C.G. 1860. Skandinaviens Coleoptera synoptiskt bearbetade. II. Tom: 1-304. Berlingska Boktryckeriet, Lund.

Tichomirova A.L. 1973. Morfoekologicheskiye osobennosti i filogenez stafilinid (s katalogom fauny SSSR). [In Russian]: 1-191. Nauka, Moscow.

Tichomirova A.L. 1982. Fauna i ekologiya staphylinid (Coleoptera, Staphylinidae) Podmoskov'ya. [In Russian]. In: Pochvennyie bespozvonochnyie Moskovskoy oblasti: 201-222. Nauka, Moscow.

Tottenham C.E. 1937. Note on species of Philonthus in the Stephens collection. The Entomologist's Monthly Magazine 73: 190-191.

Tottenham C.E. 1954. Coleoptera, Staphylinidae. Section (a) Piestinae to Euaesthetinae. Handbooks for the Identification of British Insects 4(8a): 1-79.

Uhlig M. \& Vogel J. 1981. Zur Staphylinidenfauna der Umgebung von Wahren/Müritz (Mecklenburg). - Mitteilungen aus dem Zoologischen Museum in Berlin 57(1): 75-168. http://dx.doi.org/10.1002/ mmnz.19810570107

Waterhouse G.R. 1858. Catalog of British Coleoptera: i-iv+1-117. Taylor and Francis, London. 
Waterhouse G.R. \& Janson E.W. 1855. British species of the genus Stenus. Transactions of the Entomological Society of London (n. ser.) 3: 136-156. http://dx.doi.org/10.1111/j.1365-2311.1855. $\underline{\text { tb02667.x }}$

Weinreich E. 1968. Über den Klebfangapparat der Imagines von Stenus Latr. (Coleopt., Staphylinidae) mit einem Beitrag zur Kenntnis der Jugendstadien dieser Gattung. Zeitschrift für Morphologie der Tiere 62: $162-210$.

Wüsthoff W. 1934. Beitrag zur Kenntnis der mitteleuropäischen Stenusarten. Entomologische Blätter 30(2): 62-64+Taf. I-IV.

Manuscript received: 9 October 2011

Manuscript accepted: 9 December 2011

Published on: 24 May 2012

Topic editor: Malcolm Scoble

In compliance with the $I C Z N$, printed versions of all papers are deposited in the libraries of the institutes that are members of the EJT Consortium: Muséum National d'Histoire Naturelle, Paris, France; National Botanic Garden of Belgium, Meise, Belgium; Royal Museum for Central Africa, Tervuren, Belgium; Natural History Museum, London, United Kingdom; Royal Belgium Institute of Natural Sciences, Brussels, Belgium; Natural History Museum of Denmark, Copenhagen, Denmark. 